\title{
TOPOLOGICAL CYCLIC HOMOLOGY OF SCHEMES
}

\author{
THOMAS GEISSER $^{1} A N D$ LARS HESSELHOLT ${ }^{2}$
}

\section{INTRODUCTION}

In recent years, the topological cyclic homology functor of [4] has been used to study and to calculate higher algebraic $K$-theory. It is known that for finite algebras over the ring of Witt vectors of a perfect field of characteristic $p$, the $p$-adic $K$-theory and topological cyclic homology agree in non-negative degrees, [20]. This has been used to calculate the $p$-adic $K$-theory of truncated polynomial algebras over perfect fields of characteristic $p>0,[21]$, and of rings of integers in unramified extensions of the field $\mathbb{Q}_{p}$ of $p$-adic numbers, [6]. In this paper, we extend the definition of topological cyclic homology to schemes.

The topological Hochschild spectrum $\mathrm{TH}(A)$, recalled in paragraph 2 below, defines, as the ring $A$ varies, a presheaf of spectra on the category of affine schemes. We show in paragraph 3 that the corresponding presheaves of homotopy groups are quasi-coherent sheaves for the étale topology. It follows that the map

$$
\mathrm{TH}(A) \stackrel{\sim}{\longrightarrow} \mathbb{H}^{\cdot}\left((\operatorname{Spec} A)_{\text {ét }}, \mathrm{TH}\right),
$$

where the right hand side is Thomason's hypercohomology construction, is a weak equivalence. The same is true if the étale topology is replaced by any coarser topology $\tau$. This suggests that we define topological Hochschild homology of a scheme $X$ by

$$
\operatorname{TH}\left(X_{\tau}\right)=\mathbb{H}^{\cdot}\left(X_{\tau}, \mathrm{TH}\right)
$$

the choice of topology being irrelevant, at least if $X$ is quasi-compact and quasiseparated. One defines topological cyclic homology from $\mathrm{TH}\left(X_{\tau}\right)$ analogously to the original definition in [4]. In more detail, the circle group acts on $\mathrm{TH}\left(X_{\tau}\right)$ and we write

$$
\operatorname{TR}^{n}\left(X_{\tau} ; p\right)=\operatorname{TH}\left(X_{\tau}\right)^{C_{p^{n-1}}}
$$

for the fixed points by the subgroup $C_{p^{n-1}}$ of the indicated order. There are two maps

$$
R, F: \mathrm{TR}^{n}\left(X_{\tau} ; p\right) \rightarrow \mathrm{TR}^{n-1}\left(X_{\tau} ; p\right),
$$

called the restriction and Frobenius, respectively, and we let $\mathrm{TC}^{n}\left(X_{\tau} ; p\right)$ be the homotopy equalizer. Then

$$
\mathrm{TC}\left(X_{\tau} ; p\right)=\underset{\leftarrow}{\operatorname{holim}} \operatorname{TC}^{n}\left(X_{\tau} ; p\right) .
$$

Again, the choice of topology is irrelevant and the definition agrees with the original definition for affine schemes in that the canonical map

$$
\mathrm{TC}(A ; p) \stackrel{\sim}{\longrightarrow} \mathrm{TC}\left((\operatorname{Spec} A)_{\tau} ; p\right)
$$

\footnotetext{
${ }^{1}$ Supported by Deutsche Forschungsgemeinschaft, ${ }^{2}$ Supported in part by NSF Grant.
} 
is a weak equivalence. The homotopy groups $\pi_{*} \mathrm{TC}(-; p)$ form a pro-presheaf on the category of schemes, and we let $\mathcal{T C}_{i}^{\cdot}$ denote the sheafification for the $\tau$-topology. One then has a descent spectral sequence

$$
E_{2}^{s, t}=H_{\text {cont }}^{s}\left(X_{\tau}, \mathcal{T C}_{-t}^{\cdot}\right) \Rightarrow \mathrm{TC}_{-s-t}(X ; p),
$$

where the left hand side is continuous cohomology in the sense of Jannsen. The spectral sequence usually depends on the choice of the topology $\tau$. Moreover, the cyclotomic trace of [4] extends to the present situation to give a natural map

$$
\operatorname{trc}: K(X) \rightarrow \mathrm{TC}(X ; p)
$$

from the algebraic $K$-theory spectrum of the scheme $X$.

Let $k$ be a perfect field of characteristic $p>0$. In paragraph 4 below, we determine the pro-sheaves $\mathcal{T C}_{i}$ on the category $\mathrm{Sm} / k$ of smooth schemes over $k$ in the étale and Zariski topology. In either topology, the pro-sheaves $\mathcal{T R}_{i}$ are isomorphic to the de Rham-Witt pro-sheaves $W . \Omega^{i}$ by a theorem of the second author, [18, theorem B], and there is an exact sequence of étale pro-sheaves

$$
0 \rightarrow \nu \cdot(i) \rightarrow W \cdot \Omega^{i} \stackrel{1-F}{\longrightarrow} W \cdot \Omega^{i} \rightarrow 0,
$$

where the left hand term is the logarithmic de Rham-Witt pro-sheaves of MilneIllusie. Hence for the étale topology, $\mathcal{T C}_{i}$ and $\nu .(i)$ are isomorphic and the descent spectral sequence takes the form

$$
E_{2}^{s, t}=H_{\text {cont }}^{s}\left(X_{\text {ét }}, \nu \cdot(-t)\right) \Rightarrow \mathrm{TC}_{-s-t}(X ; p) .
$$

We use this to show that $\operatorname{TC}_{i}(X ; p)=0$, when $i>\operatorname{dim} X$, and that $\mathrm{TC}_{i}(X ; p)$ is finite for $i>0$, when $X$ is a smooth projective variety over a finite field.

Let $\epsilon:(\mathrm{Sm} / k)_{\text {ét }} \rightarrow(\mathrm{Sm} / k)_{\text {Zar }}$ be the change of topology map. Since the prosheaf $\nu .(i)$ has only a single non-vanishing higher derived image, one has a short exact sequence of Zariski pro-sheaves

$$
0 \rightarrow R^{1} \epsilon_{*} \nu \cdot(i+1) \rightarrow \mathcal{T} \mathcal{C}_{i} \stackrel{\rho}{\rightarrow} \nu \cdot(i) \rightarrow 0
$$

The cyclotomic trace induces a map of pro-sheaves on $(\mathrm{Sm} / k)_{\text {Zar }}$

$$
\operatorname{trc}:\left(\mathcal{K} / p^{*}\right)_{i} \rightarrow \mathcal{T C}_{i} \text {, }
$$

where the left hand side is $K$-theory with coefficients. Using a theorem of BlochKato, [1, corollary 2.8], and a recent theorem of the first author and M. Levine, [11], we show that the composition

$$
\left(\mathcal{K} / p^{*}\right)_{i} \stackrel{\operatorname{trc}}{\longrightarrow} \mathcal{T C}_{i} \stackrel{\rho}{\rightarrow} \nu \cdot(i)
$$

is an isomorphism of pro-sheaves on $(\mathrm{Sm} / k)_{\text {Zar }}$. Hence for the Zariski topology,

$$
\mathcal{T} \mathcal{C}_{i} \cong \nu \cdot(i) \oplus R^{1} \epsilon_{*} \nu \cdot(i+1)
$$

and the cyclotomic trace corresponds to the inclusion of the first summand. Sheafifying for the étale topology, the map $\rho$ becomes an isomorphism, showing that the cyclotomic trace

$$
\left(\mathcal{K} / p^{*}\right)_{i} \stackrel{\sim}{\longrightarrow} \mathcal{T} \mathcal{C}_{i}
$$

induces an isomorphism of pro-sheaves on $(\mathrm{Sm} / k)_{\text {ét }}$. It follows that for a smooth scheme $X / k$,

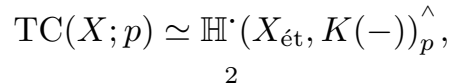


the $p$-completion of the étale hypercohomology spectrum for $K$-theory, and moreover, the cyclotomic trace may be identified with the change of topology map

$$
\mathbb{H}^{\cdot}\left(X_{\text {Zar }}, K(-)\right)_{p}^{\wedge} \rightarrow \mathbb{H}^{\cdot}\left(X_{\text {ét }}, K(-)\right)_{p}^{\wedge}
$$

Indeed, the left hand side is equivalent to $K(X)_{p}^{\wedge}$ by [41, theorem 10.3].

In the case of a field $E$ of characteristic $p>0$, the above equivalence implies that

$$
\left.\mathrm{TC}(E ; p) \simeq \underset{\underset{\alpha}{\operatorname{holim}}}{\longrightarrow} K\left(E_{\alpha}\right)^{h G_{\alpha}}\right)_{p}^{\wedge}
$$

where the homotopy colimit runs over all finite separable extensions $E_{\alpha} / E$ and $G_{\alpha}$ is the Galois group. Let $E^{s}$ be the separable closure of $E$ and let $G_{E}$ be the Galois group. Then on the level of homotopy groups, we show

$$
\mathrm{TC}_{i}(E ; p) \cong K_{i}(E)_{p}^{\wedge} \oplus H_{\text {cont }}^{1}\left(G_{E}, K_{i+1}\left(E^{s}\right)_{p}^{\wedge}\right),
$$

where the second term on right is the continuous cohomology of the profinite group $G_{E}$ acting on the module $K_{i}\left(E^{s}\right)_{p}^{\wedge} \cong K_{i}^{M}\left(E^{s}\right)_{p}^{\wedge}$ in the $p$-adic topology. In the two lowest degrees,

$$
\begin{aligned}
& \operatorname{TC}_{0}(E ; p) \cong \mathbb{Z}_{p} \oplus T_{p} \operatorname{Br}(E) \\
& \operatorname{TC}_{-1}(E ; p) \cong \operatorname{Hom}\left(\operatorname{Gal}\left(E^{s} / E\right), \mathbb{Z}_{p}\right),
\end{aligned}
$$

where $T_{p} \operatorname{Br}(E)$ is the Tate module of the Brauer group. Let us also note that for a field of characteristic different from $p, \mathrm{TC}_{*}(E ; p) \cong \Omega_{E / \mathbb{Q}}^{*}$. As an example, we calculate the topological cyclic homology of a field complete under a discrete valuation with perfect residue field.

In paragraph 5, we examine the Zariski descent sequence

$$
E_{2}^{s, t}=H^{s}\left(X_{\text {Zar }}, \mathcal{T C}_{-t}^{\cdot}\right) \Rightarrow \mathrm{TC}_{-s-t}(X ; p)
$$

for $X$ a smooth scheme over $k$.. The $E_{2}$-term decomposes according to the decomposition of the Zariski pro-sheaf $\mathcal{T C}_{-t}$ stated earlier, but the differentials usually mix the two summands. Indeed, we show that when $X$ is affine, the composite

$$
H_{\text {cont }}^{s}\left(X_{\text {Zar }}, R^{1} \epsilon_{*} \nu \cdot(-t+1)\right) \longmapsto E_{2}^{s, t} \stackrel{d_{2}}{\longrightarrow} E_{2}^{s+2, t-1} \rightarrow H^{s+2}\left(X_{\text {Zar }}, \nu \cdot(-t+1)\right)
$$

has the same kernel and image as the $d_{2}$-differential in the change of topology spectral sequence sequence for sheaf cohomology,

$$
E_{2}^{i, j}=H_{\text {cont }}^{i}\left(X_{\text {Zar }}, R^{j} \epsilon_{*} \nu \cdot(-t+1)\right) \Rightarrow H_{\text {cont }}^{i+j}\left(X_{\text {ét }}, \nu \cdot(-t+1)\right) .
$$

It follows that the descent sequence collapses at $E_{3}$ with the all non-zero groups concentrated on the lines $s=0,1$. This in turn identifies the kernel of the cyclotomic trace

$$
\operatorname{trc}: K_{i}\left(X ; \mathbb{Z}_{p}\right) \rightarrow \mathrm{TC}_{i}(X ; p)
$$

with the second step of the filtration of the left hand group induced by the Zariski descent sequence, $F^{2} K_{i}\left(X, \mathbb{Z}_{p}\right)$. In particular, the part of $K$-theory of weight greater than $i+1$ cannot be detected by topological cyclic homology.

Finally, we mention two results used in the proofs. We show in the appendix that for a commutative ring $A$, the cyclotomic trace map is a map of symmetric ring spectra. In particular, the induced map of homotopy groups

$$
\operatorname{trc}_{*}: K_{*}(A) \rightarrow \mathrm{TC}_{*}(A ; p)
$$


is a ring homomorphism. Moreover, we give a description of the restriction of the trace to the subgroup of units $A^{\times} \subset K_{1}(A)$ in terms of the Teichmüller section and Connes' $B$-operator. See lemma 4.2 .3 below for a precise statement.

In this paper, pro-sheaves of abelian groups on a site will alway be considered modulo the Serre subcategory of Mittag-Leffler zero pro-sheaves, i. e. a sequence of pro-sheaves will be called exact if its cohomology pro-sheaves are Mittag-Leffler zero. We recall that short exact sequences of pro-sheaves induce long-exact sequences on continuous cohomology in the sense of Jannsen, [25]. Moreover, spectra will mean symmetric spectra in the sense of Hovey-Shipley-Smith, [22].

\section{TOpological CYCliC HOMOLOGY}

2.1. It will be most convenient for our purpose here to work in the closed model category of symmetric spectra recently constructed by Hovey-Shipley-Smith, [22]. The notion of a symmetric spectrum is close to Bökstedt's notion of a functor with stabilization, [3]. We recall the definition.

A symmetric spectrum $E$ consists of a sequence of pointed spaces $E_{n}, n \geq 0$, together with, for every $n \geq 0$, an action by the symmetric group $\Sigma_{n}$ on $E_{n}$, and for every pair $m, n \geq 0$, pointed maps

$$
\lambda_{m, n}: S^{m} \wedge E_{n} \rightarrow E_{m+n},
$$

which are $\Sigma_{m} \times \Sigma_{n}$-equivariant, when $\Sigma_{m} \times \Sigma_{n}$ acts via the inclusion $\Sigma_{m} \times \Sigma_{n} \subset$ $\Sigma_{m+n}$ on the right. Here and throughout, $S^{m}$ is the $m$-fold smash product of the circle $\Delta^{1} / \partial \Delta^{1}$. Let $\sigma_{n, m} \in \Sigma_{m+n}$ be the permutation $i \mapsto i+n(\bmod m+n)$ and define

$$
\rho_{m, n}: E_{m} \wedge S^{n} \rightarrow E_{m+n}
$$

as the composite $\sigma_{n, m} \circ \lambda_{n, m} \circ \mathrm{tw}_{E_{m}, S^{n}}$, where $\mathrm{tw}_{E_{m}, S^{n}}$ permutes the smash factors. Then we require that, for every $n \geq 0$,

$$
\lambda_{0, n}=E_{n},
$$

and for every triple $k, m, n \geq 0$,

$$
\lambda_{k, m+n} \circ\left(S^{k} \wedge \lambda_{m, n}\right)=\lambda_{k+m, n}
$$

and

$$
\rho_{k+m, n} \circ\left(\lambda_{k, m} \wedge S^{n}\right)=\lambda_{k, m+n}\left(S^{k} \wedge \rho_{m, n}\right)
$$

up to coherent isomorphisms. A map of symmetric spectra is a sequence of maps $f_{n}: E_{n} \rightarrow F_{n}$ of pointed spaces which commutes with all the structure.

We say that a symmetric spectrum $E$ is quasi-fibrant if for all $m, n \geq 1$, the adjoint map of $\lambda_{m, n}$ is a weak equivalence

$$
E_{n} \stackrel{\sim}{\longrightarrow} F\left(S^{m}, E_{m+n}\right) .
$$

For a symmetric spectrum of pointed simplicial sets, we require in addition that $E_{n}$ be fibrant as a simplicial set, for all $n \geq 1$. The homotopy groups of a symmetric spectrum are defined by

$$
\pi_{n} E=\underset{m}{\underset{4}{\lim _{m}}} \pi_{m+n} E_{m}
$$


where the maps in the limit system are induced from the maps $\lambda_{1, n}$. We say that $E$ is convergent if for all $n$, this limit is attained. A convergent symmetric spectrum is connective if the negative homotopy groups vanish. A map $f: E \rightarrow F$ of quasi-fibrant symmetric spectra is a weak equivalence if and only if it induces an isomorphism on homotopy groups.

The categorical limit and colimit of a diagram of symmetric spectra is equal to the limit and colimit formed at each level. Homotopy limits and homotopy colimits are formed levelwise. Limits and homotopy limits preserve quasi-fibrant symmetric spectra, but this is not the case of colimits and homotopy colimits in general.

A symmetric ring spectrum is a symmetric spectrum $E$ together with, for every $n \geq 0$, a $\Sigma_{n}$-equivariant map

$$
\mathbf{1}_{n}: S^{n} \rightarrow E_{n},
$$

and for every pair $m, n \geq 0$, a $\Sigma_{m} \times \Sigma_{n}$-equivariant map

$$
\mu_{m, n}: E_{m} \wedge E_{n} \rightarrow E_{m+n}
$$

such that, for every pair $m, n \geq 0$,

$$
\mu_{m, n} \circ\left(\mathbf{1}_{m} \wedge E_{n}\right)=\lambda_{m, n}, \quad \mu_{m, n} \circ\left(E_{m} \wedge \mathbf{1}_{n}\right)=\rho_{m, n},
$$

and for every triple $k, m, n$,

$$
\mu_{k+m, n} \circ\left(\mu_{k, m} \wedge E_{n}\right)=\mu_{k, m+n} \circ\left(E_{k} \wedge \mu_{m, n}\right) .
$$

We note that it follows from these axioms that $\mathbf{1}_{m+n}=\mu_{m, n} \circ\left(\mathbf{1}_{m} \wedge \mathbf{1}_{n}\right)$. Finally, if in addition

$$
\mu_{n, m} \circ \mathrm{tw}_{E_{m}, E_{n}}=\sigma_{m, n} \circ \mu_{m, n}
$$

then we say that $E$ is commutative. A map of symmetric ring spectra is a sequence of maps $f_{n}: E_{n} \rightarrow F_{n}$ which commutes with all the structure.

We define the Eilenberg-MacLane spectrum $\tilde{A}$ associated with the ring $A$ as follows: If $X$ is a pointed set we write $A(X)$ for the quotient of the free $A$-module generated by $X$ by the submodule generated by the basepoint. We define

$$
\tilde{A}_{n}=\left|A\left(S_{.}^{n}\right)\right|
$$

with the action by the symmetric group $\Sigma_{n}$ induced from the permutation of the $n$ smash factors in $S^{n}$. The multiplication in $A$ induces a $\Sigma_{m} \times \Sigma_{n}$-equivariant map

$$
\mu_{m, n}: \tilde{A}_{m} \wedge \tilde{A}_{n} \rightarrow \tilde{A}_{m+n}
$$

and the assignment $x \mapsto 1 \cdot x$ defines a $\Sigma_{n}$-equivariant map

$$
\mathbf{1}_{n}: S^{n} \rightarrow \tilde{A}_{n} .
$$

If is easily verified that $\tilde{A}$ is a symmetric spectrum, commutative if $A$ is.

2.2. We next recall the definition of Bökstedt's topological Hochschild homology of a symmetric ring spectrum $E$. We will use the model from $[20,2.7]$, which gives us a quasi-fibrant symmetric spectrum $\mathrm{TH}(E)$ with a continuous $S^{1}$-action.

Let $E$ be a symmetric ring spectrum and let $X$ be a pointed space. We begin by defining a cyclic space

$$
[r] \mapsto \operatorname{THH}(E ; X)[r]
$$


The geometric realization, which we denote by $\operatorname{THH}(E ; X)$, will then be an $S^{1}$ space. Let $I$ be the category with objects the finite sets

$$
\underline{i}=\{1,2, \ldots, i\}, \quad i \geq 1,
$$

and the empty set $\underline{0}$ and morphisms all injective maps.

We define a functor $G_{0}(E ; X): I \rightarrow \mathcal{T}_{*}$ from $I$ to the category of pointed spaces. It is given on objects by

$$
G_{0}(E ; X)(\underline{i})=F\left(S^{i}, X \wedge E_{i}\right),
$$

the pointed mapping space, and on the standard inclusion $\iota: \underline{i} \rightarrow \underline{j}$ by the map $G_{0}(E ; X)(\iota)$, which takes $f: S^{i} \rightarrow X \wedge E_{i}$ to the composite

$$
S^{i} \wedge S^{j-i} \stackrel{f \wedge \mathbf{1}_{j-i}}{\longrightarrow} X \wedge E_{i} \wedge E_{j-i} \stackrel{X \wedge \mu_{i, j-i}}{\longrightarrow} X \wedge E_{j} .
$$

The symmetric group $\Sigma_{i}$ acts on on $S^{i}$ and $E_{i}$ and on the mapping space $G_{0}(E ; X)(\underline{i})$ by conjugation. This specifies the functor $G_{0}(E ; X)$ on automorphisms, and finally, every map in $I$ may be written as the standard inclusion followed by an automorphism.

More generally, we have functors

$$
G_{r}(E ; X): I^{r+1} \rightarrow \mathcal{T}_{*}
$$

defined on objects by

$$
G_{r}(E ; X)\left(\underline{i}_{0}, \ldots, \underline{i}_{r}\right)=F\left(S^{i_{0}} \wedge \cdots \wedge S^{i_{r}}, X \wedge E_{i_{0}} \wedge E_{i_{1}} \wedge \cdots \wedge E_{i_{r}}\right),
$$

and whose value on morphisms is defined component-wise. Then the space of $r$ simplices is

$$
\operatorname{THH}(E ; X)[r]=\underset{I^{r+1}}{\operatorname{holim}} G_{r}(E ; X) .
$$

We recall the definition of the face maps in $\operatorname{THH}(E ; X)$ in detail, the remaining cyclic structure maps being defined analogously. The category $I$ has a monoidal structure given by concatenation of sets and maps,

$$
\sqcup: I \times I \rightarrow I,
$$

but note that $(I, \sqcup)$ is not symmetric monoidal. Let $\mathfrak{d}_{j}: I^{r+1} \rightarrow I^{r}, 0 \leq j \leq r$, be the functors defined by

$$
\begin{aligned}
\mathfrak{d}_{j}\left(\underline{i}_{0}, \ldots, \underline{i}_{r}\right) & =\left(\underline{i}_{0}, \ldots, \underline{i}_{j} \sqcup \underline{i}_{j+1}, \ldots, \underline{i}_{r}\right), & 0 \leq j<r, \\
& =\left(\underline{i}_{r} \sqcup \underline{i}_{0}, \underline{i}_{1}, \ldots, \underline{i}_{r-1}\right), & j=r,
\end{aligned}
$$

on objects and similar on morphisms, and let

$$
\delta_{j}: G_{r}(E ; X) \rightarrow G_{r-1}(E ; X) \circ \mathfrak{d}_{j}, \quad 0 \leq j \leq r,
$$

be the natural transformations defined by

$$
\begin{array}{rlrl}
\delta_{j} & =F\left(S^{i_{0}} \wedge \ldots \wedge S^{i_{r}}, X \wedge E_{i_{0}} \wedge \ldots \wedge \mu_{i_{j}, i_{j+1}} \wedge \ldots \wedge E_{i_{r}}\right), & 0 \leq j<r, \\
& =F\left(S^{i_{r}} \wedge S^{i_{0}} \wedge \ldots \wedge S^{i_{r-1}}, X \wedge \mu_{i_{r}, i_{0}} \wedge E_{i_{1}} \wedge \ldots \wedge E_{i_{r-1}}\right) \circ \tau^{\#}, \quad j=r,
\end{array}
$$

where $\tau^{\#}=F\left(\tau^{-1}, \tau\right)$ and $\tau$ permutes the smash factors as indicated. Then the face maps

$$
d_{j}: \operatorname{THH}(E ; X)[r] \rightarrow \operatorname{THH}(E ; X)[r-1] \quad, 0 \leq j \leq r,
$$


are given by the composites

$$
\underset{I^{r+1}}{\operatorname{holim}} G_{r}(E ; X) \stackrel{\delta_{j}}{\longrightarrow} \underset{I^{r+1}}{\operatorname{holim}} G_{r-1}(E ; X) \circ \mathfrak{d}_{j} \underset{\overrightarrow{I^{r}}}{\stackrel{\text { can }}{\longrightarrow}} \underset{\operatorname{holim}}{\longrightarrow} G_{r-1}(E ; X),
$$

where can is the canonical map of homotopy colimits, cf. [7].

In order to define the symmetric spectrum $\mathrm{TH}(E)$, we modify the construction above as follows: Let $P$ be a finite ordered set and let $I^{P}$ be the product, indexed by $P$, of copies of $I$. Iterated concatenation according to the ordering of $P$ specifies a functor

$$
\sqcup_{P}: I^{P} \rightarrow I .
$$

Let $G_{r}^{P}(E ; X)$ be the functor obtained by composing $G_{r}(E ; X)$ with $\sqcup_{P}$ in each factor and note that component wise concatenation gives a functor

$$
\sqcup^{P}: I^{P} \times I^{P} \rightarrow I^{P} .
$$

We then define the $S^{1}$-space $\mathrm{THH}^{P}(E ; X)$ as the geometric realization of the cyclic space with $r$-simplices

$$
\operatorname{THH}^{P}(E ; X)[r]=\underset{\left(I^{P}\right)^{r+1}}{\operatorname{holim}} G_{r}^{P}(E ; X)
$$

and cyclic structure maps defined as in (3), but with the functor $\sqcup^{P}$ in place of $\sqcup$. Let $P \rightarrow P^{\prime}$ be an injective, but not necessarily order preserving map. Extending by zeros, we get a functor $I^{P} \rightarrow I^{P^{\prime}}$ and in turn an $S^{1}$-equivariant map

$$
\iota: \operatorname{THH}^{P}(E ; X) \rightarrow \mathrm{THH}^{P^{\prime}}(E ; X)
$$

which makes $\operatorname{THH}^{P}(E ; X)$ functorial in the variable $P$. In particular, the symmetric group $\Sigma_{P}$ acts on $\operatorname{THH}^{P}(E ; X)$.

Let $Q$ be a finite ordered set and let $S^{Q}$ be the $|Q|$-sphere with the $\Sigma_{Q}$-action given by permuting the smash factors. By naturality, we get an induced $\Sigma_{Q}$-action on $\operatorname{THH}^{P}\left(E ; S^{Q}\right)$. Moreover, there is a natural $\Sigma_{Q^{\prime}} \times \Sigma_{Q} \times S^{1}$-equivariant map

$$
l: S^{Q^{\prime}} \wedge \mathrm{THH}^{P}\left(E ; S^{Q}\right) \rightarrow \mathrm{THH}^{P}\left(E ; S^{Q^{\prime} \sqcup Q}\right)
$$

which is functorial in $P$. We let both $P$ and $Q$ be equal to $\mathbf{n}$ and define the $n$-th space of the topological Hochschild spectrum by

$$
\mathrm{TH}(E)_{n}=\mathrm{THH}^{P}\left(E ; S^{Q}\right),
$$

with $\Sigma_{n}$ acting via the diagonal embedding $\Sigma_{n} \rightarrow \Sigma_{P} \times \Sigma_{Q}$. The map

$$
\lambda_{m, n}: S^{m} \wedge \mathrm{TH}(E)_{n} \rightarrow \mathrm{TH}(E)_{m+n}
$$

is defined as the $\Sigma_{m} \times \Sigma_{n} \times S^{1}$-equivariant map defined as the composite

$$
S^{m} \wedge \mathrm{THH}^{Q}\left(E ; S^{Q}\right) \stackrel{S^{m} \wedge \iota}{\longrightarrow} S^{m} \wedge \mathrm{THH}^{Q^{\prime} \sqcup Q}\left(E ; S^{Q}\right) \stackrel{l}{\longrightarrow} \mathrm{THH}^{Q^{\prime} \sqcup Q}\left(E ; S^{Q^{\prime} \sqcup Q}\right),
$$

where $Q^{\prime}=\underline{m}$ and $Q=\underline{n}$. We recall from [20, proposition 2.4] that

Proposition 2.2.1. Let $E$ be a convergent connective symmetric ring spectrum. Then with these definitions, $\mathrm{TH}(E)$ is a quasi-fibrant symmetric spectrum with an $S^{1}$-action. Moreover, for every finite subgroup $C \subset S^{1}$, the fixed set $\operatorname{TH}(E)^{C}$ is a quasi-fibrant symmetric spectrum. 
If $E$ is a commutative symmetric spectrum, then $\mathrm{TH}(E)$ is again a commutative symmetric ring spectrum, $[20,2.7 .1]$. We consider products in the appendix. We also note that the zeroth space

$$
\mathrm{TH}(E)_{0}=\left|N^{\mathrm{cy}}\left(E_{0}\right)\right|
$$

is equal to the realization of the cyclic bar-construction on the pointed monoid $E_{0}$ with multiplication given by $\mu_{0,0}$.

2.3. The basis of the definition of the topological cyclic homology of [4] is the existence of a special family of maps, the restriction maps. These are maps of symmetric spectra

$$
R_{r}: \mathrm{TH}(E)^{C_{r s}} \rightarrow \mathrm{TH}(E)^{C_{s}},
$$

whose definition we now recall. We first remind the reader of the edgewise subdivision of $[4, \S 1]$.

Let $Y: \Delta^{\mathrm{op}} \rightarrow \mathcal{C}$ be a simplicial object in the category $\mathcal{C}$. The $r$-fold concatenation of sets and maps defines a functor $\operatorname{sd}_{r}: \Delta \rightarrow \boldsymbol{\Delta}$ and the $r$-fold subdivision of $Y$ is defined as the composite

$$
\operatorname{sd}_{r} Y: \boldsymbol{\Delta}^{\mathrm{op}} \stackrel{\mathrm{sd}_{r}^{\mathrm{op}}}{\longrightarrow} \boldsymbol{\Delta}^{\mathrm{op}} \stackrel{Y}{\longrightarrow} \mathcal{C} .
$$

In particular, the $n$-simplices are $\left(\operatorname{sd}_{r} Y\right)[n]=Y[r(n+1)-1]$. When $\mathcal{C}$ is the category of sets, there is a canonical (non-simplicial) homeomorphism

$$
D_{r}:\left|\operatorname{sd}_{r} Y\right| \rightarrow|Y|
$$

induced from

$$
\text { id } \times d_{r}: Y[r n-1] \times \Delta^{n-1} \rightarrow Y[r n-1] \times \Delta^{r n-1},
$$

where $d_{r}: \Delta^{n-1} \rightarrow \Delta^{r n-1}$ is the diagonal embedding.

Suppose that $X: \boldsymbol{\Lambda} \rightarrow \mathcal{C}$ is a cyclic object. The cyclic operator $t_{n}: X[n] \rightarrow X[n]$ satisfies $t_{n}^{n+1}=\mathrm{id}$, so we get an action by the cyclic group $C_{r}$ on $\left(\operatorname{sd}_{r} X\right)[n]$ by letting the generator act as $t_{r(n+1)-1}^{n+1}$. One easily checks that the action is through simplicial maps such that $\operatorname{sd}_{r} X$ is a simplicial $C_{r}$-object. If $\mathcal{C}$ has finite limits, we can take fixed points $\left(\operatorname{sd}_{r} X\right)^{C_{r}}$. It turns out that this is again a cyclic object with the cyclic operator on $\left(\operatorname{sd}_{r} X\right)^{C_{r}}[n]$ given by $t_{r(n+1)-1}$. In the case of cyclic sets, it is proved in $[4, \S 1]$ that the homeomorphism $D_{r}$ induces an $S^{1}$-equivariant homeomorphism

$$
D_{r}:\left|\left(\operatorname{sd}_{r} X\right)^{C_{r}}\right| \rightarrow \rho_{r}^{*}|X|^{C_{r}},
$$

where the circle acts through the root isomorphism $\rho_{r}: S^{1} \rightarrow S^{1} / C_{r}, \rho_{r}(z)=$ $z^{1 / r} C_{r}$, on the right.

We now construct the maps (5). We consider only the case $s=1$, the general case being similar. The cyclic space

$$
\operatorname{sd}_{r}\left(\operatorname{THH}^{\mathbf{n}}\left(E ; S^{n}\right)[-]\right)^{C_{r}}
$$

has $k$-simplices

$$
\left.\underset{\left(I^{\mathbf{n}}\right)^{r(k+1)}}{\underset{8}{\operatorname{holim}}} G_{r(k+1)-1}^{\mathbf{n}}\left(E ; S^{n}\right)\right)^{C_{r}},
$$


and the diagonal functor

$$
\Delta_{r}:\left(I^{\mathbf{n}}\right)^{k+1} \stackrel{\Delta_{r}}{\longrightarrow}\left(I^{\mathbf{n}}\right)^{r(k+1)}, \quad\left(\mathbf{i}_{0}, \ldots, \mathbf{i}_{k}\right) \mapsto\left(\mathbf{i}_{0}, \ldots, \mathbf{i}_{k}, \ldots, \mathbf{i}_{0}, \ldots, \mathbf{i}_{k}\right)
$$

induces a homeomorphism

$$
\left.\underset{\left(I^{\mathbf{n}}\right)^{k}+1}{\operatorname{holim}}\left(G_{r(k+1)-1}^{\mathbf{n}}\left(E ; S^{n}\right) \circ \Delta_{r}\right)^{C_{r}} \stackrel{\cong}{\cong} \underset{\left(I^{\mathbf{n}}\right)^{\overrightarrow{r(k+1)}}}{\underset{h o l i m}{r}} G_{r(k+1)-1}^{\mathbf{n}}\left(E ; S^{n}\right)\right)^{C_{r}} .
$$

Indeed, this may be seen by considering the simplicial replacement of the homotopy colimits, $[7, \S \mathrm{XII}]$. The left hand side is

$$
\underset{\left(I^{\left(\mathbf{n}^{k}\right.}\right)^{k}}{\operatorname{holim}} F\left(\left(S^{\sqcup_{\mathbf{n}} \mathbf{i}_{0}} \wedge \cdots \wedge S^{\sqcup_{\mathbf{n}} \mathbf{i}_{k}}\right)^{\wedge r},\left(E_{\sqcup_{\mathbf{n}} \mathbf{i}_{0}} \wedge \cdots \wedge E_{\sqcup_{\mathbf{n}} \mathbf{i}_{k}}\right)^{\wedge r}\right)^{C_{r}},
$$

where $C_{r}$ acts by cyclically permuting the smash factors in each variable and by conjugation on the mapping space: the fixed set is the space of equivariant maps. Restricting an equivariant map to the fixed set defines a map

$$
\begin{aligned}
& \underset{\left(I^{\mathbf{n}}\right)^{k+1}}{\operatorname{holim}} F\left(\left(S^{\sqcup_{\mathbf{n}} \mathbf{i}_{0}} \wedge \cdots \wedge S^{\sqcup_{\mathbf{n}} \mathbf{i}_{k}}\right)^{\wedge r},\left(E_{\sqcup_{\mathbf{n}} \mathbf{i}_{0}} \wedge \cdots \wedge E_{\sqcup_{\mathbf{n}} \mathbf{i}_{k}}\right)^{\wedge r}\right)^{C_{r}} \\
& \stackrel{\text { res }}{\longrightarrow} \underset{\left(I^{\mathbf{n}}\right)^{k+1}}{\operatorname{holim}} F\left(S^{\sqcup_{\mathbf{n}} \mathbf{i}_{0}} \wedge \cdots \wedge S^{\sqcup_{\mathbf{n}} \mathbf{i}_{k}}, E_{\sqcup_{\mathbf{n}} \mathbf{i}_{0}} \wedge \cdots \wedge E_{\sqcup_{\mathbf{n}} \mathbf{i}_{k}}\right),
\end{aligned}
$$

and one checks that as $k$ varies, this induces a map of cyclic spaces

$$
\operatorname{sd}_{r}\left(\operatorname{THH}^{\mathbf{n}}\left(E ; S^{n}\right)[-]\right)^{C_{r}} \rightarrow \operatorname{THH}^{\mathbf{n}}\left(E ; S^{n}\right)[-] .
$$

Realizing and precomposing with the inverse of the map (6), we get the desired map (5) of symmetric spectra.

The Frobenius maps

$$
F_{r}: \mathrm{TH}(E)^{C_{r s}} \rightarrow \mathrm{TH}(E)^{C_{s}}
$$

are defined as the obvious inclusion. We fix a prime $p$ and write $R$ and $F$ for $R_{p}$ and $F_{p}$, respectively. Then one defines

$$
\begin{aligned}
& \operatorname{TR}^{n}(E ; p)=\operatorname{TH}(E)^{C_{p^{n-1}}} \\
& \operatorname{TC}^{n}(E ; p)=\underset{\longleftarrow}{\operatorname{holim}}\left(\operatorname{TR}^{n}(E ; p) \underset{F}{\stackrel{R}{\rightleftarrows}} \operatorname{TR}^{n-1}(E ; p)\right),
\end{aligned}
$$

the homotopy equalizer of $R$ and $F$. As $n$ varies, these constructions form prosystems $\mathrm{TR}^{*}(E ; p)$ and $\mathrm{TC}^{\cdot}(E ; p)$ of quasi-fibrant symmetric spectra, and then

$$
\begin{aligned}
& \mathrm{TR}(E ; p)=\underset{R}{\operatorname{holim}} \operatorname{TR}^{n}(E ; p) \\
& \mathrm{TC}(E ; p)=\underset{\longleftarrow}{\operatorname{holim}} \mathrm{TC}^{n}(E ; p) .
\end{aligned}
$$

Since homotopy limits commute, the diagram

$$
\mathrm{TC}(E ; p) \longrightarrow \mathrm{TR}(E ; p) \underset{F}{\stackrel{R}{\longrightarrow}} \mathrm{TR}(E ; p)
$$

is a homotopy equalizer.

Finally, we recall the homotopy orbit spectrum $E_{h G}$ of a finite group $G$ acting on a symmetric spectrum $E$ which is defined as follows: We may view $E$ as functor 
$\underline{E}$ from the category $\mathcal{G}$ with one object with endomorphisms $G$ to the category of symmetric spectra and form the homotopy colimit

$$
\underset{\mathcal{G}}{\operatorname{holim}} \underline{E} \text {. }
$$

However, this is only fibrant in trivial cases, so we define $E_{h G}$ to be the fibrant replacement. There is a strongly convergent first quadrant spectral sequence

$$
E_{s, t}^{2}=H_{s}\left(G ; \pi_{t} E\right) \Rightarrow \pi_{s+t} E_{h G}
$$

from the group homology of the $G$-module $\pi_{*} E$. The following result is instrumental in the analysis of topological cyclic homology. It was proved in $[5, \S 1]$, but see also $[20, \S 2]$.

Theorem 2.3.1. There is a cofibration sequence of quasi fibrant symmetric spectra

$$
\mathrm{TH}(E)_{h C_{p^{n}}} \stackrel{N}{\longrightarrow} \mathrm{TR}^{n+1}(E ; p) \stackrel{R}{\longrightarrow} \mathrm{TR}^{n}(E ; p),
$$

where the map $N$ is the norm map or hypertransfer.

We thank Stefan Schwede for help with the argument below.

Corollary 2.3.2. Suppose the ring $A$ is the filtered colimit of rings $A_{\alpha}$. Then the canonical map

$$
\underset{\alpha}{\lim _{*}} \pi_{*} \mathrm{TC}^{n}\left(\tilde{A}_{\alpha} ; p\right) \stackrel{\sim}{\longrightarrow} \pi_{*} \operatorname{TC}^{n}(\tilde{A} ; p)
$$

is an isomorphism.

Proof. We recall that a filtered colimit is an exact functor. It is therefore sufficient to show that

$$
\underset{\alpha}{\lim } \pi_{*} \mathrm{TH}(\tilde{A}) \stackrel{\sim}{\longrightarrow} \pi_{*} \mathrm{TH}(\tilde{A})
$$

is an isomorphism. Indeed, the spectral sequence (9) then shows that the same is true for the homotopy orbits $\pi_{*} \mathrm{TH}(-)_{h C_{p^{n}}}$ and a five-lemma argument based on 2.3.1 implies the corresponding isomorphism for $\pi_{*} \mathrm{TR}^{n}(-; p)$. The statement then follows from a five-lemma argument based on (8).

In order to prove the above isomorphism, we encounter the following technical difficulty. As is well-known, a map from a compact space to a countable filtered colimit of spaces along closed inclusions factors through some stage. However, this is false for filtered colimits in general as one sees by considering the union of the countable subsets of the unit interval. This example is due to Gaunce Lewis. We overcome this difficulty by replacing $\mathrm{TH}(\tilde{A})$ by a different model. Recall that for every pair $X$. and $Y$. of pointed simplicial sets there are natural weak equivalences

$$
F(|X .|,|Y .|) \stackrel{\sim}{\longrightarrow} F\left(|X .|,\left|\operatorname{Ex}^{\infty}(Y .)\right|\right) \stackrel{\sim}{\longleftarrow}\left|F\left(X ., \operatorname{Ex}^{\infty}(Y .)\right)\right|,
$$

where $\mathrm{Ex}^{\infty}$ is Kan's functor, [27]. Moreover, the right hand side is the realization of a fibrant simplicial set, so the homotopy groups may be calculated on the simplicial level. The advantage of this is that if $X$. is finite and if $Y$. is the filtered colimit of pointed simplicial sets $Y_{\alpha}$, then the canonical map

$$
\underset{\alpha}{\lim _{\alpha}} F\left(X ., \operatorname{Ex}^{\infty}\left(Y_{\alpha, \cdot}\right)\right) \rightarrow F\left(X ., \operatorname{Ex}^{\infty}(Y .)\right)
$$


is an isomorphism of fibrant simplicial sets. Indeed, every map from a finite simplicial set to a filtered colimit of simplicial sets factors through some stage of the limit. We now define a quasi-fibrant symmetric spectrum $\mathrm{TH}^{\prime}(\tilde{A})$ of simplicial sets. The definition is analogous to the definition of $\mathrm{TH}(\tilde{A})$ with the following exceptions: We replace the functor (2) by the functor

$$
G_{r}^{\prime}(\tilde{A} ; X)\left(\underline{i}_{0}, \ldots, \underline{i}_{r}\right)=F\left(S_{.}^{i_{0}} \wedge \cdots \wedge S_{._{r}}^{i_{r}}, \operatorname{Ex}^{\infty}\left(X \wedge A_{\alpha}\left(S_{.}^{i_{0}}\right) \wedge \cdots \wedge A_{\alpha}\left(S^{i_{r}}\right)\right)\right)
$$

and define

$$
\operatorname{THH}^{P}(\tilde{A} ; X)[r]=\operatorname{Ex}^{\infty}\left(\underset{\left(I^{P}\right)^{r+1}}{\operatorname{holim}} G_{r}^{P}(\tilde{A} ; X)\right) .
$$

As $r$ varies this defines a bisimplicial set and the $n$th space of $\operatorname{TH}^{\prime}(\tilde{A})$ is then diagonal simplicial set

$$
\mathrm{TH}^{\prime}(\tilde{A})_{n}=\delta \mathrm{THH}^{P}\left(\tilde{A} ; S^{P}\right),
$$

where $P=\underline{n}$. The discussion above shows that there is a natural isomorphism

$$
\pi_{*} \operatorname{TH}^{\prime}(\tilde{A}) \cong \pi_{*} \operatorname{TH}(\tilde{A}),
$$

where on the left the homotopy groups are calculated on the simplicial level. Moreover, it is also clear that if $A$ is the filtered colimit of rings $A_{\alpha}$, then the canonical map

$$
\underset{\alpha}{\lim _{\alpha}} \operatorname{TH}^{\prime}\left(\tilde{A}_{\alpha}\right) \rightarrow \mathrm{TH}^{\prime}(\tilde{A})
$$

is an isomorphism of quasi-fibrant symmetric spectra of pointed simplicial sets. But taking homotopy groups on the simplicial level commutes with filtered colimits, and hence the claim follows.

\section{HYPERCOHOMOLOGY SPECTRA}

3.1. In his fundamental paper [40], Thomason extends the Godement construction to presheaves of spectra on a site $X$. We briefly recall the construction.

Let $F$ be a presheaf of symmetric spectra of simplicial sets on $X$, that is, a contravariant functor from $X$ to the category of symmetric spectra of simplicial sets. By the stalk of $F$ at the point $x$, we mean the colimit

$$
F_{x}=\underset{\operatorname{nbd}(x)^{\mathrm{op}}}{\lim _{\longrightarrow}} F(U),
$$

where the colimit runs over the opposite category of the category of neighborhoods of $x$ in $X$. The functor $p^{*}$, which to a presheaf associates the collection of its stalks, has a right adjoint $p_{*}$ given by

$$
p_{*}\left(\left\{F_{x}\right\}\right)(U)=\prod_{\operatorname{nbd}(x) \ni U} F_{x} .
$$

Every pair of adjoint functors $\Phi \dashv \Psi$ defines a monad $\Psi \circ \Phi$ and every monad has a cobar construction, [30]. In the case at hand, we denote the monad $p_{*} \circ p^{*}$ by $G$ and call its cobar construction $G \cdot$ the Thomason-Godement construction. The hypercohomology spectrum of the site $X$ with coefficients in the presheaf of symmetric spectra $F$ is then defined as the symmetric spectrum

$$
\mathbb{H}^{\bullet}(X ; F)=\underset{\Delta}{\operatorname{holim}} \Gamma\left(X, G^{\cdot} F\right),
$$


where the homotopy limit is taken over the simplicial category. The unit map of the adjunction $p^{*} \dashv p_{*}$ gives rise to a map

$$
F(X) \stackrel{\eta}{\rightarrow} \mathbb{H}^{\cdot}(X ; F) .
$$

If $F$ is a presheaf of symmetric spectra of spaces on $X$, we define

$$
\mathbb{H}^{\cdot}(X, F)=\underset{\Delta}{\operatorname{holim}} \Gamma\left(X,\left|G^{\cdot} \operatorname{Sin} F\right|\right)
$$

where $|G \cdot \operatorname{Sin} F|$ is the cosimplicial presheaf of symmetric spectra of spaces which in cosimplicial degree $n$ has the presheaf whose value on $U$ is $\left|G^{n} \operatorname{Sin} F(U)\right|$. To see that these definitions are homotopically meaningful, we need the

Lemma 3.1.1. A filtered colimit of quasi-fibrant symmetric spectra of simplicial sets is again quasi-fibrant. Moreover, the homotopy groups of the colimit are equal to the colimit of the homotopy groups.

Proof. Every map from a finite pointed simplicial set (finitely many non-degenerate simplices), to a filtered colimit of pointed simplicial sets factors through a finite stage in the colimit. From this it follows easily that a filtered colimit of fibrant pointed simplicial sets is again fibrant. Since the homotopy groups of a fibrant simplicial set can be calculated using the simplicial spheres, which are finite, the homotopy groups of a filtered colimit of fibrant pointed simplicial sets is the colimit of the homotopy groups. This proves the lemma.

The lemma shows that if $F$ is a presheaf of quasi-fibrant symmetric spectra of simplicial sets, then the stalk $F_{x}$ is again quasi-fibrant and

$$
\pi_{*}\left(F_{x}\right) \cong\left(\pi_{*} F\right)_{x},
$$

the stalk of the presheaf of abelian groups $\pi_{*} F$. Indeed, the category $\operatorname{nbd}(x)^{\text {op }}$ is filtered. More elementary, products preserve quasi-fibrant symmetric spectra and commute with taking homotopy groups, so $G^{\cdot} F$ is a cosimplicial presheaf of quasi-fibrant symmetric spectra and the homotopy groups

$$
\pi_{*}\left(G^{\cdot} F\right) \cong G^{\cdot}\left(\pi_{*} F\right)
$$

is the Godement construction of the presheaf of abelian groups $\pi_{*} F$. If $X$ has enough points then this is a flabby resolution of the sheaf $\tilde{\pi}_{*} F$ associated with the presheaf $\pi_{*} F$ and may thus be used to calculate the sheaf cohomology groups $H^{*}\left(X, \tilde{\pi}_{*} F\right)$. It follows that for a presheaf of quasi-fibrant symmetric spectra (of spaces or simplicial sets) on a site $X$ with enough points, one has a spectral sequence

$$
E_{2}^{s, t}=H^{s}\left(X, \tilde{\pi}_{-t} F\right) \Rightarrow \pi_{-s-t} \mathbb{H}^{\cdot}(X, F),
$$

which converges conditionally in the sense of [2]. Indeed, this is the spectral sequence of $[7, \mathrm{XI}, \S 7]$. Moreover, the edge homomorphism of the spectral sequence coincides with the map induced from the map $\eta$ above.

Proposition 3.1.2. Let $F$. be a pro-presheaf of quasi-fibrant symmetric spectra on $X$ which has enough points. Then there is a conditionally convergent spectral sequence

$$
E_{2}^{s, t}=H_{\text {cont }}^{s}\left(X, \tilde{\pi}_{-t} F .\right) \Rightarrow \pi_{-s-t} \underset{n}{\operatorname{holim}} \mathbb{H}^{\cdot}\left(X, F_{n}\right),
$$

where the groups on the left are the continuous cohomology groups of the pro-sheaf $\tilde{\pi}_{*} F$. in the sense of Jannsen, [25]. 
Proof. The spectral sequence of [7], chap. X, takes the form

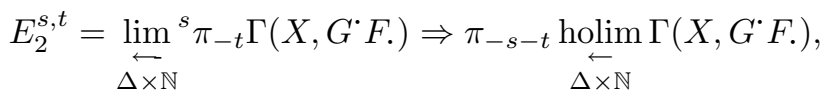

and the identification of the $E^{2}$-term follows from the

Lemma 3.1.3. Let $F$. be a pro-presheaf on $X$ and $\tilde{F}$. its associated pro-sheaf. Then

$$
H_{\text {cont }}^{s}(X, \tilde{F} .)=\underset{\Delta \times \mathbb{N}}{\lim _{\Delta} s} \Gamma(X, G \cdot F .)
$$

provided that $X$ has enough points.

Proof. It follows from definitions that

$$
\lim _{\Delta \times \mathbb{N}} \Gamma\left(X, G^{\cdot} F .\right)=\underset{\overleftarrow{N}}{\lim } \Gamma(X, \tilde{F} .)
$$

for every pro-presheaf on $X$. Hence it suffices to show that for every injective object $I$. in the category of pro-sheaves of abelian groups on $X$,

$$
\lim _{\Delta \times \mathbb{N}} s s\left(X, G \cdot I_{\cdot}\right)=0
$$

when $s>0$. Since limits preserve injectives, we have a Grothendieck spectral sequence

$$
\left.E_{2}^{p, q}=\underset{\overleftarrow{N}}{\lim _{\Delta}} \underset{\overleftarrow{\Delta}}{\left(\lim ^{q}\right.} \Gamma(X, G \cdot I .)\right) \Rightarrow \lim _{\Delta \times \mathbb{N}}{ }^{p+q} \Gamma(X, G \cdot I .)
$$

Now recall from [25] that a pro-sheaf $I$. is injective if and only if each $I_{p}$ is an injective sheaf and each structure map $I_{p} \rightarrow I_{p-1}$ has a section. Hence

$$
\underset{\Delta}{\lim _{\Delta}^{q}} \Gamma\left(X, G \cdot I_{p}\right)=H^{q}\left(X, I_{p}\right)= \begin{cases}\Gamma\left(X, I_{p}\right) & \text { if } q=0 \\ 0 & \text { if } q>0,\end{cases}
$$

and the maps $\Gamma\left(X, I_{p}\right) \rightarrow \Gamma\left(X, I_{p-1}\right)$ have sections such that, e.g. by the MittagLeffler criterion,

$$
\lim _{\mathbb{N}}{ }^{p} \Gamma(X, I .)=0,
$$

for $p>0$. This proves the lemma.

Let $X$ be a scheme and let $K(X)$ be a functorial quasi-fibrant symmetric spectrum model for the non-connective $K$-theory of the category of perfect complexes on $X$. We let $K / p^{n}(X)$ be a functorial fibrant replacement of the smash product of $K(X)$ and a Moore spectrum for $\mathbb{Z} / p^{n}$, where the smash product is formed in the symmetric monoidal category of symmetric spectra, [22]. The homotopy groups is the non-connective $K$-theory with coefficients $K_{*}\left(X ; \mathbb{Z} / p^{n}\right)$. Since the Moore spectrum is finite, [41, theorem 10.3] implies that the canonical map

$$
K / p^{n}(X) \stackrel{\sim}{\longrightarrow} \mathbb{H}^{\cdot}\left(X_{\mathrm{Zar}}, K / p^{n}(-)\right)
$$

is a weak equivalence, at least if the underlying space of $X$ is noetherian of finite dimension. This remains true if we take the homotopy limit over $n$, so we get a weak equivalence

$$
K(X)_{p}^{\wedge} \underset{n}{\stackrel{\sim}{\operatorname{holim}}} \mathbb{H}^{\cdot}\left(X_{\text {Zar }}, K / p^{n}(-)\right) .
$$


The homotopy groups of the $p$-completion $K(X)_{p}^{\wedge}$ are given by Milnor's sequence

$$
0 \rightarrow \underbrace{\lim ^{1}}_{n} K_{i+1}\left(X ; \mathbb{Z} / p^{n}\right) \rightarrow K_{i}\left(X ; \mathbb{Z}_{p}\right) \rightarrow \underbrace{\lim }_{n} K_{i}\left(X ; \mathbb{Z} / p^{n}\right) \rightarrow 0 .
$$

Therefore, the proposition gives a spectral sequence

$$
E_{2}^{s, t}=H_{\text {cont }}^{s}\left(X_{\text {Zar }},\left(\mathcal{K} / p^{\cdot}\right)_{-t}\right) \Rightarrow K_{-s-t}\left(X ; \mathbb{Z}_{p}\right)
$$

which we will call the Zariski descent sequence for $p$-adic $K$-theory.

3.2. Let $\mathrm{Sch} / S$ and Aff $/ S$ denote the categories of all schemes and all affine schemes, respectively, over a base $S$. The functor

$$
U=\operatorname{Spec} A \mapsto \operatorname{TH}(\tilde{A})
$$

defines a presheaf of cyclic quasi-fibrant symmetric spectra on Aff/S. We first study the associated presheaf of abelian groups $\pi_{*} \mathrm{TH}$.

Proposition 3.2.1. Let $A \rightarrow B$ be an étale map of rings. Then the map

$$
B \otimes_{A} \pi_{*} \mathrm{TH}(\tilde{A}) \stackrel{\sim}{\longrightarrow} \pi_{*} \mathrm{TH}(\tilde{B})
$$

induced from the product structure is an isomorphism.

Proof. We deduce the lemma from the corresponding statement for Hochschild homology which is well-known, [13]. Since $A \rightarrow B$ is flat the statement of the lemma is equivalent to the statement that the map of symmetric spectra

$$
\tilde{B} \wedge_{\tilde{A}} \mathrm{TH}(\tilde{A}) \rightarrow \mathrm{TH}(\tilde{B})
$$

is a weak equivalence. By the arithmetic square, it is enough to prove that this map induces isomorphism on homotopy groups with $\mathbb{Z} / p$-coefficients, for all primes $p$, and on rational homotopy groups. Moreover, since the spectra are connective, it will be sufficient to prove the corresponding statements on homology. So let $k$ be a prime field. The skeleton filtration of the cyclic symmetric spectrum $\operatorname{TH}(\tilde{A})$ gives rise to a first quadrant spectral sequence

$$
E^{2}(A)=\mathrm{HH}_{*}\left(\mathcal{A}_{A}\right) \Rightarrow H_{*}(\mathrm{TH}(\tilde{A}) ; k),
$$

where $\mathcal{A}_{A}$ denotes the spectrum homology $H_{*}(\tilde{A} ; k)$. The map $B \otimes_{A} \mathcal{A}_{A} \rightarrow \mathcal{A}_{B}$ induced from the multiplicative structure is an isomorphism since $A \rightarrow B$ is flat. In particular, $\mathcal{A}_{A} \rightarrow \mathcal{A}_{B}$ is étale such that

$$
\mathcal{A}_{B} \otimes_{\mathcal{A}_{A}} \mathrm{HH}_{*}\left(\mathcal{A}_{A}\right) \rightarrow \mathrm{HH}_{*}\left(\mathcal{A}_{B}\right)
$$

is an isomorphism, and moreover,

$$
\mathcal{A}_{B} \otimes_{\mathcal{A}_{A}} \mathrm{HH}_{*}\left(\mathcal{A}_{A}\right)=\left(B \otimes_{A} \mathcal{A}_{A}\right) \otimes_{\mathcal{A}_{A}} \mathrm{HH}_{*}\left(\mathcal{A}_{A}\right)=B \otimes_{A} \mathrm{HH}_{*}\left(\mathcal{A}_{A}\right) .
$$

This shows that $B \otimes_{A} E^{2}(A) \rightarrow E^{2}(B)$ is an isomorphism, and since $A \rightarrow B$ is flat, this persists to $E^{\infty}$. Finally, the filtration of $H_{*}(\mathrm{TH}(\tilde{B}) ; k)$ induced from the spectral sequence is finite in each degree such that

$$
B \otimes_{A} H_{*}(\mathrm{TH}(\tilde{A}) ; k) \rightarrow H_{*}(\mathrm{TH}(\tilde{B}) ; k)
$$

is an isomorphism. This concludes the proof.

The lemma shows that $\pi_{*} \mathrm{TH}(-)$ is a quasi-coherent sheaf on $(\mathrm{Aff} / S)_{\tau}$ in any topology $\tau$ which is coarser than the étale topology. Since quasi-coherent sheaves do 
not have higher sheaf cohomology on affine schemes, the descent spectral sequence collapses showing that

Corollary 3.2.2. For any affine scheme $\operatorname{Spec} A$, the canonical map

$$
\mathrm{TH}(\tilde{A}) \stackrel{\sim}{\longrightarrow} \mathbb{H}^{\cdot}\left((\operatorname{Spec} A)_{\tau} ; \mathrm{TH}\right)
$$

is a weak equivalence of quasi-fibrant symmetric spectra.

The corollary suggests that we take the right hand side of 3.2.2 as our definition of the topological Hochschild homology of a general scheme $X$. Note that a presheaf $F$ on Aff/ $S$ can always be extended to a presheaf on $\mathrm{Sch} / S$ by assigning a scheme $X$ the value $F\left(\Gamma\left(X, \mathcal{O}_{X}\right)\right)$.

Definition 3.2.3. $\mathrm{TH}\left(X_{\tau}\right)=\mathbb{H}^{\cdot}\left(X_{\tau} ; \mathrm{TH}\right)$

The choice of topology is irrelevant. For the morphism of sites $X_{\text {ét }} \rightarrow X_{\tau}$ will induce a weak equivalence

$$
\mathrm{TH}\left(X_{\tau}\right) \stackrel{\sim}{\longrightarrow} \mathrm{TH}\left(X_{\text {ét }}\right),
$$

at least if $X$ is quasi-compact and quasi-separated. Indeed, for affine schemes this is 3.2.2 and the general case follows from this using the fact that hypercohomology has Čech cohomological descent, [40, theorem 1.46].

3.3. We next consider topological cyclic homology. Note that the symmetric spectrum $\mathbb{H}^{\cdot}\left(X_{\tau}, \mathrm{TH}\right)$ has an action by the topological group $\left|\operatorname{Sin} S^{1}\right|$ and hence by the circle $S^{1}$. Moreover, the canonical map

$$
\mathbb{H}^{\cdot}\left(X_{\tau} ; \mathrm{TH}^{C_{r}}\right) \rightarrow \mathbb{H}^{\cdot}\left(X_{\tau} ; \mathrm{TH}\right)^{C_{r}}
$$

is an isomorphism since finite limits commute with realization, filtered colimits and with any functor which has a left adjoint. Hence the restriction and Frobenius maps of (5) and (7) induce similar maps

$$
R_{r}, F_{r}: \mathrm{TH}\left(X_{\tau}\right)^{C_{r s}} \rightarrow \mathrm{TH}\left(X_{\tau}\right)^{C_{s}},
$$

where again $R_{r}$ and $F_{r}$ are maps of quasi-fibrant symmetric spectra, respectively. Following (8) we define pro-symmetric spectra $\mathrm{TR}^{\cdot}\left(X_{\tau} ; p\right)$ and $\mathrm{TC}^{\cdot}\left(X_{\tau} ; p\right)$, and then

$$
\begin{aligned}
& \operatorname{TR}\left(X_{\tau} ; p\right)=\underset{R}{\operatorname{holim}} \operatorname{TR}^{n}\left(X_{\tau} ; p\right) \\
& \operatorname{TC}\left(X_{\tau} ; p\right)=\underset{\longleftarrow}{\operatorname{holim}} \operatorname{TC}^{n}\left(X_{\tau} ; p\right) .
\end{aligned}
$$

This is our definition of the topological cyclic homology of the site $X_{\tau}$. We proceed to show that the choice of topology is irrelevant. The key result we need is the analog of the fundamental cofibration sequence 2.3.1.

Lemma 3.3.1. Let $X$ be a quasi-compact and quasi-separated scheme and let $\tau$ be any topology coarser than the étale topology. Then the canonical map

$$
\mathbb{H}^{\cdot}\left(X_{\tau} ; \mathrm{TH}\right)_{h C_{r}} \stackrel{\sim}{\longrightarrow} \mathbb{H}^{\cdot}\left(X_{\tau} ; \mathrm{TH}_{h C_{r}}\right)
$$

is a weak equivalence. 
Proof. The homotopy orbit spectrum $\mathrm{TH}(E)_{h C_{r}}$ is a fibrant replacement of the symmetric spectrum $\mathrm{TH}(E) \wedge_{C_{r}} E C_{r+}$, where $E C_{r}$ is a contractible space on which $C_{r}$ acts freely. We may choose $E C_{r}$ such that the $k$-skeleton $E_{k} C_{r}$ is finite. This implies that

$$
\mathbb{H}^{\cdot}\left(X_{\tau} ; \mathrm{TH}\right) \wedge_{C_{r}} E_{k} C_{r+} \stackrel{\sim}{\longrightarrow} \mathbb{H}^{\cdot}\left(X_{\tau} ; \mathrm{TH} \wedge_{C_{r}} E_{k} C_{r+}\right)
$$

is a weak equivalence. Moreover, the canonical map

$$
\underset{k}{\operatorname{holim}} \mathbb{H}^{\cdot}\left(X_{\tau} ; \mathrm{TH}\right) \wedge_{C_{r}} E_{k} C_{r+} \stackrel{\sim}{\longrightarrow} \mathbb{H}^{\cdot}\left(X_{\tau} ; \mathrm{TH}\right)_{h C_{r}}
$$

is a weak equivalence, so we have left to show that the same holds for the map

$$
\underset{k}{\operatorname{holim}} \mathbb{H}^{*}\left(X_{\tau} ; \mathrm{TH} \wedge_{C_{r}} E_{k} C_{r+}\right) \rightarrow \mathbb{H}^{*}\left(X_{\tau} ; \mathrm{TH}_{h C_{r}}\right) .
$$

The cofibration sequence of $C_{r}$-spaces

$$
E_{k-1} C_{r+} \longmapsto E_{k} C_{r+} \rightarrow \bigvee_{\alpha} \Sigma^{k} C_{r+}
$$

leads to a cofibration sequence of symmetric spectra

$$
\mathbb{H}^{\cdot}\left(X_{\tau} ; \mathrm{TH} \wedge_{C_{r}} E_{k-1} C_{r+}\right) \rightarrow \mathbb{H}^{\cdot}\left(X_{\tau} ; \mathrm{TH} \wedge_{C_{r}} E_{k} C_{r+}\right) \rightarrow \bigvee_{\alpha} \Sigma^{k} \mathbb{H}^{\cdot}\left(X_{\tau} ; \mathrm{TH}\right),
$$

where the wedge sum on the right is indexed by the set of (equivariant) $k$-cells in $E C_{r}$. Since $X$ is quasi-compact and quasi-separated, the spectrum $\mathbb{H}^{\cdot}\left(X_{\tau} ; \mathrm{TH}\right)$ is bounded below, and hence the connectivity of the wedge sum on the right tends to infinity with $k$, proving the claim.

Proposition 3.3.2. Let $X$ be a quasi-compact and quasi-separated scheme. Then there is a cofibration sequence of quasi-fibrant symmetric spectra

$$
\mathrm{TH}\left(X_{\tau}\right)_{h C_{p^{n}}} \stackrel{N}{\longrightarrow} \mathrm{TR}^{n+1}\left(X_{\tau} ; p\right) \stackrel{R}{\longrightarrow} \mathrm{TR}^{n}\left(X_{\tau} ; p\right),
$$

where $\tau$ may be any topology which is coarser than the étale topology.

Proof. The hypercohomology construction preserves cofibration sequences, so theorem 2.3.1 gives a cofibration sequence

$$
\mathbb{H}^{\cdot}\left(X_{\tau} ; \mathrm{TH}_{h C_{p^{n}}}\right) \stackrel{N}{\longrightarrow} \mathbb{H}^{\cdot}\left(X_{\tau} ; \mathrm{TH}^{C_{p^{n}}}\right) \stackrel{R}{\longrightarrow} \mathbb{H}^{\cdot}\left(X_{\tau} ; \mathrm{TH}^{C_{p^{n-1}}}\right) .
$$

The middle and the right hand term are canonically isomorphic to the terms in the cofibration sequence in the statement, and the lemma identifies the left hand term.

Corollary 3.3.3. For any ring $A$, the canonical map

$$
\mathrm{TC}(\tilde{A} ; p) \stackrel{\sim}{\longrightarrow} \mathrm{TC}\left((\operatorname{Spec} A)_{\tau} ; p\right)
$$

is a weak equivalence of quasi-fibrant symmetric spectra.

Proof. Taking homotopy orbits preserves weak equivalences, so lemma 3.2.2 implies that

$$
\left.\operatorname{TH}(\tilde{A})_{h C_{p^{n}}} \stackrel{\sim}{\longrightarrow} \mathrm{TH}\left((\operatorname{Spec} A)_{\tau}\right)\right)_{h C_{p^{n}}}
$$

is a weak equivalence. It then follows inductively from 3.3.2 that same holds for the presheafs $\mathrm{TR}^{n}(-; p)$, and this in turn implies the statement for $\mathrm{TC}^{n}(-; p)$. The corollary follows by taking homotopy limits. 
Corollary 3.3.4. The morphism of sites $X_{\text {ét }} \rightarrow X_{\tau}$ induces a weak equivalence of quasi-fibrant symmetric spectra

$$
\mathrm{TC}\left(X_{\tau} ; p\right) \stackrel{\sim}{\longrightarrow} \mathrm{TC}\left(X_{\text {ét }} ; p\right),
$$

at least if $X$ is quasi-compact and quasi-separated.

Analogous statements hold for the functor $\mathrm{TR}(-; p)$. Moreover, the diagram of quasi-fibrant symmetric spectra

$$
\mathrm{TC}\left(X_{\tau} ; p\right) \longrightarrow \mathrm{TR}\left(X_{\tau} ; p\right) \underset{F}{\stackrel{R}{\rightleftarrows}} \mathrm{TR}\left(X_{\tau} ; p\right)
$$

is a homotopy equalizer. Let $\mathcal{T R}_{i}$ and $\mathcal{T} \mathcal{C}_{i}$ denote the pro-sheaves on $(\mathrm{Sch} / S)_{\tau}$ associated with the pro-presheaves $\pi_{i} \mathrm{TC}(-; p)$ and $\pi_{i} \mathrm{TR}(-; p)$, respectively. Then the spectral sequence 3.1.2 takes the form

$$
E_{2}^{s, t}=H_{\text {cont }}^{s}\left(X_{\tau} ; \mathcal{T C}_{i}^{\cdot}\right) \Rightarrow \pi_{-s-t} \operatorname{TC}\left(X_{\tau} ; p\right),
$$

where, by the corollary, the spectrum $\operatorname{TC}\left(X_{\tau} ; p\right)$ is independent up to weak equivalence of the choice of topology $\tau$. The spectral sequence, however, depends on the choice of topology. The continuous cohomology of a pro-sheaf $F$. fits in an exact sequence

$$
0 \rightarrow \varliminf_{n}^{\lim ^{1}} H^{s-1}\left(X_{\tau} ; F_{n}\right) \rightarrow H_{\text {cont }}^{s}\left(X_{\tau} ; F .\right) \rightarrow \underset{n}{\lim } H^{s}\left(X_{\tau} ; F_{n}\right) \rightarrow 0 .
$$

The sheaf cohomology of any Zariski sheaf of abelian groups on a scheme $X$ vanishes in dimensions $>\operatorname{dim} X$. It follows that the continuous cohomology of a Zariski pro-sheaf of abelian groups vanishes in dimensions $>\operatorname{dim} X+1$. In particular, the groups $\mathrm{TC}_{i}(X ; p)$ vanish for $i<-\operatorname{dim} X-2$. In fact, they vanish for $i<$ $-\operatorname{dim} X-1$. For the structure maps in the pro-sheaf $\mathcal{T C}_{-1}^{\cdot}$ are surjections and top dimensional sheaf cohomology is right exact, so the relevant derived limit vanishes.

Remark. In [8], Dundas and McCarthy give a definition of topological cyclic homology of an exact category, which in the case of the category of finitely generated projective modules over a ring $A$ recovers the topological cyclic homology of $A$. It would therefore be natural to define topological cyclic homology of a scheme $X$ as the topological cyclic homology of the exact category of algebraic vector bundles on $X$. Such a definition has some advantages. For example, it implies a formula for the topological cyclic homology of a Brauer-Severi variety analogous to the well-known formula in $K$-theory. To show that the Dundas-McCarthy definition agrees with the definition given here, it would be sufficient to show that every covering of $X$ by two Zariski open sets gives rise to a Mayer-Vietoris square. We shall not go into this here, however.

\section{The PRo-Sheaves $\mathrm{TC}_{i}$}

4.1. Throughout this paragraph, $k$ will be a perfect field of characteristic $p>0$, $W$ its ring of $p$-typical Witt vectors and $K$ the field of fractions of $W$.

The de Rham-Witt complex $W \cdot \Omega^{*}$ of Deligne-Illusie, [23], is a complex of propresheaves on $\mathrm{Sch} / k$ which may be characterized as the universal example of a certain algebraic structure, see e. g. $[18, \S 1]$. It is proved in loc. cit. that the 
pro-presheaves $\mathrm{TR}_{*}^{*}(-; p)$ gives another example of this structure such that there is a canonical map of complexes of pro-presheaves

$$
\lambda: W . \Omega^{*} \rightarrow \mathrm{TR}_{*}^{*}(-; p),
$$

where the differential on the right is given by Connes' $B$-operator. Both of these pro-presheaves are in fact pro-sheaves for the étale topology, and hence also for any coarser topology. We also denote the right hand side by $\mathcal{T R}_{*}$. It follows from a theorem of the second author that the canonical map

$$
\lambda: W . \Omega^{*} \stackrel{\sim}{\longrightarrow} \mathcal{T} \mathcal{R}_{*}
$$

is an isomorphism of complexes of pro-presheaves on $\mathrm{Sm} / k$. Indeed, by $[18$, theorem $\mathrm{B}]$ there is a canonical isomorphism

$$
W_{n} \Omega^{i} \otimes \mathbb{Z}\left[\sigma_{n}\right] \stackrel{\sim}{\longrightarrow} T R_{i}^{n},
$$

such that $R\left(\sigma_{n}\right)=p \sigma_{n-1}$ up to a $p$-adic unit and such that the cokernel $C_{n}$ of the canonical map $W_{n} \Omega^{i} \rightarrow \mathcal{T} \mathcal{R}_{i}^{n}$ is isomorphic to $W_{n} \Omega^{i} \otimes \sigma_{n} \mathbb{Z}\left[\sigma_{n}\right]$. Since $W_{n} \Omega^{i}$ is a $\mathbb{Z} / p^{n}$-module and $R\left(\sigma_{n}\right)=p \sigma_{n-1}$, it follows that $C_{2 n} \rightarrow C_{n}$ is the zero map, and hence the cokernel pro-sheaf is Mittag-Leffler zero. The kernel is trivial.

Consider the map $d \log : \mathbb{G}_{m} \rightarrow W_{n} \Omega^{1}$ of presheaves on $\mathrm{Sm} / k$ which takes a section $x$ to $d \underline{x}_{n} / \underline{x}_{n}$, where ${ }_{n}: \mathbb{G}_{m} \rightarrow W_{n}\left(\mathbb{G}_{a}\right)$ is the Teichmüller section. Using the product on $W_{n} \Omega^{*}$, we get a map of presheaves

$$
\mathbb{G}_{m}^{\otimes i} \rightarrow W_{n} \Omega^{i} .
$$

Both of these presheaves are in fact sheaves on $(\mathrm{Sm} / k)$ ét and the image sheaf is denoted by $\nu_{n}(i)$, and also $W_{n} \Omega_{\log }^{i}$, and called the $i$ th logarithmic de Rham-Witt sheaf. In other words, $\nu_{n}(i)$ is the subsheaf of the sheaf $W_{n} \Omega^{i}$ on $(\mathrm{Sm} / k)_{\text {ét }}$ which is generated locally for the étale topology by sections of the form $d \log \underline{x}_{1} \wedge \ldots \wedge d \log \underline{x}_{i}$. By Gros-Suwa [17], the sheaves $\nu_{n}(i)$ have a Gersten resolution

$$
0 \rightarrow \nu_{n}(i) \rightarrow \bigoplus_{x \in X^{(0)}} i_{*} \nu_{n}(i)(k(x)) \rightarrow \bigoplus_{x \in X^{(1)}} i_{*} \nu_{n}(i-1)(k(x)) \rightarrow \ldots,
$$

which in particular shows that $H_{\text {cont }}^{i}\left(X_{\text {Zar }}, \nu \cdot(j)\right)=0$ for $i>j$. As $n$ varies we get a pro-sheaf $\nu .(i)$ on $(\mathrm{Sm} / k)_{\text {ét }}$, and it is proved in [23, I 5.7.2] that the sequences

$$
0 \rightarrow \nu .(i) \rightarrow W . \Omega^{i} \stackrel{1-F}{\longrightarrow} W . \Omega^{i} \rightarrow 0
$$

are exact. Here $F$ is the Frobenius operator on the de Rham-Witt complex. It is related to the geometric Frobenius by $\phi=p^{i} F$.

Theorem 4.1.1. The pro-sheaves $\mathcal{T C}_{i}$ and $\nu .(i)$ on $(\mathrm{Sm} / k)_{\text {ét }}$ are isomorphic such that the descent spectral sequence takes the form

$$
E_{2}^{s t}=H_{\text {cont }}^{s}\left(X_{\text {ét }}, \nu \cdot(-t)\right) \Rightarrow \mathrm{TC}_{-s-t}(X ; p) .
$$

Moreover, the spectral sequence degenerates rationally with naturally split filtration.

Proof. The isomorphism (14) identifies the long-exact homotopy sequence

$$
\cdots \rightarrow \mathcal{T C}_{i} \rightarrow \mathcal{T R}_{i} \stackrel{1-F}{\longrightarrow} \mathcal{T R}_{i} \rightarrow \mathcal{T C}_{i-1} \rightarrow \ldots
$$

with the short-exact sequences (15). This proves the first claim and the spectral sequence is then given by (12). To see that spectral sequence degenerates rationally, we consider the action by the geometric Frobenius endomorphism $\phi_{X}$ on the spectral 
sequence. But $\phi_{X}$ acts like $p^{i}$ on $\nu .(i)$, and by naturality of the spectral sequence, the differentials commute with $\phi_{X}$. Hence

$$
d_{r}^{s,-t}\left(p^{t} x\right)=p^{t+r-1} d_{r}^{s,-t}(x)
$$

such that $p^{t}\left(1-p^{r-1}\right) d_{r}^{s,-t}=0$.

Remark. On a smooth scheme $X$, the de Rham-Witt pro-sheaves $W . \Omega^{i}$ vanish above the dimension of $X$ and the sequence (15) then shows that same holds for the prosheaves $\nu .(i)$. Hence the spectral sequence shows that

$$
\mathrm{TC}_{i}(X ; p)=0,
$$

when $i>\operatorname{dim} X$ and $X$ is smooth.

Lemma 4.1.2. Let $X$ be a smooth affine scheme. Then $H_{\text {cont }}^{s}\left(X_{\tau}, W \cdot \Omega^{i}\right)=0$, for $s>0$, in either the Zariski or étale topology, and $H_{\text {cont }}^{s}\left(X_{\text {ét }}, \nu .(i)\right)=0$, for $s>1$.

Proof. On an affine scheme $X$, the cohomology of a quasi-coherent sheaf of $\mathcal{O}_{X^{-}}$ modules vanishes in positive degrees. This holds in both the Zariski and étale topology. Let $W_{n}(X)$ be the scheme which has $X$ as its underlying topological space and $W_{n}\left(\mathcal{O}_{X}\right)$ as its structure sheaf. Then $W_{n} \Omega^{i}$ is a quasi-coherent sheaf of $W_{n}\left(\mathcal{O}_{X}\right)$-modules. It follows that the higher sheaf cohomology of $W_{n} \Omega_{X}^{i}$ vanishes. Hence the first statement follows from (13), and the second statement is a consequence of the first statement and (15).

Consider the morphism of sites

$$
\epsilon:(\mathrm{Sm} / k)_{\text {ét }} \rightarrow(\mathrm{Sm} / k)_{\mathrm{Zar}}
$$

and denote the restriction $\epsilon_{*} \nu_{n}(i)$ again by $\nu .(i)$. By lemma 4.1.2, we get an exact sequence of Zariski pro-sheaves

$$
0 \rightarrow \nu \cdot(i) \rightarrow W . \Omega^{i} \stackrel{F-1}{\longrightarrow} W . \Omega^{i} \rightarrow R^{1} \epsilon_{*} \nu .(i) \rightarrow 0,
$$

and the higher derived images $R^{s} \epsilon_{*} \nu$. (i) vanish for $s>1$. By (14), the canonical map $W . \Omega^{i} \rightarrow \mathcal{T} \mathcal{R}_{i}^{i}$ is an isomorphism of Zariski pro-sheaves on $\mathrm{Sm} / k$, and comparing the sequences above to the long-exact homotopy sequence

$$
\cdots \rightarrow \mathcal{T C}_{i}^{\cdot} \rightarrow \mathcal{T R}_{i} \stackrel{1-F}{\longrightarrow} \mathcal{T R}_{i} \rightarrow \mathcal{T C}_{i}^{\cdot} \rightarrow \ldots
$$

we get short sequences

$$
0 \rightarrow R^{1} \epsilon_{*} \nu .(i+1) \rightarrow \mathcal{T C}_{i} \stackrel{\rho}{\rightarrow} \nu .(i) \rightarrow 0
$$

of pro-sheaves on $(\mathrm{Sm} / k)_{\mathrm{Zar}}$.

Proposition 4.1.3. There is a natural isomorphism

$$
H_{\text {cont }}^{0}\left(X_{\text {Zar }}, R^{1} \epsilon_{*} \nu .(1)\right) \cong T_{p} \operatorname{Br}(X),
$$

where the right term is the Tate module of the (cohomological) Brauer group of $X$.

Proof. We have a short exact sequence of étale sheaves

$$
0 \rightarrow \mathbb{G}_{m} \stackrel{p^{n}}{\longrightarrow} \mathbb{G}_{m} \stackrel{d \log }{\longrightarrow} \nu_{n}(1) \rightarrow 0
$$


and Hilbert's theorem 90 and lemma 4.1.2 shows that associated long exact sequence of higher derived image sheaves takes the form

$$
0 \rightarrow R^{1} \epsilon_{*} \nu_{n}(1) \rightarrow R^{2} \epsilon_{*} \mathbb{G}_{m} \stackrel{p^{n}}{\longrightarrow} R^{2} \epsilon_{*} \mathbb{G}_{m} \rightarrow 0 .
$$

It follows that $H^{0}\left(X_{\mathrm{Zar}}, R^{1} \epsilon_{*} \nu_{n}(1)\right) \cong p^{n} H^{0}\left(X_{\mathrm{Zar}}, R^{2} \epsilon_{*} \mathbb{G}_{m}\right)$, and finally, Hilbert's theorem 90 and the vanishing of $H^{2}\left(X_{\text {Zar }}, \mathbb{G}_{m}\right)$ shows that edge homomorphism in the change of topology spectral sequence induces an isomorphism

$$
H^{0}\left(X_{\mathrm{Zar}}, R^{2} \epsilon_{*} \mathbb{G}_{m}\right) \cong H^{2}\left(X_{\text {ét }}, \mathbb{G}_{m}\right)=\operatorname{Br}(X) .
$$

The stated isomorphism now follows from the short exact sequence for continuous cohomology, (13).

4.2. The Milnor $K$-groups of a field $E$ are defined as

$$
K_{*}^{M}(E)=T^{*}\left(E^{\times}\right) /\left(a \otimes(1-a) \mid a \in E^{\times}-\{1\}\right),
$$

the tensor algebra on the units modulo the ideal generated by the Steinberg relation. If $E$ is a field of characteristic $p>0$, one has the map

$$
d \log : K_{i}^{M}(E) \rightarrow W_{n} \Omega_{E}^{i}
$$

induced via products from $d \log x=d \underline{x}_{n} / \underline{x}_{n}$. It factors through the Steinberg relation because $W_{n} \Omega_{\mathbb{F}_{p}[x]}^{2}=0$. It is easily checked that $(R-F) \circ d \log =0$, and by Bloch-Gabber-Kato, [1], the induced map

$$
d \log : K_{i}^{M}(E) / p^{n} \rightarrow \nu_{n}(i)(E)
$$

is an isomorphism. It is known that the groups $K_{i}^{M}(E)$ are $p$-torsion free. This was proved by Suslin, [39], when $i=2$, and by Izhboldin, [24], in general. Recently, the first author and M. Levine have shown

Theorem 4.2.1. ([11]) Let E be a field of characteristic $p>0$. Then the canonical map

$$
\iota: K_{i}^{M}(E) / p^{n} \rightarrow K_{i}\left(E, \mathbb{Z} / p^{n}\right)
$$

is an isomorphism.

The cyclotomic trace map induces a map of pro-sheaves on $(\mathrm{Sch} / k)_{\mathrm{Zar}}$ :

$$
\operatorname{trc}:\left(\mathcal{K} / p^{\cdot}\right)_{*} \rightarrow \mathcal{T} \mathcal{C}_{*}^{\cdot},
$$

where we remember, pro-sheaves are considered up to the Serre subcategory of Mittag-Leffler zero pro-sheaves. Indeed, the cyclotomic trace gives, upon smashing with the $\bmod p^{\cdot}$ Moore spectrum, a map of pro-presheaves of symmetric spectra on $\mathrm{Sch} / k$,

$$
K(-) / p^{\cdot} \rightarrow \mathrm{TC}^{\cdot}(-; p) / p^{\bullet},
$$

and the map of homotopy groups induced from the reduction map

$$
\mathrm{TC}^{\cdot}(-; p) \rightarrow \mathrm{TC}^{\cdot}(-; p) / p
$$

is an isomorphism of pro-presheaves of abelian groups on Sch/ $k$. Let us explain the last statement in more detail. We have maps of cofibration sequences of symmetric 
spectra

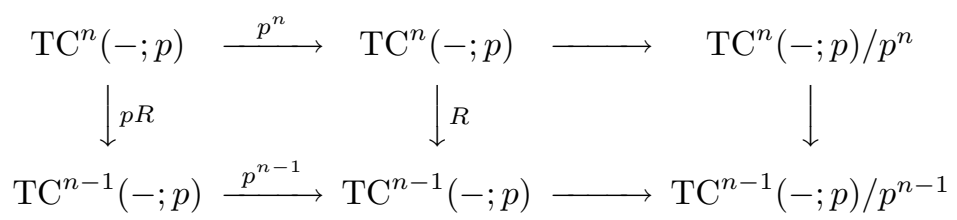

and the statement is then equivalent to the pro-presheaf of homotopy groups associated with the left hand maps being Mittag-Leffler zero. But the groups $\pi_{*} \mathrm{TR}^{n}(-; p)$ are $p^{n}$-torsion and the sequence

$$
\cdots \rightarrow \pi_{*} \mathrm{TC}^{n}(-; p) \rightarrow \pi_{*} \mathrm{TR}^{n}(-; p) \stackrel{R-F}{\longrightarrow} \pi_{*} \mathrm{TR}^{n-1}(-; p) \rightarrow \ldots
$$

shows that the groups $\pi_{*} \mathrm{TC}^{n}(-; p)$ are $p^{2 n-1}$-torsion.

Theorem 4.2.2. The composition of maps of pro-sheaves on $(\mathrm{Sm} / k)_{\mathrm{Zar}}$,

$$
\left(\mathcal{K} / p^{\cdot}\right)_{i} \stackrel{\operatorname{trc}}{\longrightarrow} \mathcal{T} \mathcal{C}_{i} \stackrel{\rho}{\rightarrow} \nu \cdot(i),
$$

is an isomorphism.

Proof. It is enough to show that the composite induces an isomorphism of stalks at the points of $(\mathrm{Sm} / k)_{\mathrm{Zar}}$, and since the functors in question all commute with filtered colimits, this is equivalent to showing that for any essentially smooth local $k$-algebra $A$, the composite map

$$
K_{i}\left(A, \mathbb{Z} / p^{\cdot}\right) \stackrel{\operatorname{trc}}{\longrightarrow} \mathrm{TC}_{i}(A ; p) \stackrel{\rho}{\rightarrow} \nu \cdot(i)(A)
$$

is an isomorphism of pro-abelian groups up to Mittag-Leffler zero objects. To this end, we first use Gersten resolutions together with (17) and 4.2.1 to produce an isomorphism between $K_{i}\left(A, \mathbb{Z} / p^{*}\right)$ and $\nu \cdot(i)(A)$. We then show that the map above induces the isomorphism.

Let $U=\operatorname{Spec} A$ and let $x$ be a point of codimension 1 on $U$. Let $k(U)$ be the function field of $U$ and let $k(x)$ be the residue field at $x$. Let $R_{i}$ denote any of the functors $K_{i}\left(-, \mathbb{Z} / p^{n}\right), K_{i}^{M} / p^{n}$ and $\nu_{n}(i)$. Then one has residue homomorphisms

$$
R_{i}(k(U)) \stackrel{\partial_{x}}{\longrightarrow} R_{i-1}(k(x)),
$$

and these are compatible up to a sign with the maps $\iota$ and $d \log$ such that the following diagram commutes up to a sign:

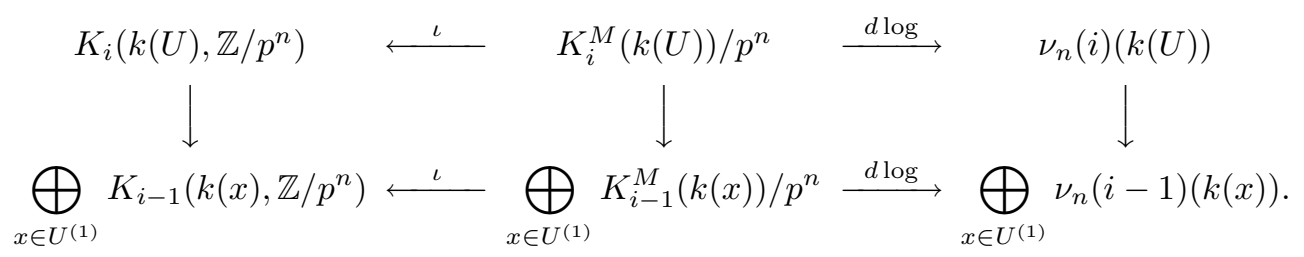

Moreover, the inclusion of the generic point $\operatorname{Spec} k(U) \rightarrow U$ induces isomorphisms of the kernel of the left vertical map and $K_{i}\left(U, \mathbb{Z} / p^{n}\right)$ and of the right vertical map and $\nu_{n}(i)(U)$. The first of these statements is proved analogously to $[37, \S 7$, 5.11], and the second statement is proved in [17]. Now the right horizontal maps are isomorphism by (17) and the left horizontal maps are isomorphisms by 4.2.1. It follows that the abelian groups $K_{i}\left(U, \mathbb{Z} / p^{n}\right)$ and $\nu_{n}(i)(U)$ are isomorphic, and hence so are the corresponding pro-abelian groups. 
It remains to show that the composite map listed in the beginning of the proof induces the isomorphism. By naturality, it will suffice to prove that the following diagram of pro-abelian groups commutes:

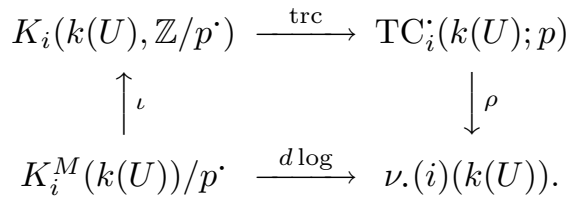

We write $E=k(U)$ and embed this diagram in the larger diagram



Since the lower left hand horizontal map is epi, the lower right hand horizontal map is mono and the right hand vertical map is an isomorphism, we may instead show that the outer diagram commutes. We prove in the appendix that the cyclotomic trace is multiplicative, thus reducing us to showing that the diagram commutes for $i=1$. Since the differential on the de Rham-Witt complex and Connes' $B$ operator are compatible under the map $\lambda$, the commutativity would be trivial was trc replaced by the map $B \log$. Hence the following lemma finishes the proof.

Lemma 4.2.3. Let $A$ be any commutative ring, let $\operatorname{tr}: K_{1}(A) \rightarrow \mathrm{TR}_{1}(A ; p)$ be the trace and let $\iota_{1}: A^{\times} \rightarrow K_{1}(A)$ be the inclusion of the units. Then

$$
\operatorname{tr} \circ \iota_{1}=-B \log ,
$$

where $B \log x=B \underline{x} \cdot \underline{x}^{-1}$.

Before we prove the lemma, we derive three immediate corollaries of the theorem.

Corollary 4.2.4. There is a canonical isomorphism of pro-sheaves on $(\mathrm{Sm} / k)_{\mathrm{Zar}}$,

$$
\mathcal{T} \mathcal{C}_{i} \cong \nu \cdot(i) \oplus R^{1} \epsilon_{*} \nu .(i+1),
$$

and the cyclotomic trace corresponds to the inclusion of the first summand.

Proof. Indeed, theorem 4.2.2 identifies the pro-sheaves $\nu .(i)$ and $\left(\mathcal{K} / p^{\circ}\right)_{i}$ and shows that the cyclotomic trace splits the sequence (16).

Corollary 4.2.5. The map of pro-sheaves on $(\mathrm{Sm} / k)$ ét induced from the cyclotomic trace,

$$
\operatorname{trc}:\left(\mathcal{K} / p^{*}\right)_{i} \stackrel{\sim}{\longrightarrow} \mathcal{T} \mathcal{C}_{i}
$$

is an isomorphism.

Proof. Sheafifying the composite map of Zariski pro-sheaves in the statement of theorem 4.2.2 for the étale topology, the map $\rho$ becomes an isomorphism by corollary 4.1.1. Since the composite is also an isomorphism, the claim follows.

Corollary 4.2.6. If $X$ is a smooth scheme over $k$, then

$$
\mathrm{TC}(X ; p) \simeq \mathbb{H}^{\cdot}\left(X_{\text {ét }}, K(-)\right)_{p}^{\wedge},
$$

at least if $X$ is quasi-compact and quasi-separated. 
Proof. The map of hypercohomology spectra induced by the cyclotomic trace,

$$
\underset{\mathbb{N}}{\operatorname{holim}} \mathbb{H}^{*}\left(X_{\text {ét }}, K / p^{*}(-)\right) \stackrel{\sim}{\longrightarrow} \underset{\mathbb{N}}{\operatorname{holim}} \mathbb{H}^{\cdot}\left(X_{\text {ét }}, \mathrm{TC}^{\cdot}(-; p)\right)
$$

is a weak equivalence. For by the previous corollary, the map of $E_{2}$-terms of the corresponding descent spectral sequences is an isomorphism, and for $X$ quasi-compact and quasi-separated, lemma 4.1.2 and the fact that hypercohomology has Cech cohomological descent shows that the spectral sequences are concentrated in the region $s \leq N$, for some $N$, and hence converges strongly. Now

$$
\mathrm{TC}(E ; p) \stackrel{\sim}{\longrightarrow} \underset{\mathbb{N}}{\operatorname{holim}} \mathbb{H}^{*}\left((\operatorname{Spec} E)_{\text {ét }}, \mathrm{TC}^{\cdot}(-; p)\right)
$$

by étale descent for topological cyclic homology, 3.3.3, and since the mod $p$ Moore spectrum is finite

$$
\mathbb{H}^{*}\left((\operatorname{Spec} E)_{\text {ét }}, K / p^{\cdot}(-)\right) \simeq \mathbb{H}^{\bullet}\left((\operatorname{Spec} E)_{\text {ét }}, K(-)\right) / p^{*},
$$

see e. g. [34, 3.10]. But by definition

$$
\underset{\overleftarrow{N}}{\operatorname{holim}} \mathbb{H}^{\cdot}\left((\operatorname{Spec} E)_{\text {ét }}, K(-)\right) / p^{\cdot}=\mathbb{H}^{\cdot}\left((\operatorname{Spec} E)_{\text {ét }}, K(-)\right)_{p}^{\wedge}
$$

and the claim follows.

Example. The last corollary is also valid for any field $E$ of characteristic $p>0$, and then states that

$$
\left.\mathrm{TC}(E ; p) \simeq \underset{\underset{\alpha}{\operatorname{holim}}}{\alpha} K\left(E_{\alpha}\right)^{h G_{\alpha}}\right)_{p}^{\wedge},
$$

where the homotopy runs over the finite extensions $E_{\alpha} / E$ in a separable closure $E^{s}$ and $G_{\alpha}$ is the Galois group of $E_{\alpha}$. Indeed, [40, Example 1.50] shows that for any presheaf $F$ of quasi-fibrant symmetric spectra on (Spec $E$ )ét,

$$
\mathbb{H}^{*}\left((\operatorname{Spec} E)_{\text {ét }}, F\right) \simeq \underset{\alpha}{\underset{\alpha}{\operatorname{holim}}} F\left(E_{\alpha}\right)^{h G \alpha} .
$$

To see that the corollary holds for fields, it is enough to show that the cyclotomic trace induces an isomorphism

$$
H_{\text {cont }}^{s}\left((\operatorname{Spec} E)_{\text {ét }},\left(\mathcal{K} / p^{*}\right)_{-t}\right) \stackrel{\sim}{\longrightarrow} H_{\text {cont }}^{s}\left((\operatorname{Spec} E)_{\text {ét }}, \mathcal{T} \mathcal{C}_{-t}^{\cdot}\right),
$$

and to this end, we write $E$ as the filtered colimit of the smooth $\mathbb{F}_{p^{-}}$algebras $A_{\alpha}$ and consider the diagram

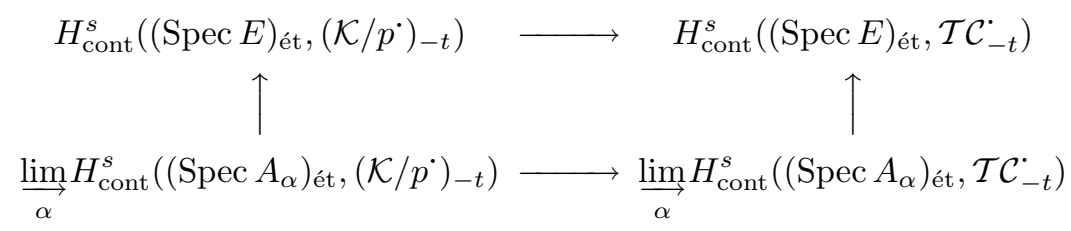

The lower horizontal map is an isomorphism by corollary 4.2 .5 and the vertical maps are isomorphisms by continuity of étale cohomology, [31, III lemma 1.16], and (13).

Proof of the lemma. We begin the proof by recalling the definitions of the maps in the statement. For typographical reasons, we shall shorten notation and write $T$ 
instead of THH. The restriction of the cyclotomic trace is the map of components induced from the composition

$$
G L_{1}(A) \rightarrow \Omega B G L(A)^{+} \stackrel{\operatorname{trc}}{\longrightarrow} \underset{n}{\operatorname{holim}} \Omega^{n+1} \mathrm{TH}(A)_{n}^{C_{r}} .
$$

This map in turn may be identified with the composite

$$
\begin{aligned}
G L_{1}(A) & \stackrel{\tilde{\iota}_{1}}{\longrightarrow} \Omega\left|N G L_{1}(A)\right| \stackrel{I}{\rightarrow} \Omega\left|N^{\mathrm{cy}} G L_{1}(A)\right| \stackrel{\Delta_{r}}{\longrightarrow} \Omega\left|\left(\operatorname{sd}_{r} N^{\mathrm{cy}} G L_{1}(A)\right)^{C_{r}}\right| \\
& \stackrel{j}{\rightarrow} \Omega\left|\left(\operatorname{sd}_{r} N_{\wedge}^{\mathrm{cy}}(A)\right)^{C_{r}}\right| \stackrel{\tilde{\lambda}_{l, 0}}{\longrightarrow} \Omega^{l+1}\left|\left(\operatorname{sd}_{r} T^{\mathbf{l}}\left(\tilde{A} ; S^{l}\right)[-]\right)^{C_{r}}\right| .
\end{aligned}
$$

Here the first map is the adjoint of the inclusion of the 1-skeleton, the second map is induced from the simplicial map

$$
I: N G L_{1}(A) \rightarrow N^{\mathrm{cy}} G L_{1}(A)
$$

given in simplicial degree $k$ by

$$
I\left(x_{1}, \cdots, x_{k}\right)=\left(x_{k}^{-1} \ldots x_{1}^{-1}, x_{1}, \ldots, x_{k}\right),
$$

the third map is the diagonal isomorphism, the fourth map is induced from the inclusion of the units in the underlying multiplicative (pointed) monoid $A$ and the last map is the adjoint of the symmetric spectrum structure map.

We next recall the definition of the map $B \log$ from $[18, \S 1]$. The Teichmüller section

$$
\text { _ }: A \rightarrow \pi_{0} \operatorname{TH}(\tilde{A})^{C_{r}}
$$

is the map of components induced from the composite

$$
A \stackrel{\iota_{0}}{\longrightarrow}\left|N_{\wedge}^{\mathrm{cy}}(A)\right| \stackrel{\Delta_{r}}{\longrightarrow}\left|\left(\operatorname{sd}_{r} N_{\wedge}^{\mathrm{cy}}(A)\right)^{C_{r}}\right| \stackrel{\tilde{\lambda}_{n, 0}}{\longrightarrow} \Omega^{n}\left|\left(\operatorname{sd}_{r} T^{\mathbf{n}}\left(\tilde{A} ; S^{n}\right)[-]\right)^{C_{r}}\right|,
$$

where the first map is the inclusion of the 0-skeleton and the remaining maps are as above. To define the $B$-operator

$$
B: \pi_{*} \mathrm{TH}(A)^{C_{r}} \rightarrow \pi_{*+1} \mathrm{TH}(A)^{C_{r}},
$$

we let $[\sigma] \in \pi_{1}^{S}\left(S_{+}^{1}\right)$ be the class which reduces to (id, 0 ) under the isomorphism

$$
\pi_{1}^{S}\left(S_{+}^{1}\right) \cong \pi_{1}^{S}\left(S^{1}\right) \oplus \pi_{1}^{S}\left(S^{0}\right) \cong \mathbb{Z}\langle\mathrm{id}\rangle \oplus \mathbb{Z} / 2\langle\eta\rangle
$$

induced from the maps

$$
S^{1} \stackrel{p_{1}}{\longleftarrow} S_{+}^{1} \stackrel{p_{0}}{\longrightarrow} S^{0}
$$

which identify the extra base point with $1 \in S^{1}$ and collapses $S^{1}$ to the non-base point in $S^{0}$, respectively. Let $\sigma: S^{m+1} \rightarrow S^{m} \wedge S_{+}^{1}$ be a representative of $[\sigma]$. Then $B$ is the map of homotopy groups induced from the composite

$$
\begin{aligned}
& \Omega^{n}\left|\left(\operatorname{sd}_{r} T^{\mathbf{n}}\left(\tilde{A} ; S^{n}\right)[-]\right)^{C_{r}}\right| \stackrel{\sigma \wedge-}{\longrightarrow} \Omega^{l+1}\left(S^{m} \wedge S_{+}^{1} \wedge\left|\left(\operatorname{sd}_{r} T^{\mathbf{n}}\left(\tilde{A} ; S^{n}\right)\right)^{C_{r}}\right|\right) \\
& \stackrel{\omega}{\longrightarrow} \Omega^{l+1}\left(S^{m} \wedge\left|\left(\operatorname{sd}_{r} T^{\mathbf{n}}\left(\tilde{A} ; S^{n}\right)\right)^{C_{r}}\right|\right) \stackrel{\lambda_{m, n}}{\longrightarrow} \Omega^{l+1}\left|\left(\operatorname{sd}_{r} T^{\mathbf{l}}\left(\tilde{A} ; S^{l}\right)\right)^{C_{r}}\right|,
\end{aligned}
$$

where we have written $l=m+n$ and where $\omega$ is the action map.

Finally, the product

$$
\pi_{*} \operatorname{TH}(\tilde{A})^{C_{r}} \otimes \pi_{*} \operatorname{TH}(\tilde{A})^{C_{r}} \rightarrow \pi_{*} \operatorname{TH}(\tilde{A})^{C_{r}}
$$

is the external product followed by the map of homotopy groups induced from

$$
\Omega^{l}\left|\left(\operatorname{sd}_{r} T^{\mathbf{m}}\left(\tilde{A} ; S^{m}\right)\right)^{C_{r}} \wedge\left(\operatorname{sd}_{r} T^{\mathbf{n}}\left(\tilde{A} ; S^{n}\right)\right)^{C_{r}}\right| \stackrel{\operatorname{sd}_{r} \mu_{m, n}}{\longrightarrow} \Omega^{l}\left|\left(\operatorname{sd}_{r} T^{\mathbf{l}}\left(\tilde{A} ; S^{l}\right)\right)^{C_{r}}\right|,
$$


where again $l=m+n$ and where $\mu_{m, n}$ are defined the multiplication maps defined in $[20,2.7 .1]$. It will be sufficient to know the formula for $\mu_{0,0}$. This is the simplicial, or in fact cyclic, map

$$
\mu_{0,0}: N_{\wedge}^{\mathrm{cy}}(A) \wedge N_{\wedge}^{\mathrm{cy}}(A) \rightarrow N_{\wedge}^{\mathrm{cy}}(A)
$$

given by in simplicial degree $k$ by

$$
\mu_{0,0}\left(x_{0}, \ldots, x_{k} ; y_{0}, \ldots, y_{k}\right)=\left(x_{0} y_{0}, \ldots, x_{k} y_{k}\right) .
$$

Note that we need that the $\operatorname{ring} A$ be commutative for this formula to define a simplicial map.

Let us now go back to the trace map. The map $G L_{1}(A) \rightarrow \pi_{1} \operatorname{TH}(\tilde{A})^{C_{r}}$ is equal to the map of components induced from the composite of the adjunction

$$
G L_{1}(A) \rightarrow \Omega^{l+1}\left(S^{l+1} \wedge G L_{1}(A)\right)
$$

and the map of $(l+1)$ st loop spaces induced from

$$
\begin{aligned}
& S^{l+1} \wedge G L_{1}(A) \stackrel{\iota_{1}}{\longrightarrow} S^{l} \wedge\left|N G L_{1}(A)\right| \stackrel{I}{\rightarrow} S^{l} \wedge\left|N^{\text {cy }} G L_{1}(A)\right| \\
& \stackrel{j}{\longrightarrow} S^{l} \wedge\left|N_{\wedge}^{\text {cy }}(A)\right| \stackrel{\Delta_{r}}{\longrightarrow} S^{l} \wedge\left|\operatorname{sd}_{r} N_{\wedge}^{\text {cy }}(A)^{C_{r}}\right| \stackrel{\tilde{\lambda}_{l, 0}}{\longrightarrow}\left|\left(\operatorname{sd}_{r} T^{l}\left(\tilde{A} ; S^{l}\right)[-]\right)^{C_{r}}\right| .
\end{aligned}
$$

Indeed, this just amounts to saying that the diagram of simplicial sets

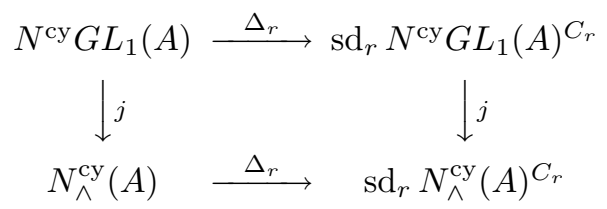

commutes. The map $B \log : G L_{1}(A) \rightarrow \pi_{1} \mathrm{TH}(\tilde{A})^{C_{r}}$ is the map of components induced from the composite of $(19)$ and the map of $(l+1)$ st loop spaces induced from

$$
\begin{aligned}
S^{l+1} \wedge & G L_{1}(A) \stackrel{\nabla}{\longrightarrow} S^{l+1} \wedge A \wedge A \stackrel{\sigma}{\longrightarrow} S_{+}^{1} \wedge S^{l} \wedge A \wedge A \\
& \stackrel{\iota_{0} \wedge \iota_{0}}{\longrightarrow} S_{+}^{1} \wedge S^{l} \wedge\left|N_{\wedge}^{\mathrm{cy}}(A)\right| \wedge\left|N^{\mathrm{cy}} \wedge(A)\right| \\
& \stackrel{\Delta_{r} \wedge \Delta_{r}}{\longrightarrow} S_{+}^{1} \wedge S^{l} \wedge\left|\operatorname{sd}_{r} N_{\wedge}^{\mathrm{cy}}(A)^{C_{r}}\right| \wedge\left|\operatorname{sd}_{r} N^{\mathrm{cy}} \wedge(A)^{C_{r}}\right| \\
& \stackrel{\lambda_{m, 0} \wedge \lambda_{n, 0}}{\longrightarrow} S_{+}^{1} \wedge\left|\left(\operatorname{sd}_{r} T^{\mathbf{m}}\left(\tilde{A} ; S^{m}\right)\right)^{C_{r}}\right| \wedge\left|\left(\operatorname{sd}_{r} T^{\mathbf{n}}\left(\tilde{A} ; S^{n}\right)\right)^{C_{r}}\right| \\
& \stackrel{\omega \wedge \text { id }}{\longrightarrow}\left|\left(\operatorname{sd}_{r} T^{\mathbf{m}}\left(\tilde{A} ; S^{m}\right)\right)^{C_{r}}\right| \wedge\left|\left(\operatorname{sd}_{r} T^{\mathbf{n}}\left(\tilde{A} ; S^{n}\right)\right)^{C_{r}}\right| \\
& \stackrel{\operatorname{sd}_{r} \mu_{m, n}}{\longrightarrow}\left|\left(\operatorname{sd}_{r} T^{\mathbf{l}}\left(\tilde{A} ; S^{l}\right)\right)^{C_{r}}\right|,
\end{aligned}
$$

where $l=m+n$ and where $\nabla(x)=x \wedge x^{-1}$. We claim that this last map is equal to the composition

$$
\begin{aligned}
S^{l+1} \wedge & G L_{1}(A) \stackrel{\nabla}{\longrightarrow} S^{l+1} \wedge A \wedge A \stackrel{\sigma}{\longrightarrow} S_{+}^{1} \wedge S^{l} \wedge A \wedge A \\
& \stackrel{\iota_{0} \wedge \iota_{0}}{\longrightarrow} S_{+}^{1} \wedge S^{l} \wedge\left|N_{\wedge}^{\mathrm{cy}}(A)\right| \wedge\left|N^{\mathrm{cy}} \wedge(A)\right| \\
& \stackrel{\omega \wedge \mathrm{id}}{\longrightarrow} S^{l} \wedge\left|N_{\wedge}^{\mathrm{cy}}(A)\right| \wedge\left|N^{\mathrm{cy}} \wedge(A)\right| \\
& \stackrel{\mu_{0,0}}{\longrightarrow} S^{l} \wedge\left|N_{\wedge}^{\mathrm{cy}}(A)\right| \\
& \stackrel{\Delta_{r}}{\longrightarrow} S^{l} \wedge\left|\operatorname{sd}_{r} N_{\wedge}^{\mathrm{cy}}(A)^{C_{r}}\right| \stackrel{\lambda_{l, 0}}{\longrightarrow}\left|\left(\operatorname{sd}_{r} T^{\mathrm{l}}\left(\tilde{A} ; S^{l}\right)[-]\right)^{C_{r}}\right| .
\end{aligned}
$$


Indeed, the fact that $\mathrm{TH}(\tilde{A})^{C_{r}}$ is a symmetric spectrum with an $S^{1}$-action makes the appropriate diagrams commute. Comparing this to the trace map, we see that the last two maps in the composites are identical. So we can instead check the equality as maps to $S^{l} \wedge\left|N_{\wedge}^{\text {cy }}(A)\right|$. Now, by the definition of $\sigma$, the composite

$$
\Omega^{l+1}\left(S^{l+1} \wedge G L_{1}(A)\right) \stackrel{\sigma}{\rightarrow} \Omega^{l+1}\left(S^{l} \wedge S_{+}^{1} \wedge G L_{1}(A)\right) \stackrel{p_{1}}{\longrightarrow} \Omega^{l+1}\left(S^{l+1} \wedge G L_{1}(A)\right)
$$

induces the identity map on components. So we can stick this map in between (19) and (20) without changing the map of components. Comparing maps, we then see that it is enough to show that the diagram



commutes. We prove this by constructing a commutative diagram of simplicial sets, which upon realization yields the diagram above. Recall that the forgetful functor from pointed cyclic sets to pointed simplicial sets has a left adjoint. In the notation of [19] it associates to a pointed simplicial set $Y$ the pointed cyclic set $F Y$ with $n$-simplices

$$
F Y[n]=\left\{1, \tau_{n}, \tau_{n}^{2}, \ldots, \tau_{n}^{n}\right\}_{+} \wedge Y[n]
$$

and cyclic structure

$$
\begin{aligned}
d_{i}\left(\tau_{n}^{s}, y\right) & =\left(\tau_{n-1}^{s}, d_{i+s} y\right), & & \text { if } i+s \leq n \\
& =\left(\tau_{n-1}^{s-1}, d_{i+s} y\right), & & \text { if } i+s>n \\
s_{i}\left(\tau_{n}^{s}, y\right) & =\left(\tau_{n+1}^{s}, s_{i+s} y\right), & & \text { if } i+s \leq n \\
& =\left(\tau_{n+1}^{s+1}, s_{i+s} y\right), & & \text { if } i+s>n \\
t_{n}\left(\tau_{n}^{s}, y\right) & =\left(\tau_{n}^{s-1}, y\right), & &
\end{aligned}
$$

where the indices are to be interpreted as their principal representatives modulo $n+1$. The simplices of $F Y$ and $\left(S_{.}^{1}\right)_{+} \wedge Y$ are in one-to-one correspondence, but the simplicial structure is different. However, their realizations are canonically homeomorphic

$$
|F Y| \cong\left|S_{+}^{1} \wedge Y\right|
$$

Moreover, if $X$ is a cyclic set, then the action by $S^{1}$ on the realization is given by the composite

$$
S_{+}^{1} \wedge|X| \stackrel{\text { inv } \wedge|X|}{\longrightarrow} S_{+}^{1} \wedge|X| \cong|F X| \stackrel{\epsilon}{\longrightarrow}|X|
$$


where $\epsilon: F X \rightarrow X$ is the counit. The diagram of simplicial sets we have in mind is the following:

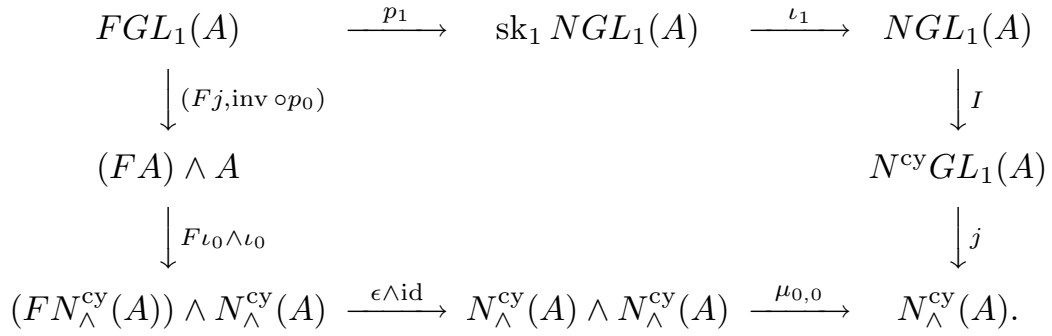

Here the maps $p_{0}$ and $p_{1}$ are simplicial maps given by the formulas

$$
p_{0}\left(\tau_{n}^{s}, x\right)=x, \quad p_{1}\left(\tau_{n}^{s}, x\right)=(1, \ldots, 1, x, 1, \ldots, 1),
$$

where the $x$ is in the $(n-s)$ th spot. Note that these maps are simplicial because $G L_{1}(A)$ is a constant simplicial set. The commutativity of this square is readily checked from the definition of the various maps. The sign in the statement comes from the inversion of the circle in (21).

\section{Global Results}

5.1. Throughout this paragraph, $k$ will be a perfect field of characteristic $p>0$. Let first $X$ a smooth irreducible curve over $k$. Then $\mathrm{TC}_{i}(X ; p)$ vanish for $i \geq 2$, and the group

$$
\mathrm{TC}_{1}(X ; p) \cong K_{1}\left(X, \mathbb{Z}_{p}\right) \cong H_{\text {cont }}^{0}\left(X_{\text {ét }}, \nu \cdot(1)\right)
$$

sits in an exact sequence

$$
0 \rightarrow\left(\mathcal{O}_{X}^{\times}\right)^{\wedge p} \rightarrow \mathrm{TC}_{1}(X ; p) \rightarrow T_{p} \operatorname{Pic} X \rightarrow 0 .
$$

The commutative diagram

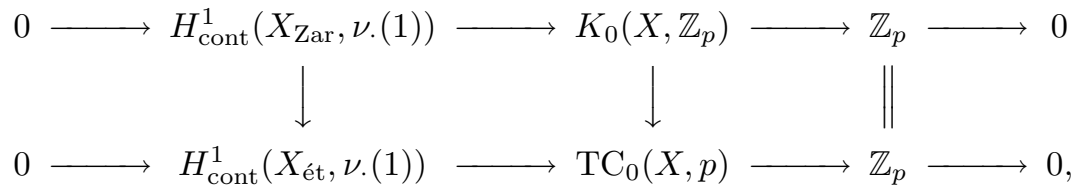

gives a short exact sequence

$$
0 \rightarrow K_{0}\left(X, \mathbb{Z}_{p}\right) \rightarrow \mathrm{TC}_{0}(X ; p) \rightarrow T_{p} \operatorname{Br}(X) \rightarrow 0 .
$$

There is an exact sequence

$$
0 \rightarrow H_{\text {cont }}^{1}\left(X_{\text {Zar }}, R^{1} \epsilon_{*} \nu .(1)\right) \rightarrow \mathrm{TC}_{-1}(X ; p) \rightarrow H_{\text {cont }}^{0}\left(X_{\text {Zar }}, R^{1} \epsilon_{*} \mathbb{Z} / p^{*}\right) \rightarrow 0 .
$$

and finally, $\mathrm{TC}_{-2}(X ; p) \cong H_{\text {cont }}^{1}\left(X_{\mathrm{Zar}}, R^{1} \epsilon_{*} \mathbb{Z} / p^{*}\right)$. In particular, the cyclotomic trace is injective.

We next use the spectral sequence of corollary 4.1.1 to translate two results of Gros-Suwa and Milne into statements about topological cyclic homology. The first result is the following analogue of Parshin's conjecture that higher $K$-groups of smooth projective varieties over finite fields are torsion.

Proposition 5.1.1. Let $X$ be a smooth projective variety over a finite field. Then $\mathrm{TC}_{i}(X ; p)$ is finite except for $i=0$ and $i=-1$. 
Proof. By Gros-Suwa [16, theoreme 4.2.2], the groups $H_{\text {cont }}^{r}\left(X_{\text {ét }}, \nu .(j)\right)$ are finite for $r \neq j, j+1$. The proposition now follows from corollary 4.1.1.

Proposition 5.1.2. Let $X / k$ be a smooth projective variety. Then

a) if $k$ is algebraically closed, $\mathrm{TC}_{i}(X ; p) \otimes \mathbb{Q} \cong \mathrm{TC}_{-i}(X ; p) \otimes \mathbb{Q}$.

b) if $k$ is finite, $\mathrm{TC}_{i}(X ; p) \otimes \mathbb{Q}=\mathrm{TC}_{-i-1}(X ; p) \otimes \mathbb{Q}$.

Proof. By a duality result of Milne, [32, 1.13, 1.14], there are surjections with torsion kernels

and

$$
H_{\text {cont }}^{r}\left(X_{\text {ét }}, \nu \cdot(j)\right) \rightarrow \operatorname{Hom}\left(H_{\text {cont }}^{d-r}\left(X_{\text {ét }}, \nu \cdot(d-j)\right), \mathbb{Z}_{p}\right)
$$

$$
H_{\text {cont }}^{r}\left(X_{\text {ét }}, \nu \cdot(j)\right) \rightarrow \operatorname{Hom}\left(H_{\text {cont }}^{d+1-r}\left(X_{\text {ét }}, \nu \cdot(d-j)\right), \mathbb{Z}_{p}\right),
$$

respectively.

If $X$ is a scheme and $Y$ a smooth closed subscheme of codimension $r$ with open complement $U$, then the purity theorem of Quillen identifies $K(Y)$ with the homotopy fiber of $K(X) \rightarrow K(U)$ yielding the localization sequence

$$
\ldots \rightarrow K_{i}(Y) \rightarrow K_{i}(X) \rightarrow K_{i}(U) \rightarrow \ldots
$$

Let $\operatorname{TC}^{Y}(X ; p)$ be the homotopy fiber of $\mathrm{TC}(X ; p) \rightarrow \mathrm{TC}(U ; p)$ such that one has the long-exact homotopy sequence

$$
\ldots \rightarrow \mathrm{TC}_{i}^{Y}(X ; p) \rightarrow \mathrm{TC}_{i}(X ; p) \rightarrow \mathrm{TC}_{i}(U ; p) \rightarrow \ldots
$$

We note that $\mathrm{TC}^{Y}(X ; p)$ extends to a presheaf of spectra on $X_{\tau}$ which has descent:

$$
\mathrm{TC}^{Y}(V ; p) \stackrel{\sim}{\stackrel{\operatorname{holim}}{\operatorname{hol}}} \mathbb{H}^{\bullet}\left(V, \mathrm{TC}^{Y, \cdot}(-; p)\right) .
$$

Indeed, forming hypercohomology is compatible with cofibration sequences. In general purity fails for topological cyclic homology. However, we have

Proposition 5.1.3. Let $X / k$ be a smooth scheme let and $Y$ be a smooth closed subscheme of dimension e with complement $U$. Then

$$
\mathrm{TC}_{i}^{Y}(X ; p) \cong \mathrm{TC}_{i}(Y ; p)
$$

for $i \geq e$.

Proof. Let $d$ be the dimension of $X, \iota: Y \rightarrow X$ be the closed embedding and let $c=d-e$ the codimension of $Y$ in $X$. Let $\iota^{!}$be the functor which associates to a sheaf the subsheaf of sections with support on $Y$, and let $R^{*} \iota^{!} \nu_{n}(i)$ be the derived functors of $\iota^{!}$. By Milne, [32, prop. 2.1], the derived functors $R^{t} \iota^{\prime} \nu_{n}(i)$ of $\iota^{!}$are trivial unless $t=c$ or $t=c+1$, and $R^{c} \iota^{!} \nu_{n}(i)=\nu_{n}(i-c)$. Hence the spectral sequence

shows that

$$
E_{2}^{s, t}=H_{\text {cont }}^{s}\left(Y_{\text {ét }}, R^{t} \iota^{!} \nu \cdot(i)\right) \Rightarrow H_{Y, \text { cont }}^{s+t}\left(X_{\text {ét }}, \nu \cdot(i)\right)
$$

$$
H_{Y, \text { cont }}^{s}\left(X_{\text {ét }}, \nu \cdot(i)\right)= \begin{cases}H_{\text {cont }}^{0}\left(Y_{\text {ét }}, \nu \cdot(i-c)\right) & \text { if } s=c \\ 0 & \text { if } s<c .\end{cases}
$$

Now consider the descent spectral sequence

$$
E_{2}^{s, t}=H_{Y, \text { cont }}^{s}\left(X_{\text {ét }}, \nu .(-t)\right) \Rightarrow \mathrm{TC}_{-s-t}^{Y}(X ; p) .
$$


Since $H_{Y \text {,cont }}^{s}\left(X_{\text {ét }}, \nu \cdot(-t)\right)=0$ if $-t>d$ or $s<c$, it follows that $\operatorname{TC}_{i}^{Y}(X ; p)=0$ for $i \geq e+1$, and finally,

$$
\mathrm{TC}_{e}^{Y}(X ; p) \cong H_{Y, \text { cont }}^{c}\left(X_{\text {ét }}, \nu .(d)\right) \cong H_{\text {cont }}^{0}\left(Y_{\text {ét }}, \nu \cdot(e)\right) \cong \mathrm{TC}_{e}(Y ; p) .
$$

This proves the claim.

The proposition shows in particular that the restriction

$$
\mathrm{TC}_{i}(X ; p) \stackrel{\sim}{\longrightarrow} \mathrm{TC}_{i}(U ; p)
$$

is an isomorphism for $i \geq e+2$, and that the sequence

$$
0 \rightarrow \mathrm{TC}_{e+1}(X ; p) \rightarrow \mathrm{TC}_{e+1}(U ; p) \rightarrow \mathrm{TC}_{e}(Y ; p) \rightarrow \mathrm{TC}_{e}(X ; p) \rightarrow \mathrm{TC}_{e}(U ; p)
$$

is exact.

5.2. We next consider fields.

Theorem 5.2.1. Let $E^{s}$ be a separable closure of $E$ and let $G_{E}$ be the full Galois group. Then

$$
\mathrm{TC}_{i}(E ; p) \cong K_{i}(E)_{p}^{\wedge} \oplus H_{\mathrm{cont}}^{1}\left(G_{E}, K_{i+1}\left(E^{s}\right)_{p}^{\wedge}\right)
$$

where the second term is the cohomology of the pro-finite group $G_{E}$ acting on the module $K_{i}\left(E^{s}\right)_{p}^{\wedge}$ in the p-adic topology.

Proof. We evaluate the Zariski descent spectral sequence

$$
E_{2}^{s, t}=H_{\text {cont }}^{s}\left((\operatorname{Spec} E)_{\mathrm{Zar}}, \mathcal{T C}_{-t}\right) \Rightarrow \mathrm{TC}_{-s-t}(E ; p)
$$

which by $(13)$ is concentrated on the lines $s=0,1$. Let $\left(\mathcal{K} / p^{*}\right)_{i}$ and $\left(\tilde{\mathcal{K}} / p^{*}\right)_{i}$ denote the sheafifications of the pro-presheaf $K_{i}\left(-, \mathbb{Z} / p^{n}\right)$ on $\mathrm{Sch} / \mathbb{F}_{p}$ for the Zariski and étale topology, respectively. Then theorem 4.2.2 and corollary 4.2 .4 gives an isomorphism of pro-sheaves on $\mathrm{Sm} / \mathbb{F}_{p}$,

$$
\mathcal{T} \mathcal{C}_{i} \cong\left(\mathcal{K} / p^{\cdot}\right)_{i} \oplus R^{1} \epsilon_{*}\left(\tilde{\mathcal{K}} / p^{\cdot}\right)_{i+1}
$$

We claim that this isomorphism is also valid on ( $\operatorname{Spec} E)_{\mathrm{Zar}}$. Indeed, if $E$ is finitely generated, we can find a smooth $\mathbb{F}_{p}$-algebra with function field $E$, and the isomorphism then follows from the one above by taking stalks at the generic point. In general, we write $E$ as the filtered colimit of its finitely generated subfields and use that the functors in question all commute with filtered colimits. For the the left hand side, this uses lemma 2.3.2 and for the second term on the right, [31, III lemma 1.16]. We get an isomorphism on continuous cohomology,

$$
H_{\text {cont }}^{s}\left((\operatorname{Spec} E)_{\mathrm{Zar}}, \mathcal{T} \mathcal{C}_{i}^{\cdot}\right) \cong H_{\text {cont }}^{s}\left((\operatorname{Spec} E)_{\mathrm{Zar}},\left(\mathcal{K} / p^{\cdot}\right)_{i} \oplus R^{1} \epsilon_{*}\left(\tilde{\mathcal{K}} / p^{\cdot}\right)_{i+1}\right) .
$$

Now by definition,

$$
H^{0}\left((\operatorname{Spec} E)_{\mathrm{Zar}},\left(\mathcal{K} / p^{n}\right)_{i}\right)=K_{i}\left(E, \mathbb{Z} / p^{n}\right),
$$

and since $K_{i}(E)$ has no $p$-torsion, this is isomorphic to $K_{i}(E) / p^{n}$. Hence the short exact sequence for continuous cohomology (13) shows that

$$
H_{\text {cont }}^{s}\left((\operatorname{Spec} E)_{\mathrm{Zar}},\left(\mathcal{K} / p^{\cdot}\right)_{i}\right) \cong \begin{cases}K_{i}(E)_{p}^{\wedge} & \text { if } s=0 \\ 0 & \text { if } s=1\end{cases}
$$


The vanishing of the group in dimension 1 and the change of topology spectral sequence then yields an isomorphism

$$
H_{\text {cont }}^{0}\left((\operatorname{Spec} E)_{\text {Zar }}, R^{1} \epsilon_{*}\left(\tilde{\mathcal{K}} / p^{\cdot}\right)_{i+1}\right) \cong H_{\text {cont }}^{1}\left((\operatorname{Spec} E)_{\text {ét }},\left(\tilde{\mathcal{K}} / p^{*}\right)_{i+1}\right),
$$

and since $K$-theory commutes with filtered colimits, the right hand group is isomorphic to $H_{\text {cont }}^{1}\left(G_{E}, K_{i+1}\left(E^{s}\right)_{p}^{\wedge}\right)$ by $[25$, theorem 2.2]. Finally, we again use the change of topology spectral sequence, and the fact that the 2-dimensional continuous Zariski cohomology of a field vanishes, to conclude that

$$
H_{\text {cont }}^{1}\left((\operatorname{Spec} E)_{\text {Zar }}, R^{1} \epsilon_{*}\left(\tilde{\mathcal{K}} / p^{*}\right)_{i+1}\right) \cong H_{\text {cont }}^{2}\left((\operatorname{Spec} E)_{\text {ét }},\left(\tilde{\mathcal{K}} / p^{*}\right)_{i+1}\right) .
$$

But this group is trivial as one sees by writing $E$ as a filtered colimit of smooth $\mathbb{F}_{p^{-}}$-algebra and using theorem 4.2.2 and lemma 4.1.2.

In the lowest two dimensions we get

$$
\begin{aligned}
& \operatorname{TC}_{0}(E ; p) \cong \mathbb{Z}_{p} \oplus T_{p} \operatorname{Br}(E) \\
& \mathrm{TC}_{-1}(E ; p) \cong \operatorname{Hom}\left(\operatorname{Gal}(E), \mathbb{Z}_{p}\right)
\end{aligned}
$$

The proof of theorem 5.2.1 works more generally for filtered colimits of essentially smooth local algebras over a perfect field. For an essentially smooth local $k$-algebra of dimension $d$, we get

$$
\mathrm{TC}_{i}(A ; p)= \begin{cases}0 & \text { if } i>d \\ K_{d}(A)_{p}^{\wedge} & \text { if } i=d .\end{cases}
$$

If $k$ is algebraically closed, then in addition

$$
\mathrm{TC}_{d-1}(A ; p) \cong K_{d-1}(A)_{p}^{\wedge},
$$

because $R^{1} \epsilon_{*} \nu .(d)=0$ if $B$ is over an algebraically closed field, [16, lemme III 3.16].

We recall that a field $E$ has property $C_{j}$ if every polynomial $f \in E\left[x_{1}, \ldots, x_{n}\right]$ of degree $d$ with $n>d^{j}$ has a nontrivial zero in $E^{n}$. For example, a field is $C_{0}$ if and only if it is separably closed, and a field with property $C_{j}$ has property $C_{l}$ for all $l \geq j$. The generalized Tsen theorem [15, Theorem 3.6] states that for $L / E$ an extension of transcendence degree $l$ and $E$ a $C_{j}$-field, the field $L$ is $C_{j+l}$.

Corollary 5.2.2. Let $E$ be a field of characteristic $p$ with property $C_{j}$. Then the cyclotomic trace

$$
\operatorname{trc}: K_{i}\left(E, \mathbb{Z}_{p}\right) \rightarrow \mathrm{TC}_{i}(E ; p)
$$

is an isomorphism for $i \geq j$.

Proof. We follow [35, Satz 3.1.3]. The exact sequence

$$
K_{j}\left(E^{s}\right) / p \rightarrow K_{j}\left(E^{s}\right) / p^{n} \rightarrow K_{j}\left(E^{s}\right) / p^{n-1} \rightarrow 0,
$$

and the fact that $K_{j}\left(E^{s}\right) / p \cong \nu_{1}(j)\left(E^{s}\right)$ for any field $E$, shows that it suffices to prove $H^{1}\left(G_{E}, \nu_{1}(j)\left(E^{s}\right)\right)=0$. There is a short exact sequence

$$
0 \rightarrow \nu_{1}(j)\left(E^{s}\right) \rightarrow \Omega_{E^{s}}^{j} \stackrel{C^{-1}-1}{\longrightarrow} \Omega_{E^{s}}^{j} / d \Omega_{E^{s}}^{j-1} \rightarrow 0,
$$

where $C^{-1}$ is the inverse of the Cartier operator, and since the the higher cohomology of a quasi-coherent module vanishes, we see that this group is isomophic to the cokernel of the map $C^{-1}-1: \Omega_{E}^{i} \rightarrow \Omega_{E}^{i} / d \Omega_{E}^{i-1}$. But [28, Prop. $\left.2(2)\right]$ shows that this map is onto. 
5.3. Let $A$ be complete discrete valuation ring with fraction field $E$ and perfect residue field $k$ of equal characteristic $p$. If $t$ is a uniformizer, then $A \cong k[[t]]$ and $E \cong k((t))$. Generalizing a theorem of C. Moore, B. Kahn, [26], has shown that

$$
K_{2}(E) \cong K_{2}(k) \oplus k^{\times} \oplus D,
$$

where $D$ is divisible group which according to [39] is $p$-torsion free. In follows that $K_{i}^{M}(E)_{p}^{\wedge}=0$, for $i \geq 2$, and the theorem of the first author and Levine, 4.2.1, then shows that so is $K_{i}\left(E, \mathbb{Z}_{p}\right)$. Since the $p$-adic $K$-theory of a perfect field is equal to $\mathbb{Z}_{p}$ concentrated in degree zero, the localization sequence gives:

$$
K_{i}\left(A, \mathbb{Z}_{p}\right) \cong \begin{cases}\mathbb{Z}_{p} & \text { if } i=0 \\ (1+t A)^{\times} & \text {if } i=1 \\ 0 & \text { else. }\end{cases}
$$

We compare this to the continuous $K$-theory of $A$ defined by

$$
K^{c}(A)=\underset{n}{\operatorname{holim}} K\left(A / \mathfrak{m}^{n}\right)
$$

Using a theorem of McCarthy, the relative term $K_{*}^{c}(A, \mathfrak{m})$ was evaluated in [18, theorem $\mathrm{C}]$ and shown to be equal to $(1+t A)^{\times}$concentrated in dimension one. Combining these results, we get

Proposition 5.3.1. Let $A$ be a complete discrete valuation ring with perfect residue field of equal characteristic $p>0$. Then the canonical map

$$
K_{i}\left(A, \mathbb{Z}_{p}\right) \stackrel{\sim}{\longrightarrow} K_{i}^{c}\left(A, \mathbb{Z}_{p}\right)
$$

is an isomorphism.

The corresponding statement in the unequal characteristic case was proved earlier by Suslin and Panin, [36]. Let us also note in that in either case, a theorem of Gabber, [9], shows that for $l$-adic $K$-theory, both of the maps

$$
K_{i}\left(A, \mathbb{Z}_{l}\right) \stackrel{\sim}{\longrightarrow} K_{i}^{c}\left(A, \mathbb{Z}_{l}\right) \stackrel{\sim}{\longrightarrow} K_{i}\left(k, \mathbb{Z}_{l}\right)
$$

are isomorphism.

We also use theorem 5.2.1 to evaluate the topological cyclic homology of $E$. The group $H_{\text {cont }}^{1}\left(G_{E}, K_{1}\left(E^{s}\right)_{p}^{\wedge}\right)$ is isomorphic to the Tate module of the Brauer group, and we recall from $[38$, p. 186$]$ that

$$
T_{p} \operatorname{Br}(E) \cong T_{p} \operatorname{Br}(k) \oplus \operatorname{Hom}\left(G_{k}, \mathbb{Z}_{p}\right),
$$

and form op. cit. p. 157 that $\operatorname{Br}(k)$ is $p$-torsion free such that the first term on the right vanishes. Thus

$$
\mathrm{TC}_{i}(E ; p) \cong \begin{cases}\operatorname{Hom}\left(G_{E}, \mathbb{Z}_{p}\right) & \text { if } i=-1 \\ \mathbb{Z}_{p} \oplus \operatorname{Hom}\left(G_{k}, \mathbb{Z}_{p}\right) & \text { if } i=0 \\ (1+t A)^{\times} \oplus \mathbb{Z}_{p} & \text { if } i=1 \\ 0 & \text { if } i \geq 2\end{cases}
$$

We can also evaluate the descent sequence

$$
E_{2}^{s, t}=H_{\text {cont }}^{s}\left(X_{\text {ét }},\left(\mathcal{K} / p^{*}\right)_{-t}\right) \Rightarrow \pi_{-s-t}\left(\mathbb{H}^{*}\left(X_{\text {ét }}, K(-)\right)_{p}^{\wedge}\right),
$$


where $X=\operatorname{Spec} A$. Indeed, an étale extension of a power series ring again is a power series ring, and hence the vanishing of $K_{i}\left(A, \mathbb{Z}_{p}\right)$ for $i>1$ implies that the sheaves $\left(\mathcal{K} / p^{*}\right)_{i}$ on $X_{\text {ét }}$ vanish for $i>1$. Thus we have

$$
\pi_{i}\left(\mathbb { H } ^ { \cdot } ( X _ { \text { ét } } , K ( - ) ) _ { p } ^ { \wedge } \cong \left\{\begin{array}{ll}
H_{\text {cont }}^{1}\left(X_{\text {ét }}, \mathbb{Z} / p^{*}\right) \cong \operatorname{Hom}\left(G_{k}, \mathbb{Z}_{p}\right) & \text { if } i=-1 \\
H_{\text {cont }}^{0}\left(X_{\text {ét }}, \mathbb{Z} / p^{*}\right) \cong \mathbb{Z}_{p} & \text { if } i=0 \\
H_{\text {cont }}^{0}\left(X_{\text {ét }},\left(\mathcal{K} / p^{*}\right)_{1}\right) \cong(1+t A)^{\times} & \text {if } i=1 \\
0 & \text { otherwise }
\end{array}\right.\right.
$$

For $i=-1$, this follows from [31, Prop.I 4.4, III 4]. For $i=0$, the group $H^{1}\left((\operatorname{Spec} A)_{\text {ét }},\left(\mathcal{K} / p^{*}\right)_{1}\right)$, vanishes because the Brauer group of a Henselian local ring injects into the Brauer group of its residue field [31, Prop. IV 1.6]. Finally, for $i=1$ we use the long exact sequence to the short exact sequence

$$
0 \rightarrow \mathbb{G}_{m} \stackrel{p^{n}}{\longrightarrow} \mathbb{G}_{m} \rightarrow \mathcal{K}_{1} / p^{n} \rightarrow 0
$$

and the fact that the Picard group of a local ring vanishes. This calculation shows that

$$
\operatorname{TC}^{c}(A, p) \simeq \mathbb{H}^{\cdot}\left((\operatorname{Spec} A)_{\text {ét }}, K / p^{*}\right)_{p}^{\wedge},
$$

since both spectra are products of Eilenberg-MacLane spectra. We would like to use corollary 4.2.6 to conclude that $\mathrm{TC}(A ; p) \simeq \operatorname{TC}^{c}(A ; p)$ thereby answering [20, Remark 6.2]. But $A$ is only a regular local ring and not an essentially smooth $k$-algebra as required. It seems reasonable to expect, however, that corollaries 4.2.5 and 4.2.6 are valid more generally for regular $k$-algebras. Note that continuity in this situation also is implied by and implies short exact localization sequences

$$
0 \rightarrow \mathrm{TC}_{1}(A ; p) \rightarrow \mathrm{TC}_{1}(E ; p) \rightarrow \mathrm{TC}_{0}(k ; p) \rightarrow 0
$$

and

$$
0 \rightarrow \mathrm{TC}_{0}(A ; p) \rightarrow \mathrm{TC}_{0}(E ; p) \rightarrow \mathrm{TC}_{-1}(k ; p) \rightarrow 0,
$$

analogous to the Gersten resolution for $K$-theory. Indeed, the groups $\mathrm{TC}_{*}^{c}(A ; p)$ fit in such a sequences.

5.4. This section concerns the Zariski descent spectral sequence for topological cyclic homology. First, we briefly recall the construction of this sequence and refer the reader to [7] for details.

The descent spectral sequence is a particular case of the spectral sequence associated to the homotopy limit of a diagram $\mathcal{X}$ of quasi-fibrant symmetric spectra,

$$
E_{2}^{s, t}={\underset{I}{\lim }}^{s} \pi_{-t} \mathcal{X} \Rightarrow \pi_{-s-t} \underset{I}{\operatorname{holim}} \mathcal{X}
$$

By definition, the homotopy limit is the total spectrum of the cosimplicial replacement $\Pi_{I} \mathcal{X}$ of the diagram. The coskeleton filtration of the total spectrum gives a natural tower fibrations $\left\{F^{s} \mathcal{X}\right\}_{s \geq 0}$ with inverse limit holim $\mathcal{X}$, and the spectral sequence is then given by the exact couple

$$
\begin{aligned}
D^{s, t} & =\pi_{-s-t} F^{s} \mathcal{X} \\
E^{s, t} & =\pi_{-s-t} \operatorname{fiber}\left(F^{s} \mathcal{X} \rightarrow F^{s-1} \mathcal{X}\right) .
\end{aligned}
$$


The homotopy groups $\pi_{-t} \mathcal{X}$ form a diagram of abelian groups and it follows immediately from the definitions that

$$
E^{s, t} \cong N \Pi_{I}^{s} \pi_{-t} \mathcal{X}=\operatorname{ker} s^{0} \cap \cdots \cap \operatorname{ker} s^{s-1} \subset \Pi_{I}^{s} \pi_{-t} \mathcal{X}
$$

with differential $d=\sum(-1)^{i} d^{i}$. This is the normalized complex associated with the cosimplicial replacement of the diagram $\pi_{-t} \mathcal{X}$ of abelian groups. Now recall from $\left[7\right.$, XI 6.2] that for any diagram $\mathcal{A}$ of abelian groups, the cochain complex $\Pi_{I}^{*} \mathcal{A}$ associated with the cosimplicial abelian group $\Pi_{I} \mathcal{A}$ and the normalized subcomplex $N \Pi_{I}^{*} \mathcal{A}$ both calculate the higher derived limits of $\mathcal{A}$,

$$
\varliminf_{I} \lim ^{s} \mathcal{A} \cong H^{s}\left(\Pi_{I}^{*} \mathcal{A}\right) \cong H^{s}\left(N \Pi_{I}^{*} \mathcal{A}\right) \text {. }
$$

Hence the stated $E_{2}$-term.

We also recall the change of topology spectral sequence

$$
E_{2}^{i, j}=H_{\text {cont }}^{i}\left(X_{\mathrm{Zar}}, R^{j} \epsilon_{*} F .\right) \Rightarrow H_{\text {cont }}^{i+j}\left(X_{\text {ét }}, F .\right),
$$

which by definition is the Grothendieck spectral sequence given by the composition of functors $\lim \Gamma(X,-) \circ \epsilon_{*}$. To construct it, one first chooses an injective resolution $C^{*}$. of the étale pro-sheaf $F$. and then a fully injective resolution $I^{*, *}$ of the complex $\epsilon_{*} C_{\text {. }}^{*}$. Recall that in addition to $I^{*, *}$ being a double complex such that $I^{*, j}$ is an injective resolution of $\epsilon_{*} C^{j}$, for all $j \geq 0$, this means that the complexes

$$
\begin{aligned}
{ }^{2} Z^{*, j} & =\operatorname{ker}\left(I^{*, j} \rightarrow I_{.}^{*, j+1}\right) \\
{ }^{2} B^{*, j} & =\operatorname{im}\left(I^{*, j-1} \rightarrow I^{*, j}\right) \\
{ }^{2} H_{.}^{*, j} & =\operatorname{coker}\left({ }^{2} B^{*, j} \longrightarrow{ }^{2} Z^{*, j}\right)
\end{aligned}
$$

are injective resolutions of $Z^{j}\left(C^{*}\right), B^{j}\left(C_{.}^{*}\right)$ and $H^{j}\left(C_{.}^{*}\right)$, respectively. The spectral sequence is then given by the double complex

$$
\varliminf_{\mathbb{N}} \Gamma\left(X, I^{*, *}\right)
$$

Lemma 5.4.1. Let $F$. be an étale pro-sheaf and let $C^{*}$ be an $\epsilon_{*}$-acyclic resolution of $F$. such that for all $j \geq 0, \epsilon_{*} C^{j}$. is $\lim \Gamma(X,-)$-acyclic. Then the double complex

$$
N \Pi_{\Delta \times \mathbb{N}}^{*} \Gamma\left(X, G^{*} \epsilon_{*} C^{*}\right)
$$

gives rise to the change of topology spectral sequence.

Proof. One can always replace an injective resolution of $F$. with a resolution $C$. with the stated properties is standard. So let $I^{*, *}$ be a fully injective resolution of $\epsilon_{*} C^{*}$. We can assume that each of $I^{*, j}$ is the cochain complex associated with a cosimplicial pro-sheaf $I^{*}, j$ and that $I^{*, j} \rightarrow I^{*, j+1}$ is induced from a cosimplicial map. Indeed, the categories of cochain complexes and cosimplicial objects in an abelian category are canonically equivalent. We now construct maps of double complexes

$$
\varliminf_{\mathbb{N}}^{\lim _{N}} \Gamma\left(X, I^{*, *}\right) \rightarrow \Pi_{\Delta \times \mathbb{N}}^{*} \Gamma\left(X, I^{*^{*}}\right) \leftarrow N \Pi_{\Delta \times \mathbb{N}}^{*} \Gamma\left(X, I^{\cdot *}{ }^{*}\right)
$$

such that the induced maps of $E_{2}$-terms are isomorphisms. To this end, first note that for any injective pro-sheaf $J$. , we have a quasi-isomorphism

$$
\varliminf_{\mathbb{N}}^{\lim } \Gamma\left(X, J_{.}\right) \stackrel{\sim}{\longrightarrow} \Pi_{\mathbb{N}}^{*} \Gamma\left(X, J_{.}\right) \text {. }
$$


Indeed, this follows form (22) and the fact that the structure maps in $J$. are split epimorphisms. Next, recall from [7, XI 7.3] that for any cosimplicial abelian group $A^{*}$, there is a canonical quasi-isomorphism

$$
A^{*} \stackrel{\sim}{\longrightarrow} \Pi_{\Delta}^{*} A^{*}
$$

Combining these quasi-isomorphisms, we obtain that for any cosimplicial injective pro-sheaf $J \cdot$, there is a canonical quasi-isomorphism

$$
\varliminf_{\mathbb{N}} \Gamma\left(X, J_{.}^{*}\right) \stackrel{\sim}{\longrightarrow} \Pi_{\Delta}^{*}\left(\Pi_{\mathbb{N}}^{*} \Gamma\left(X, J_{*}^{*}\right)\right) \cong \Pi_{\Delta \times \mathbb{N}}^{*} \Gamma\left(X, J_{*}^{*}\right)
$$

and finally, the inclusion of the normalized cochain complex

$$
\Pi_{\Delta \times \mathbb{N}}^{*} \Gamma\left(X, J^{*}\right) \stackrel{\sim}{\longleftarrow} \Pi_{\Delta \times \mathbb{N}}^{*} \Gamma\left(X, J_{:}^{*}\right)
$$

is a quasi-isomorphism. This establishes the maps in (23) and shows that we have quasi-isomorphisms

$$
\varliminf_{\mathbb{N}} \Gamma\left(X,{ }^{2} H^{*, j}\right) \stackrel{\sim}{\longrightarrow} \Pi_{\Delta \times \mathbb{N}}^{*} \Gamma\left(X,{ }^{2} H^{,}, j\right) \stackrel{\sim}{\longleftarrow} N \Pi_{\Delta \times \mathbb{N}}^{*} \Gamma\left(X,{ }^{2} H^{\cdot}, j\right),
$$

and hence the induced maps on $E_{2}$ are isomorphisms.

To prove the lemma, we now use that $I .^{* *}$ is injective to get a map of cosimplicial cochain-complexes of pro-sheaves

$$
G^{\cdot} \epsilon_{*} C^{*} \rightarrow I^{* * *}
$$

lifting the identity on $\epsilon_{*} C^{*}$. We can do this because the categories of cochain complexes and cosimplicial objects in an abelian category are canonically equivalent. The induced map of double complexes

$$
N \Pi_{\Delta \times \mathbb{N}}^{*} \Gamma\left(X, G^{*} \epsilon_{*} C^{*}\right) \rightarrow N \Pi_{\Delta \times \mathbb{N}}^{*} \Gamma\left(X, I^{*}{ }^{*}\right)
$$

induces an isomorphism of $E_{2}$-terms. For the fact that $G^{\cdot}$ is an exact functor together with (22) and lemma 3.1.3 shows that the induced map of $E_{1}$-terms is a horizontal quasi-isomorphism.

We recall from lemma 4.1.2 that $\nu .(j)$ has only a single derived image, such that the change of topology spectral sequence becomes a long-exact sequence.

Proposition 5.4.2. Let $X / k$ be a smooth affine scheme and let $E_{r}^{s, t}$ be the Zariski descent sequence for topological cyclic homology. Then the composite map

$$
H_{\text {cont }}^{s}\left(X_{\mathrm{Zar}}, R^{1} \epsilon_{*} \nu \cdot(-t+1)\right) \longmapsto E_{2}^{s, t} \stackrel{d_{2}}{\longrightarrow} E_{2}^{s+2, t-1} \rightarrow H^{s+2}\left(X_{\mathrm{Zar}}, \nu \cdot(-t+1)\right)
$$

has the same kernel and image as the boundary map in the change of topology long-exact sequence.

Proof. Let $\mathcal{X}$ and ' $\mathcal{X}$ denote the diagrams

$$
\begin{aligned}
\mathcal{X} & =\Gamma\left(X,\left|G^{\cdot} \operatorname{Sin} \mathrm{TC}^{\cdot}(-; p)\right|\right) \\
{ }^{\prime} \mathcal{X} & =\Gamma\left(X,\left|G^{\cdot} \operatorname{Sin} \operatorname{TR}^{\cdot}(-; p)\right|\right),
\end{aligned}
$$

where the Thomason-Godement construction is formed with respect to the Zariski topology on $X$. The homotopy limits are equal to $\operatorname{TC}\left(X_{\text {Zar }} ; p\right)$ and $\operatorname{TR}\left(X_{\text {Zar }} ; p\right)$, respectively, and we write $\left(E^{s, t}, D^{s, t}\right)$ and $\left({ }^{\prime} E^{s, t},{ }^{\prime} D^{s, t}\right)$ for the corresponding exact couples. The maps of diagrams

$$
\mathcal{X} \rightarrow{ }^{\prime} \mathcal{X} \underset{34}{\stackrel{1-F}{\longrightarrow}} \text { 'X } \rightarrow \Sigma \mathcal{X}
$$


induce maps of exact couples - and hence of spectral sequences - and the sequences

$$
\begin{aligned}
& \ldots \stackrel{\delta}{\longrightarrow} E^{s, t} \stackrel{\iota}{\rightarrow} E^{s, t} \stackrel{1-F}{\longrightarrow}{ }^{s, t} \stackrel{\delta}{\longrightarrow} E^{s, t-1} \stackrel{\iota}{\longrightarrow} \ldots \\
& \ldots \stackrel{\delta}{\longrightarrow} D^{s, t} \stackrel{\iota}{\longrightarrow} D^{s, t} \stackrel{1-F}{\longrightarrow} D^{s, t} \stackrel{\delta}{\longrightarrow} D^{s, t-1} \stackrel{\iota}{\longrightarrow} \ldots
\end{aligned}
$$

are exact. Indeed, these are the long exact sequences in homotopy induced from a cofibration sequence of spectra.

We let $C^{*}$. be the complex of étale pro-sheaves

$$
\mathcal{T R}_{-t} \stackrel{1-F}{\longrightarrow} \mathcal{T} \mathcal{R}_{-t}^{\cdot}
$$

This is a resolution of $\nu$. $(-t)$ by (15) which is $\epsilon_{*}$-acyclic by corollary 4.1.1. And since $X$ is affine, lemma 4.1.2 shows that the pro-sheaves $\epsilon_{*} C^{j}$. are $\lim _{\longleftarrow} \Gamma(X,-)$-acyclic. By the previous lemma, we therefore conclude that the double complex

$$
N \Pi_{\Delta \times \mathbb{N}}^{*} \Gamma\left(X, G^{*} \epsilon_{*} C^{*}\right)
$$

defines the change of topology spectral sequence. But written out, this double complex is

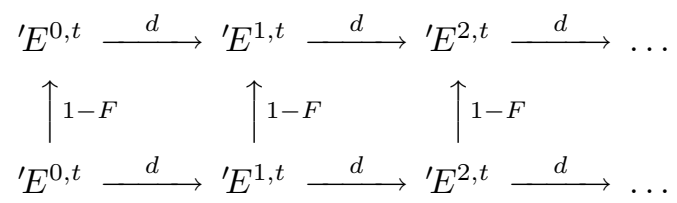

and hence the boundary map $\partial$ in the change of topology long-exact sequence is given by the additive relation

$$
{ }^{\prime} E^{s, t-1} \stackrel{d}{\longrightarrow} E^{s+1, t-1} \stackrel{1-F}{\longleftarrow}{ }^{s} E^{s+1, t-1} \stackrel{d}{\longrightarrow}{ }^{\prime} E^{s+2, t-1} .
$$

The reader is referred to [29] for the notion of additive relations. We want to compare this to the $d_{2}$-differential in the Zariski descent sequence which is given by the additive relation

$$
E^{s, t} \stackrel{j}{\rightarrow} D^{s, t} \stackrel{i}{\leftarrow} D^{s+1, t-1} \stackrel{k}{\rightarrow} E^{s+2, t-1} .
$$

It follows from (25) and (24) that an element of $x \in E^{s, t}$ represents a class in

$$
H_{\text {cont }}^{s}\left(X_{\text {Zar }}, R^{1} \epsilon_{*} \nu \cdot(-t+1)\right) \subset E_{2}^{s, t}
$$

if and only if $j x \in D^{s, t}$ lies in the image of $\delta \circ j:{ }^{\prime} E^{s, t-1} \rightarrow D^{s, t}$. It also follows that the image of a class $d_{2} x$ in the quotient

$$
E_{2}^{s+2, t-1} \rightarrow H_{\text {cont }}^{s+2}\left(X_{\text {Zar }}, \nu \cdot(-t+1)\right)
$$

is equal to the image of a representative under the map $\iota: E^{s+1, t-1} \rightarrow{ }^{\prime} E^{s+1, t-1}$ modulo the image of the map $\iota \circ d: E^{s+1, t-1} \rightarrow{ }^{\prime} E^{s+1, t-1}$. In other words, the composite map of the statement is given by the composite additive relation

$$
{ }^{\prime} E^{s, t-1} \stackrel{j}{\longrightarrow} D^{s, t-1} \stackrel{\delta}{\longrightarrow} D^{s, t} \stackrel{i}{\leftarrow} D^{s+1, t-1} \stackrel{\iota}{\longrightarrow} D^{s+1, t-1} \stackrel{k}{\longrightarrow} E^{s+2, t-1} .
$$

On the other hand, the boundary map $\partial$, we recall, is given by the additive relation

$$
{ }^{\prime} E^{s, t-1} \stackrel{j}{\longrightarrow} D^{s, t-1} \stackrel{k}{\longrightarrow} E^{s+1, t-1} \stackrel{1-F}{\longleftarrow} E^{s+1, t-1} \stackrel{j}{\longrightarrow} D^{s+1, t-1} \stackrel{k}{\longrightarrow}{ }^{\prime} E^{s+2, t-1} .
$$


The two additive relations have the their first and last map in common so we may compare the additive relations

$$
\begin{aligned}
& \theta_{1}:{ }^{\prime} D^{s, t-1} \stackrel{\delta}{\longrightarrow} D^{s, t} \stackrel{i}{\longleftarrow} D^{s+1, t-1} \stackrel{\iota}{\longrightarrow} D^{s+1, t-1}, \\
& \theta_{2}:{ }^{\prime} D^{s, t-1} \stackrel{k}{\longrightarrow} E^{s+1, t-1} \stackrel{1-F}{\longleftarrow} E^{s+1, t-1} \stackrel{j}{\longrightarrow} D^{s+1, t-1}
\end{aligned}
$$

Chasing through the diagram

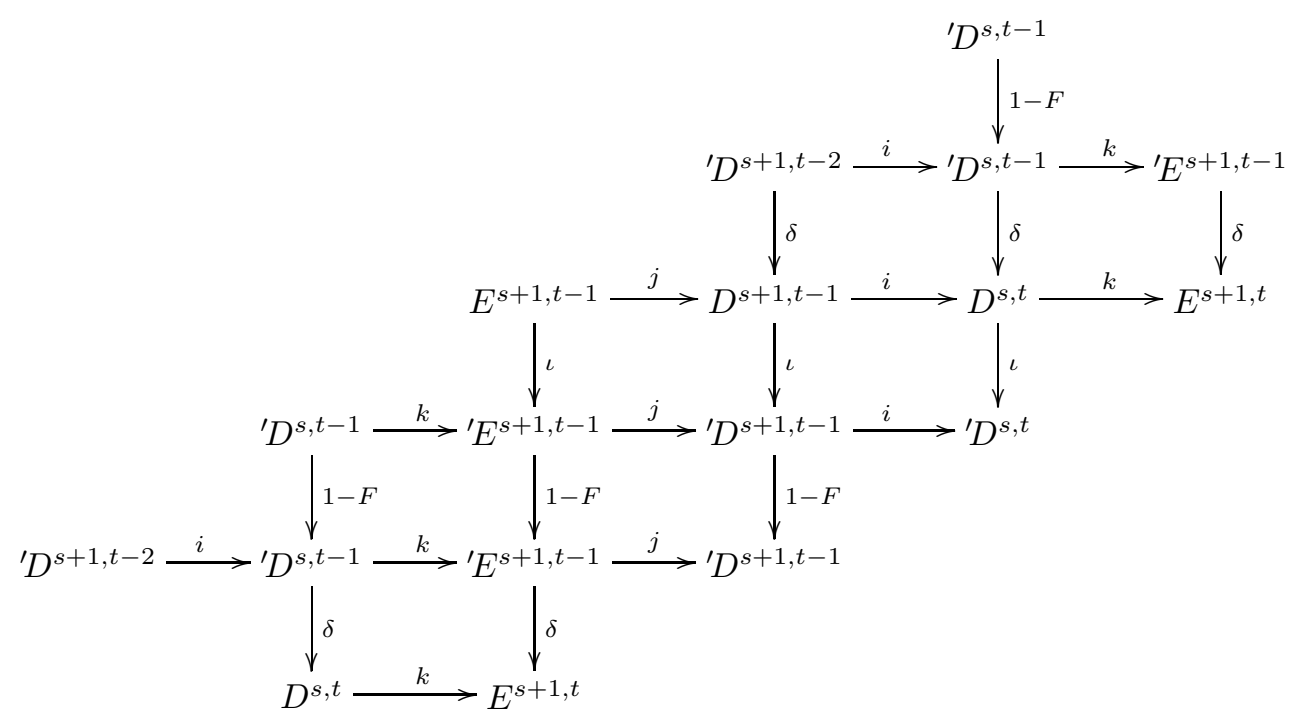

in which all rows and columns are exact, we see that in both cases

$$
\begin{aligned}
\operatorname{def} \theta_{n} & =\operatorname{ker}(\delta \circ k) \\
\operatorname{im} \theta_{n} & =\operatorname{ker} i \cap \operatorname{ker}(1-F) \\
\operatorname{ker} \theta_{n} & =\operatorname{im} i+\operatorname{im}(1-F) \\
\operatorname{ind} \theta_{n} & =\operatorname{im}(\iota \circ j) .
\end{aligned}
$$

Hence the domain and target of the induced isomorphisms

$$
\theta_{n}: \operatorname{def} \theta_{n} / \operatorname{ker} \theta_{n} \stackrel{\sim}{\longrightarrow} \operatorname{im} \theta_{n} / \operatorname{ind} \theta_{n}
$$

are independent of $n$ albeit the isomorphisms may differ. At any rate, it follows that the kernels and the images of the maps $\partial$ and $\partial^{\prime}$ are equal. This concludes the proof.

Corollary 5.4.3. The Zariski descent sequence for topology cyclic homology of a smooth affine scheme $X / k$ degenerates at $E_{3}$, with the $E_{3}$-term concentrated on the lines $s=0,1$. Moreover, the map $\iota$ induces an isomorphism

$$
E_{3}^{1, t} \cong H_{\mathrm{cont}}^{1}\left(X_{\mathrm{Zar}}, \nu \cdot(-t)\right)
$$

and there is a natural exact sequence

$$
H_{\text {cont }}^{1}\left(X_{\text {Zar }}, \nu \cdot(-t+1)\right) \longmapsto H_{\text {cont }}^{1}\left(X_{\text {ét }}, \nu \cdot(-t+1)\right) \rightarrow E_{3}^{0, t} \rightarrow H_{\text {cont }}^{0}\left(X_{\text {Zar }}, \nu \cdot(-t)\right),
$$

where the left hand map is the change of topology homomorphism. 
Proof. As $X$ is smooth affine, lemma 4.1.2 shows that the boundary map in the change of topology exact sequence

$$
\partial: H_{\text {cont }}^{s}\left(X_{\text {Zar }}, R^{1} \epsilon_{*} \nu \cdot(-t+1)\right) \rightarrow H_{\text {cont }}^{s+2}\left(X_{\text {Zar }}, \nu \cdot(-t+1)\right)
$$

is an isomorphism, for $s \geq 1$, and that one has an exact sequence

$$
\begin{aligned}
0 \rightarrow H_{\text {cont }}^{1}\left(X_{\text {Zar }}, \nu \cdot(-t+1)\right) \rightarrow H_{\text {cont }}^{1}\left(X_{\text {ét }}, \nu \cdot(-t+1)\right) \\
\quad \rightarrow H_{\text {cont }}^{0}\left(X_{\text {Zar }}, R^{1} \epsilon_{*} \nu \cdot(-t+1)\right) \stackrel{\partial}{\rightarrow} H_{\text {cont }}^{2}\left(X_{\text {Zar }}, \nu \cdot(-t+1)\right) \rightarrow 0 .
\end{aligned}
$$

Now let $s \geq 2$ and consider the exact sequence

$$
0 \rightarrow H_{\text {cont }}^{s}\left(X_{\text {Zar }}, R^{1} \epsilon_{*} \nu .(-t+1)\right) \stackrel{\delta}{\rightarrow} E_{2}^{s, t} \stackrel{\iota}{\rightarrow} H_{\text {cont }}^{s}\left(X_{\text {Zar }}, \nu \cdot(-t)\right) \rightarrow 0 .
$$

The lemma and the preceding remarks shows that $d_{2}^{s, t}: E_{2}^{s, t} \rightarrow E_{2}^{s+2, t-1}$ is nontrivial on the image of the map $\delta$, and hence $\iota$ maps the $d_{2}$-cycles in $E_{2}^{s, t}$ into the right hand group in the sequence above. By the same reasoning, $\iota$ also maps the $d_{2}$-boundaries in $E_{2}^{s, t}$ onto the right hand group. Hence the subgroups of cycles and boundaries in $E_{2}^{s, t}$ are equal and isomorphic to the right hand group, the isomorphism given by $\iota$.

When $s=1$, the a similar argument shows that the image of $\delta$ is mapped isomorphically onto the subgroup of boundaries in $E_{2}^{3, t}$. Therefore, $\iota$ maps the subgroup of cycles in $E_{2}^{1, t}$ is isomorphically onto the right hand group in the sequence above, and hence $E_{3}^{1, t}$ is as claimed. Finally, this argument applied to the sequence

$$
\begin{aligned}
0 \rightarrow H_{\mathrm{cont}}^{0}\left(X_{\mathrm{Zar}}, R^{1} \epsilon_{*} \nu \cdot(-t+1)\right) & / \operatorname{ker} \partial \\
& \stackrel{\delta}{\rightarrow} E_{2}^{0, t} / \delta(\operatorname{ker} \partial) \stackrel{\iota}{\rightarrow} H_{\mathrm{cont}}^{0}\left(X_{\mathrm{Zar}}, \nu \cdot(-t)\right) \rightarrow 0
\end{aligned}
$$

shows that the quotient of the cycles in $E_{2}^{0, t}$ by the image of ker $\partial$ is mapped isomorphically onto the right hand group by $\iota$. This gives the stated short exact sequence for $E_{3}^{0, t}$.

In comparison, the étale descent sequence for topological cyclic homology of a smooth affine scheme collapses at $E_{2}$. Indeed, lemma 4.1.2 shows that the $E_{2}$-term is concentrated on the lines $s=0,1$. Hence this spectral sequence amounts to short exact sequences

$$
0 \rightarrow H_{\text {cont }}^{1}\left(X_{\text {ét }}, \nu \cdot(-t+1)\right) \rightarrow \mathrm{TC}_{i}(X ; p) \rightarrow H_{\text {cont }}^{0}\left(X_{\text {ét }}, \nu \cdot(-t)\right) \rightarrow 0 .
$$

We can compare this filtration to the filtration induced from the Zariski descent sequence. For the change of topology map

$$
\mathrm{TC}\left(X_{\text {Zar }} ; p\right) \stackrel{\sim}{\longrightarrow} \mathrm{TC}\left(X_{\text {ét }} ; p\right),
$$

which we remember is a weak equivalence, induces a map from the Zariski descent sequence to the étale descent sequence and hence a map of filtered groups

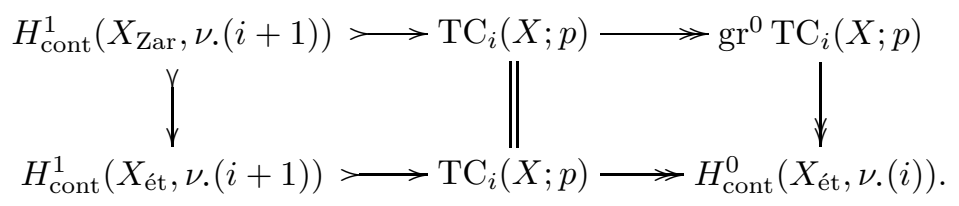

We note that the exact sequence provided by the snake lemma coincides with the exact sequence of corollary 5.4.3. 
5.5. The cyclotomic trace

$$
\operatorname{trc}: K_{*}\left(X ; \mathbb{Z}_{p}\right) \rightarrow \mathrm{TC}_{*}(X ; p)
$$

induces a map of Zariski descent spectral sequences, and according to corollary 4.2.4 the induced map of $E_{2}$-terms,

$$
H_{\text {cont }}^{2}\left(X_{\text {Zar }},\left(\mathcal{K} / p^{*}\right)(-t)\right) \rightarrow H^{s}\left(X_{\text {Zar }}, \mathcal{T C}_{-t}\right)
$$

is a section of the map $\iota$. We note, however, that the image of $s$ need not be equal to the subgroup of $d_{2}$-cycles even though the two groups are abstractly isomorphic. Hence one might well have non-trivial $d_{2}$-differentials in the descent sequence for $K$-theory. At any rate, we get a map of filtered groups

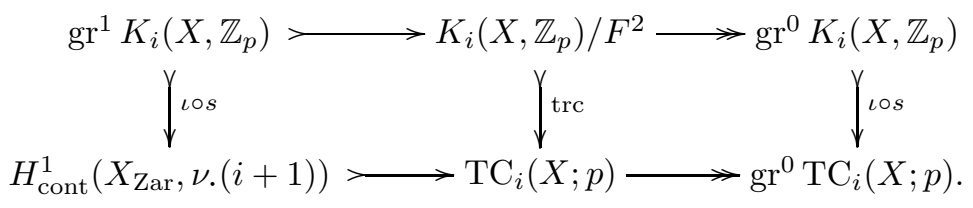

The vertical maps are all injective, so in particular, we may conclude the kernel of the cyclotomic trace is equal to the subgroup $F^{2} K_{i}\left(X, \mathbb{Z}_{p}\right)$ of elements of filtration greater than or equal to two. Hence topological cyclic homology detects only elements of weights less than or equal to $i+1$ in $K_{i}\left(X ; \mathbb{Z}_{p}\right)$. However, we do not know of an example where such elements exist.

Proposition 5.5.1. Let $X / k$ be a smooth affine scheme. Then the kernel of the cyclotomic trace

$$
\operatorname{trc}: K_{i}\left(X, \mathbb{Z}_{p}\right) \rightarrow \mathrm{TC}_{i}(X ; p)
$$

is equal to the subgroup $F^{2} K_{i}\left(X, \mathbb{Z}_{p}\right)$ of elements of filtration greater than or equal to two in the Zariski descent sequence. If $p>\operatorname{dim} X-i+1$, then the cokernel of the cyclotomic trace and of the change of topology map

$$
H_{\text {cont }}^{1}\left(X_{\text {Zar }}, \nu .(i)\right) \longmapsto H_{\text {cont }}^{1}\left(X_{\text {ét }}, \nu .(i)\right)
$$

are canonically isomorphic, and $F^{2} K_{i}\left(X, \mathbb{Z}_{p}\right)=F_{\gamma}^{i+2} K_{i}\left(X, \mathbb{Z}_{p}\right)$.

Proof. The first statement follows from the previous discussion. For the second statement, we consider Adams operations on the Zariski spectral sequence for $K$-theory. Since the $E_{2}$-terms $H_{\text {cont }}^{s}\left(X_{\text {Zar }}, \nu\right.$. $(-t)$ vanish for $-t>d$ and $s<0$, the only differentials $d_{r}$ involved in $K_{i}\left(X, \mathbb{Z}_{p}\right)$ satisfy $r \leq d-i$. But on $E_{2}^{s, t}=$ $H_{\text {cont }}^{s}\left(X_{\text {Zar }}, \nu .(-t)\right)$ the Adams operator $\psi^{k}$ acts like $k^{t}$. The usual yoga of weights then shows that for $p>d-i$, i.e. $(d-i)$ ! invertible, all differentials vanish, and all cycles are infinite cycles.

For the comparison with the $\gamma$-filtration, the argument in [10, lemma 1.1] shows that a filtration of length $d-i+1$ with given Adams operations is split and unique up to primes less than or equal to $d-i+1$. Note that we cannot hope for a better result, since the $\gamma$-filtration is only well defined up to small primes.

Example. Let $X$ be a smooth affine surface. Then the proposition shows that

$$
K_{2}\left(X, \mathbb{Z}_{p}\right) \cong \mathrm{TC}_{2}(X ; p)
$$


and yields the exact sequences

$$
\begin{aligned}
& K_{1}\left(X, \mathbb{Z}_{p}\right) \longmapsto \mathrm{TC}_{1}(X ; p) \rightarrow H_{\text {cont }}^{0}\left(X_{\text {Zar }}, R^{1} \epsilon_{*} \nu .(2)\right) \rightarrow H_{\text {cont }}^{2}\left(X_{\text {Zar }}, \nu .(2)\right) \\
& H_{\text {cont }}^{2}\left(X_{\text {Zar }}, \nu .(2)\right) \longmapsto K_{0}\left(X, \mathbb{Z}_{p}\right) \rightarrow \mathrm{TC}_{0}(X ; p) \rightarrow T_{p} \operatorname{Br}(X) .
\end{aligned}
$$

Moreover Bloch's formula $H^{j}\left(X_{\mathrm{Zar}}, \mathcal{K}_{j}\right) \cong \mathrm{CH}^{j}(X)$ and theorem 4.2.2 gives a short exact sequence

$$
{\underset{n}{\lim ^{1}}}_{p^{n}} \mathrm{CH}^{2}(X) \longmapsto H_{\text {cont }}^{2}\left(X_{\text {Zar }}, \nu .(2)\right) \rightarrow \mathrm{CH}^{2}(X)_{p}^{\wedge} .
$$

Finally,

$$
\mathrm{TC}_{-1}(X ; p) \cong H_{\text {cont }}^{1}\left(X_{\text {ét }}, \mathbb{Z} / p^{*}\right) \cong H_{\text {cont }}^{0}\left(X_{\text {Zar }}, R^{1} \epsilon_{*} \mathbb{Z} / p^{*}\right),
$$

where the last isomorphism holds since $H_{\text {cont }}^{1}\left(X_{\mathrm{Zar}}, \mathbb{Z} / p^{\cdot}\right)$ vanishes for affine $X$.

\section{Appendix: Multiplicativity of the trace}

6.1. The $K$-theory spectrum of a category $\mathcal{C}$ with cofibrations and weak equivalences as defined by Waldhausen, [42, p. 330], naturally has the structure of a symmetric spectrum. We recall the definition in a way which makes this clear.

We begin by recalling some generalities on cubical diagrams from [14]. Let $Q$ be a finite set and let $\mathcal{P}(Q)$ be the power set viewed as a category under inclusion. A $Q$-cube in $\mathcal{C}$ is a functor

$$
\mathcal{X}: \mathcal{P}(Q) \rightarrow \mathcal{C} .
$$

We call $\mathcal{X}$ a cofibration cube, if for all $S \subset T \subset Q$, the canonical map

$$
\lim _{S \subset U \varsubsetneqq T} \mathcal{X}(U) \longmapsto X(T)
$$

is a cofibration in $\mathcal{C}$. The colimit on the left may be formed by iterated pushouts along cofibrations in $\mathcal{C}$ and therefore exists.

We view the ordered set $[n]$ as a category $0 \rightarrow 1 \rightarrow \cdots \rightarrow n$. More generally, if $\mathbf{n}=\left\{n_{s}\right\}_{s \in Q}$ is a tuple of non-negative integers, we write $[\mathbf{n}]$ for the product of the categories $\left[n_{s}\right], s \in Q$. Given an arrow $\mathbf{i} \rightarrow \mathbf{j}$ in $[\mathbf{n}]$ and a subset $U \subset Q$, we define $(\mathbf{i} \rightarrow \mathbf{j})_{U}$ to be the arrow in $[\mathbf{n}]$ whose $s$ th component is $i_{s} \rightarrow j_{s}$, for $s \in U$, and $i_{s} \rightarrow i_{s}$, for $s \notin U$. Then the assignment

$$
U \mapsto(\mathbf{i} \rightarrow \mathbf{j})_{U}
$$

defines a $Q$-cube in the arrow category $\operatorname{Ar}[\mathbf{n}]$.

Let $S_{\mathbf{n}}^{Q \mathcal{C}}$ to be the full subcategory of the category of functors

$$
A: \operatorname{Ar}[\mathbf{n}] \rightarrow \mathcal{C}, \quad(\mathbf{i} \rightarrow \mathbf{j}) \mapsto A_{\mathbf{i} \rightarrow \mathbf{j}}
$$

consisting of the functors with the property that $A_{\mathbf{i} \rightarrow \mathbf{j}}=*$, if some component of $\mathbf{i} \rightarrow \mathbf{j}$ is an identity map, and that for every pair of composable arrows $\mathbf{i} \rightarrow \mathbf{j} \rightarrow \mathbf{k}$, the cube

$$
U \mapsto A_{(\mathbf{j} \rightarrow \mathbf{k})_{U} \circ(\mathbf{i} \rightarrow \mathbf{j})}
$$


is a cofibration cube and the square

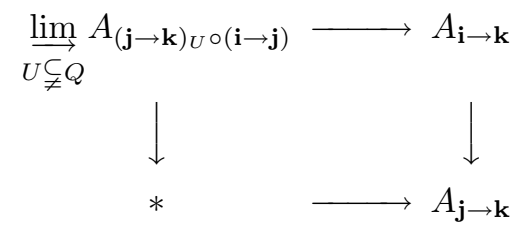

is a pushout diagram in $\mathcal{C}$. Clearly, the category $S_{\mathbf{n}}^{Q \mathcal{C}}$ depends contravariantly on [n] such that we get a $Q$-simplicial category $S .^{Q} \mathcal{C}$. Let $f: A \rightarrow A^{\prime}$ be a map in $S_{\mathbf{n}}^{Q} \mathcal{C}$. We call $f: A \rightarrow A^{\prime}$ a cofibration if for every pair of composable arrows $\mathbf{i} \rightarrow \mathbf{j} \rightarrow \mathbf{k}$, the induced map of $Q$-cubes

$$
\left(U \mapsto A_{(\mathbf{j} \rightarrow \mathbf{k})_{U} \circ(\mathbf{i} \rightarrow \mathbf{j})}\right) \rightarrow\left(U \mapsto A_{(\mathbf{j} \rightarrow \mathbf{k})_{U} \circ(\mathbf{i} \rightarrow \mathbf{j})}^{\prime}\right),
$$

viewed as a $|Q|+1$-cube in $\mathcal{C}$, is a cofibration cube. And we call $f: A \rightarrow A^{\prime}$ a weak equivalence if for every arrow $\mathbf{i} \rightarrow \mathbf{j}, f_{\mathbf{i} \rightarrow \mathbf{j}}$ is a weak equivalence in $\mathcal{C}$. With these definitions $S^{Q} \mathcal{C}$ is a $Q$-simplicial category with cofibrations and weak equivalences and a choice of ordering of $Q$ specifies an isomorphism

$$
S^{Q} \mathcal{C} \cong S . \cdots S . \mathcal{C}
$$

where the right hand side is the $|Q|$-fold iterated $S$.-construction of [42]. Let $w S{ }^{Q} \mathcal{C}$ be the subcategory of weak equivalences. Then the $n$th space of Waldhausen's $K$-theory spectrum is given by

$$
K(\mathcal{C})_{n}=\left|N . w S^{Q} \mathcal{C}\right|,
$$

where $Q=\{1, \ldots, n\}$. It is now apparent that the automorphism group of $Q$ acts on $K(\mathcal{C})_{n}$. Moreover, if $Q^{\prime}=\{1, \ldots, m\}$ then the inclusion of the 1-skeleton in the simplicial directions indexed by $Q^{\prime}$ in $S \cdot{ }^{Q^{\prime} \sqcup Q} \mathcal{C}$ defines a $\Sigma_{m} \times \Sigma_{n}$-equivariant map

$$
\lambda_{m, n}: S^{m} \wedge K(\mathcal{C})_{n} \rightarrow K(\mathcal{C})_{m+n} .
$$

With these definitions $K(\mathcal{C})$ is a symmetric spectrum. It is quasi-fibrant by [ 42 , proposition 1.5.3].

Suppose that $\mathcal{C}$ is a strict symmetric monoidal category and that the tensor product is bi-exact, that is, for every object $A$, the functors $A \otimes-$ and $-\otimes A$ preserve the null object, cofibrations and weak equivalences, and that for every pair $A \longmapsto A^{\prime}$ and $B \longmapsto B^{\prime}$ of cofibrations, the map $A^{\prime} \otimes B \oplus_{A \otimes B} A \otimes B^{\prime} \rightarrow A^{\prime} \otimes B^{\prime}$ is again a cofibration. If $Q^{\prime}$ and $Q$ are finite sets of cardinality $m$ and $n$, respectively, then the tensor product gives rise to a functor of $(m+n)$-simplicial categories with cofibrations and weak equivalences

$$
S^{Q^{\prime}} \mathcal{C} \times S_{.}^{Q} \mathcal{C} \stackrel{\otimes}{\longrightarrow} S^{Q^{\prime} \sqcup Q} \mathcal{C} ; \quad\left(A^{\prime} \otimes A\right)_{\left(\mathbf{i}^{\prime} \sqcup \mathbf{i} \rightarrow \mathbf{j}^{\prime} \sqcup \mathbf{j}\right)}=A_{\mathbf{i}^{\prime} \rightarrow \mathbf{j}^{\prime}}^{\prime} \otimes A_{\mathbf{i} \rightarrow \mathbf{j}} .
$$

Taking nerves and realization commutes with products, so we get a $\Sigma_{m} \times \Sigma_{n^{-}}$ equivariant map $K(\mathcal{C})_{m} \times K(\mathcal{C})_{n} \rightarrow K(\mathcal{C})_{m+n}$ which factors to

$$
\mu_{m, n}: K(\mathcal{C})_{m} \wedge K(\mathcal{C})_{n} \rightarrow K(\mathcal{C})_{m+n}
$$

Finally, note that $K(\mathcal{C})_{0}=|N . w \mathcal{C}|$ and let $\mathbf{1}_{0}: S^{0} \rightarrow K(\mathcal{C})_{0}$ be the map which sends the basepoint to the null object and the non-basepoint to the unit object for the tensor product. We get a $\Sigma_{n}$-equivariant map

$$
\mathbf{1}_{n}: S^{n} \rightarrow K(\mathcal{C})_{n}
$$

defined as $\mathbf{1}_{n}=\lambda_{n, 0} \circ\left(S^{n} \wedge \mathbf{1}_{0}\right)$, and with these definitions one has 
Proposition 6.1.1. ([42]) Let $\mathcal{C}$ be a category with cofibrations and weak equivalences. Then $K(\mathcal{C})$ is a quasi-fibrant symmetric spectrum. If in addition, $\mathcal{C}$ is a strict symmetric monoidal category and the tensor product is a bi-exact functor, then $K(\mathcal{C})$ is a symmetric ring spectrum.

Let $\mathcal{C}$ be an exact category in the sense of [37]. We view $\mathcal{C}$ as a category with cofibrations and weak equivalences by choosing a null object and letting cofibrations be admissible monomorphisms and weak equivalences be isomorphisms. We recall from [42, corollary 1.4.1], that in this case, the inclusion of the 0-skeleton in the nerve directions

$$
\left|\mathrm{ob} S^{Q} \mathcal{C}\right| \stackrel{\sim}{\longrightarrow}\left|N . i S^{Q} \mathcal{C}\right|=K(\mathcal{C})_{n}
$$

is a weak equivalence, provided that $Q$ is non-empty. The left hand sides again form a symmetric (ring) spectrum compatible with the inclusion maps. In the following we shall use this as our model for $K(\mathcal{C})$ for an exact category.

6.2. We now turn to topological Hochschild homology. For the purpose of studying multiplicative properties of the trace map, it is very convenient to use the model of [8], which we now recall.

Let $\mathcal{C}$ be an exact category. The set of morphisms $\operatorname{Hom}_{\mathcal{C}}\left(c, c^{\prime}\right)$ or $\mathcal{C}\left(c, c^{\prime}\right)$ is an abelian group and we define

$$
\mathcal{C}\left(c, c^{\prime}\right)_{i}=\left|\mathcal{C}\left(c, c^{\prime}\right) \otimes \mathbb{Z}\left(S^{i}\right)\right|,
$$

where we remember, $S^{i}$ is the $i$-fold smash product of the simplicial circle $\Delta^{1} / \partial \Delta^{1}$. This is an Eilenberg-MacLane space for $\mathcal{C}\left(c, c^{\prime}\right)$ concentrated in dimension $i$. The permutation of smash factors in $S^{i}$ induces a $\Sigma_{i}$-action on $\mathcal{C}\left(c, c^{\prime}\right)_{i}$, and composition in $\mathcal{C}$ defines a $\Sigma_{i} \times \Sigma_{j}$-equivariant map

$$
\mu_{i, j}^{c, c^{\prime}, c^{\prime \prime}}: \mathcal{C}\left(c^{\prime}, c\right)_{i} \wedge \mathcal{C}\left(c^{\prime \prime}, c^{\prime}\right)_{j} \rightarrow \mathcal{C}\left(c^{\prime \prime}, c\right)_{i+j}
$$

Moreover, there is a $\Sigma_{i}$-equivariant map

$$
\mathbf{1}_{i}^{c}: S^{i} \rightarrow \mathcal{C}(c, c)_{i},
$$

given by the simplicial map $x \mapsto \mathrm{id}_{c} \otimes(1 \cdot x)$. These maps satify axioms similar to those of symmetric ring spectra, see [8] for details.

Then $\operatorname{THH}(\mathcal{C} ; X)$ is defined as the realization of the cyclic space with $r$-simplices

$$
\operatorname{THH}(\mathcal{C} ; X)[r]=\underset{I^{r+1}}{\operatorname{holim}} G_{r}(\mathcal{C} ; X),
$$

where the value of the functor $G_{r}(\mathcal{C}): I^{r+1} \rightarrow \mathcal{S}_{*}$ on the object $\left(i_{0}, \ldots, i_{r}\right)$ is the pointed mapping space

$$
F\left(S^{i_{0}} \wedge \cdots \wedge S^{i_{r}}, \bigvee X \wedge \mathcal{C}\left(c_{0}, c_{r}\right)_{i_{0}} \wedge \mathcal{C}\left(c_{1}, c_{0}\right)_{i_{1}} \wedge \cdots \wedge \mathcal{C}\left(c_{r}, c_{r-1}\right)_{i_{r}}\right),
$$

where the wedge is over all $(r+1)$-tuples $\left(c_{0}, \ldots, c_{r}\right) \in$ ob $\mathcal{C}^{r+1}$ of objects in $\mathcal{C}$. The value of the functor on morphisms is defined analogous to (2). The face maps

$$
d_{j}: \operatorname{THH}(\mathcal{C} ; X)[r] \rightarrow \operatorname{THH}(\mathcal{C} ; X)[r-1] \quad, 0 \leq j \leq r,
$$

are defined in a manner similar to (4) using the natural transformations

$$
\delta_{j}: G_{r}(\mathcal{C} ; X) \rightarrow G_{r-1}(\mathcal{C} ; X) \circ \mathfrak{d}_{j}, \quad 0 \leq j \leq r,
$$


which for $0 \leq j<r$ are given by

$$
F\left(S^{i_{0}} \wedge \ldots \wedge S^{i_{r}}, \bigvee X \wedge \mathcal{C}\left(c_{0}, c_{r}\right)_{i_{0}} \wedge \ldots \wedge \mu_{i_{j}, i_{j+1}, c_{j}, c_{j+1}}^{c_{j-1}} \wedge \ldots \wedge \mathcal{C}\left(c_{r}, c_{r-1}\right)_{i_{r}}\right),
$$

and for $j=r$, by

$F\left(S^{i_{r}} \wedge S^{i_{0}} \wedge \ldots \wedge S^{i_{r-1}}, \bigvee X \wedge \mu_{i_{r}, i_{0}}^{c_{r-1}, c_{r}, c_{0}} \wedge \mathcal{C}\left(c_{1}, c_{0}\right)_{i_{1}} \wedge \ldots \wedge \mathcal{C}\left(c_{r-1}, c_{r-2}\right)_{i_{r-1}}\right) \circ \tau^{\#}$, and where as before, $\tau^{\#}=F\left(\tau^{-1}, \tau\right)$ and $\tau$ permutes the smash factors as indicated.

Denote by $G_{r}^{P}(\mathcal{C} ; X)$ the functor obtained by composing $G_{r}(\mathcal{C} ; X)$ with the concatenation functor $\sqcup_{P}: I^{P} \rightarrow I$ in each factor. We let $\operatorname{THH}^{P}(\mathcal{C} ; X)$ be the realization of the cyclic space with $r$-simplices

$$
\operatorname{THH}^{P}(\mathcal{C} ; X)[r]=\underset{\left(I^{P}\right)^{r+1}}{\operatorname{holim}} G_{r}^{P}(\mathcal{C})
$$

and with cyclic structure maps defined as above, but with the functor $\sqcup^{P}$ in place of $\sqcup$. The construction $\operatorname{THH}^{P}(\mathcal{C} ; X)$ is functorial in $X$ with respect to all continuous pointed maps and in $P$ with respect to injective maps.

Since $\mathcal{C}$ is exact, we can form the $Q$-simplicial category $S{ }^{Q} \mathcal{C}$. We let $P$ and $Q$ be finite ordered sets and consider the $S^{1}$-space

$$
\operatorname{THH}^{P}\left(S^{Q} \mathcal{C} ; X\right)=\left|[\mathbf{n}] \mapsto \operatorname{THH}^{P}\left(S_{\mathbf{n}}^{Q} \mathcal{C} ; X\right)\right|,
$$

with the $\Sigma_{P} \times \Sigma_{Q}$-action induced from the functoriality in the two factors. There is a $\Sigma_{Q^{\prime}} \times \Sigma_{Q} \times S^{1}$-equivariant map

$$
S^{Q^{\prime}} \wedge \operatorname{THH}^{P}\left(S^{Q} \mathcal{C} ; X\right) \stackrel{l}{\rightarrow} \operatorname{THH}^{P}\left(S^{Q^{\prime} \sqcup Q} \mathcal{C} ; X\right),
$$

given by the inclusion of the 1-skeleton in the simplicial directions indexed by $Q^{\prime}$ and various canonical maps, and this map is functorial in $X$ and $P$. We let $P=Q=\mathbf{n}$ and $X=S^{0}$ and define

$$
\operatorname{TH}(\mathcal{C})_{n}=\operatorname{THH}^{P}\left(S^{Q} \mathcal{C} ; S^{0}\right),
$$

which we view as a $\Sigma_{n} \times S^{1}$-space with $\Sigma_{n}$ acting via the diagonal embedding. Let

$$
\lambda_{m, n}: S^{m} \wedge \mathrm{TH}(\mathcal{C})_{n} \rightarrow \mathrm{TH}(\mathcal{C})_{m+n}
$$

be the defined as the composition of the map above and the map

$$
\mathrm{THH}^{P}\left(S^{Q^{\prime} \sqcup Q} \mathcal{C} ; S^{0}\right) \stackrel{\iota}{\rightarrow} \mathrm{THH}^{Q^{\prime} \sqcup P}\left(S^{Q^{\prime} \sqcup P} \mathcal{C} ; S^{0}\right) .
$$

One verifies that $\operatorname{TH}(\mathcal{C})$ is a symmetric spectrum with an $S^{1}$-action. DundasMcCarthy prove that if $\mathcal{C}$ is split-exact, i. e. if every admissible monomorphism in $\mathcal{C}$ has a slitting, then $\operatorname{TH}(\mathcal{C})$ is quasi-fibrant, [8, 2.1.3].

We next consider products. We first define an external product

$$
\operatorname{THH}^{P}(\mathcal{C} ; X) \wedge \mathrm{THH}^{P^{\prime}}(\mathcal{D} ; Y) \rightarrow \mathrm{THH}^{P \sqcup P^{\prime}}(\mathcal{C} \otimes \mathcal{D} ; X \wedge Y),
$$

which is $S^{1}$-equivariant, when $S^{1}$ acts diagonally on the left, and functorial with respect to inclusions in $P$ and $P^{\prime}$. Here the category $\mathcal{C} \otimes \mathcal{D}$ has as objects all pairs $(c, d)$ with $c \in$ ob $\mathcal{C}$ and $d \in$ ob $\mathcal{D}$ and

$$
(\mathcal{C} \otimes \mathcal{D})\left((c, d),\left(c^{\prime}, d^{\prime}\right)\right)=\mathcal{C}\left(c, c^{\prime}\right) \otimes \mathcal{D}\left(d, d^{\prime}\right) .
$$

The canonical map

$$
\mathcal{C}\left(c, c^{\prime}\right) \otimes \mathbb{Z}\left(S_{.}^{i}\right) \wedge \mathcal{D}\left(d, d^{\prime}\right) \otimes \mathbb{Z}\left(S^{j}\right) \rightarrow \mathcal{C}\left(c, c^{\prime}\right) \otimes \mathbb{Z}\left(S_{.}^{i}\right) \otimes \mathcal{D}\left(d, d^{\prime}\right) \otimes \mathbb{Z}\left(S^{j}\right)
$$


followed by the permutation of the middle factors and the isomorphism $\mathbb{Z}\left(S_{\text {. }}^{i}\right) \otimes$ $\mathbb{Z}\left(S^{j}\right) \cong \mathbb{Z}\left(S^{i+j}\right)$, defines a map

$$
\mathcal{C}\left(c, c^{\prime}\right)_{i} \wedge \mathcal{D}\left(d, d^{\prime}\right)_{j} \rightarrow(\mathcal{C} \otimes \mathcal{D})\left((c, d),\left(c^{\prime}, d^{\prime}\right)\right)_{i+j},
$$

and these maps, in turn, give rise to a natural transformation

$$
G_{r}^{P}(\mathcal{C} ; X) \wedge G_{r}^{P^{\prime}}(\mathcal{D} ; Y) \stackrel{\nu}{\rightarrow} G_{r}^{P \sqcup P^{\prime}}(\mathcal{C} \otimes \mathcal{D} ; X \wedge Y) \circ \text { tw },
$$

where tw is the obvious shuffle functor

$$
\left(I^{P}\right)^{r+1} \times\left(I^{P^{\prime}}\right)^{r+1} \stackrel{\mathrm{tw}}{\longrightarrow}\left(I^{P \sqcup P^{\prime}}\right)^{r+1} .
$$

One readily verifies that the induced map of homotopy colimits

$$
\begin{aligned}
\stackrel{\operatorname{holim}}{\longrightarrow} G_{r}^{P}(\mathcal{C} ; X) \wedge & G_{r}^{P^{\prime}}(\mathcal{D} ; Y) \stackrel{\nu}{\rightarrow} \underset{\stackrel{\operatorname{holim}}{\longrightarrow}}{\longrightarrow} G_{r}^{P \sqcup P^{\prime}}(\mathcal{C} \otimes \mathcal{D} ; X \wedge Y) \circ \text { tw } \\
& \stackrel{\text { can }}{\longrightarrow} \underset{\longrightarrow}{\operatorname{holim}} G_{r}^{P \sqcup P^{\prime}}(\mathcal{C} \otimes \mathcal{D} ; X \wedge Y)
\end{aligned}
$$

commutes with the cyclic structure maps. Here the left and middle homotopy colimits are indexed by the category $\left(I^{P}\right)^{r+1} \times\left(I^{P^{\prime}}\right)^{r+1}$ and the right is indexed by $\left(I^{P \sqcup P^{\prime}}\right)^{r+1}$. This gives us the desired external product map.

Suppose that $\mathcal{C}$ is strict symmetric monoidal and the tensor product bi-exact. The product (26) extends to a product

$$
S^{Q^{\prime}} \mathcal{C} \otimes S^{Q} \mathcal{C} \stackrel{\otimes}{\longrightarrow} S^{Q^{\prime} \sqcup Q} \mathcal{C}
$$

and composing this with the external product (27) we get an internal product

$$
\operatorname{THH}^{P^{\prime}}\left(S^{Q^{\prime}} \mathcal{C} ; X\right) \wedge \operatorname{THH}^{P}\left(S^{Q} \mathcal{C} ; Y\right) \rightarrow \operatorname{THH}^{P^{\prime} \sqcup P}\left(S^{Q^{\prime} \sqcup Q} \mathcal{C} ; X \wedge Y\right),
$$

which is $\Sigma_{Q^{\prime}} \times \Sigma_{Q} \times S^{1}$-equivariant and functorial in $P^{\prime}$ and $P$ and in $X$ and $Y$. This gives us a map

$$
\mu_{m, n}: \mathrm{TH}(\mathcal{C})_{m} \wedge \mathrm{TH}(\mathcal{C})_{n} \rightarrow \mathrm{TH}(\mathcal{C})_{m+n},
$$

which is $\Sigma_{m} \times \Sigma_{n} \times S^{1}$-equivariant. Finally, we note that

$$
\mathrm{TH}(\mathcal{C})_{0}=\left|N_{\wedge}^{\text {cy }}(\mathcal{C})\right|
$$

is the realization of the cyclic bar-construction of the pointed category $\mathcal{C}$. This is a cyclic set with $r$-simplices

$$
N_{\wedge}^{\mathrm{cy}}(\mathcal{C})[r]=\bigvee_{c_{0}, \ldots, c_{r} \in \mathrm{ob} \mathcal{C}} \mathcal{C}\left(c_{0}, c_{r}\right) \wedge \mathcal{C}\left(c_{1}, c_{0}\right) \wedge \ldots \wedge \mathcal{C}\left(c_{r}, c_{r-1}\right)
$$

and structure maps similar to those of $\mathrm{TH}(\mathcal{C})$. Let $e \in \mathrm{ob} \mathcal{C}$ be the identity object for the tensor product. We let $\mathbf{1}_{0}: S^{0} \rightarrow \mathrm{TH}(\mathcal{C})_{0}$ be the map which sends the non-basepoint to $\operatorname{id}_{e} \in \mathcal{C}(e, e) \subset N_{\wedge}^{\text {cy }}(\mathcal{C})[0]$ and define

$$
\mathbf{1}_{n}: S^{n} \rightarrow \mathrm{TH}(\mathcal{C})_{n}
$$

to be the $\Sigma_{n}$-equivariant map given by $\mathbf{1}_{n}=\lambda_{n, 0} \circ\left(S^{n} \wedge \mathbf{1}_{0}\right)$. One verifies that with these definitions $\operatorname{TH}(\mathcal{C})$ is a symmetric ring spectrum. Summarizing, we have

Proposition 6.2.1. If $\mathcal{C}$ is a split exact category then $\mathrm{TH}(\mathcal{C})$ is a quasi-fibrant symmetric spectrum with an $S^{1}$-action. If in addition, $\mathcal{C}$ has a strict symmetric monoidal structure such that the tensor product is bi-exact, then $\mathrm{TH}(\mathcal{C})$ is a symmetric ring spectrum. 
6.3. Finally, we are in a situation, where we can show that the trace map is multiplicative. We recall from [8] that in the model for topological Hochschild homology recalled above, the Bökstedt-Dennis trace has the following attractive description: it is the map of simplicial symmetric spectra

$$
\operatorname{tr}: K(\mathcal{C}) \rightarrow \mathrm{TH}(\mathcal{C})
$$

which on $n$th spaces in the symmetric spectra is given by the composite

$$
\left|\mathrm{ob} S_{\cdot}^{Q} \mathcal{C}\right| \rightarrow\left|N_{\wedge}^{\mathrm{cy}}\left(S^{Q} \mathcal{C}\right)[0]\right| \rightarrow\left|N_{\wedge}^{\mathrm{cy}}\left(S^{Q} \mathcal{C}\right)\right|=\mathrm{THH}^{0}\left(S^{Q} \mathcal{C}\right) \stackrel{\iota}{\rightarrow} \mathrm{THH}^{Q}\left(S^{Q} \mathcal{C}\right) .
$$

Here the first map sends an object $c \in$ ob $\mathcal{C}$ to the identity morphism $\operatorname{id}_{c} \in \mathcal{C}(c, c)$, and the second map is the inclusion of the 0 -skeleton. The image of the trace map coincides with the $S^{1}$-fixed set of $\mathrm{THH}^{Q}\left(S^{Q} \mathcal{C}\right)$. It is the subset of $\mathrm{THH}^{Q}\left(S^{Q} \cdot \mathcal{C}\right)[0]$ of those 0 -simplices for which $t_{1} s_{0} x=s_{0} x$. It is clear from the definitions that:

Proposition 6.3.1. Let $\mathcal{C}$ be an exact category. Then the Bökstedt-Dennis trace

$$
\operatorname{tr}: K(\mathcal{C}) \rightarrow \operatorname{TH}(\mathcal{C})
$$

is a map of symmetric spectra. If in addition, $\mathcal{C}$ has a strict symmetric monoidal structure such that the tensor product is bi-exact, then this is a map of symmetric ring spectra.

The construction of $\mathrm{TR}(E ; p)$ and $\mathrm{TC}(E ; p)$ from $\mathrm{TH}(E)$ given in paragraph 2 carries over verbatim to the present situation. It gives us quasi-fibrant symmetric spectra $\operatorname{TR}(\mathcal{C} ; p)$ and $\operatorname{TC}(\mathcal{C} ; p)$, which are symmetric ring spectra if $\mathcal{C}$ has a strict symmetric monoidal structure, and moreover, one has a cofibration sequence

$$
\mathrm{TH}(\mathcal{C})_{h C_{p^{n}}} \stackrel{N}{\longrightarrow} \mathrm{TH}(\mathcal{C})^{C_{p^{n}}} \stackrel{R}{\longrightarrow} \mathrm{TH}(\mathcal{C})^{C_{p^{n-1}}} .
$$

The restriction map

$$
R_{r}: \operatorname{sd}_{r}\left(\mathrm{THH}^{0}(\mathcal{C})[-]\right)^{C_{r}} \rightarrow \mathrm{THH}^{0}(\mathcal{C})[-]
$$

is an isomorphism with inverse the diagonal map

$$
N_{\wedge}^{\mathrm{cy}}(\mathcal{C}) \stackrel{\Delta_{r}}{\longrightarrow} \operatorname{sd}_{r} N_{\wedge}^{\mathrm{cy}}(\mathcal{C})^{C_{r}} .
$$

Hence the realization of the maps

$$
\begin{aligned}
\operatorname{ob} S_{.}^{Q} \mathcal{C} & \rightarrow N_{\wedge}^{\mathrm{cy}}\left(S^{Q} \mathcal{C}\right)[0] \rightarrow N_{\wedge}^{\mathrm{cy}}\left(S^{Q} \mathcal{C}\right)[-] \stackrel{\Delta_{p^{k}}}{\longrightarrow} \operatorname{sd}_{p^{k}}\left(N_{\wedge}^{\mathrm{cy}}\left(S^{Q} \mathcal{C}\right)[-]\right)^{C_{p^{k}}} \\
& =\operatorname{sd}_{p^{k}}\left(\mathrm{THH}^{0}\left(S^{Q} \mathcal{C} ; S^{0}\right)[-]\right)^{C_{p^{k}}} \stackrel{\iota}{\rightarrow} \operatorname{sd}_{p^{k}}\left(\mathrm{THH}^{Q}\left(S^{Q} \mathcal{C} ; S^{0}\right)[-]\right)^{C_{p^{k}}}
\end{aligned}
$$

composed with the isomorphism (6), defines a lifting of the Bökstedt-Dennis trace to a map of symmetric spectra

$$
\operatorname{tr}^{k+1}: K(\mathcal{C}) \rightarrow \mathrm{TR}^{k+1}(\mathcal{C} ; p),
$$

and one easily checks that these maps are compatible with the restriction maps. Moreover, there is a preferred homotopy between $F \circ \operatorname{tr}^{k+1}$ and $\operatorname{tr}^{k}$, and hence one obtains a map of symmetric spectra

$$
\operatorname{trc}: K(\mathcal{C}) \rightarrow \operatorname{TC}(\mathcal{C} ; p),
$$

the cyclotomic trace. We have 
Addendum 6.3.2. If $\mathcal{C}$ is an exact category, then the cyclotomic trace is a map of symmetric spectra

$$
\operatorname{trc}: K(\mathcal{C}) \rightarrow \mathrm{TC}(\mathcal{C} ; p) .
$$

It is a map of symmetric ring spectra, if $\mathcal{C}$ has a strict symmetric monoidal structure such that the tensor product is bi-exact.

6.4. Let $A$ be a ring and let $\mathbf{P}(A)$ be the exact category of finitely generated projective left $A$-modules. We outline a proof that $\operatorname{TH}(\tilde{A})$ and $\operatorname{TH}(\mathbf{P}(A))$ are weakly equivalent quasi-fibrant symmetric spectra.

Consider the symmetric spectrum whose $n$th space is

$$
\mathrm{TH}(\mathcal{C})_{n}^{\prime}=\operatorname{THH}^{P}\left(\mathcal{C} ; S^{R}\right),
$$

with $\Sigma_{n}$ acting diagonally, and whose structure maps are

$$
\lambda_{m, n}^{\prime}: S^{m} \wedge \mathrm{TH}(\mathcal{C})_{n}^{\prime} \rightarrow \mathrm{TH}(\mathcal{C})_{m+n}^{\prime}
$$

defined in manner analogous to the maps preceeding proposition 2.2.1. When $\mathcal{C}$ has a strict monoidal structure and the tensor product is bi-exact, then the internal product (28) gives a product

$$
\mu_{m, n}^{\prime}: \operatorname{TH}(\mathcal{C})_{m}^{\prime} \wedge \operatorname{TH}(\mathcal{C})_{n}^{\prime} \rightarrow \operatorname{TH}(\mathcal{C})_{m+n}^{\prime}
$$

and this makes $\mathrm{TH}(\mathcal{C})^{\prime}$ a symmetric ring spectrum. It is quasi-fibrant by an argument similar to the one which shows that $\mathrm{TH}(\tilde{A})$ is quasi-fibrant. One can show that the quasi-fibrant symmetric (ring) spectra $\mathrm{TH}(\mathcal{C})$ and $\mathrm{TH}(\mathcal{C})^{\prime}$ are weakly equivalent by simultaneously embedding them in the symmetric bi-spectrum $\mathrm{TH}(\mathcal{C})^{\prime \prime}$ with $(m, n)$ th space

$$
\mathrm{TH}(\mathcal{C})_{m, n}^{\prime \prime}=\mathrm{THH}^{Q \sqcup R}\left(S^{Q} \mathcal{C} ; S^{R}\right) .
$$

We leave the details to the reader.

In the case of interest to us here, there is a natural weak equivalent of quasifibrant symmetric (ring) spectra

$$
\mathrm{TH}(\tilde{A}) \stackrel{\sim}{\longrightarrow} \mathrm{TH}(\mathbf{P}(A))^{\prime}
$$

which we now explicate. Let us write $\mathcal{C}$ for $\mathbf{P}(A)$ and let $c$ be the free $A$-module of rank one. The map $A \rightarrow \mathcal{C}(c, c)$ which sends $x \in A$ to the endomorphism of $c$ given by multiplication by $x$, defines a map, or in fact a homeomorphism, $\rho_{i}: \tilde{A}_{i} \rightarrow \mathcal{C}(V, V)_{i}$. Let

$$
A_{i_{0}} \wedge \cdots \wedge A_{i_{n}} \rightarrow \bigvee \mathcal{C}\left(c_{0}, c_{r}\right)_{i_{0}} \wedge \cdots \wedge \mathcal{C}\left(c_{r}, c_{r-1}\right)_{i_{r}},
$$

be the map $\rho_{i_{0}} \wedge \cdots \wedge \rho_{i_{r}}$ followed by the inclusion of the wedge summand indexed by $(c, \ldots, c)$. This gives rise to a natural transformation

$$
\beta_{r}: G_{r}(\tilde{A} ; X) \rightarrow G_{r}(\mathcal{C} ; X)
$$

of functors from $I^{r+1}$ to the category of pointed spaces. These maps are compatible with the various structure maps, such that we get the desired map of symmetric (ring) spectra. It is a weak equivalence of quasi-fibrant symmetric spectra by $[8$, 2.1.5]. Moreover, an induction argument based on the cofibration sequences 2.3.1 and (29) shows that also

$$
\mathrm{TC}(\tilde{A} ; p) \stackrel{\sim}{\longrightarrow} \mathrm{TC}(\mathbf{P}(A) ; p)
$$


is a weak equivalence of quasi-fibrant spectra. Finally, it is proved in $[8,2.1 .6]$ that the original definition of the cyclotomic trace given in [4] and the construction of Dundas-McCarthy recalled here are compatible with this equivalence. At least the induced maps of infinite loop spaces are homotopic. In particular, we have

Corollary 6.4.1. If $A$ is a commutative ring, then the cyclotomic trace

$$
\operatorname{trc}: K_{*}(A) \rightarrow \mathrm{TC}_{*}(A ; p)
$$

is a ring homomorphism.

\section{REFERENCES}

[1] S. Bloch, K. Kato, p-adic étale cohomology, Publ. Math. IHES 63 (1986), 147-164

[2] J. M. BoArdman, Conditionally convergent spectral sequences, preprint, The Johns Hopkins University, 1981

[3] M. Bökstedt, Topological Hochschild homology, preprint Bielefeld (1985)

[4] M. Bökstedt, W.-C. Hsiang, I. Madsen, The cyclotomic trace and algebraic $K$-theory of spaces, Invent. Math. 111 (1993), 465-540

[5] M. Bökstedt, I. Madsen, Topological cyclic homology of the integers, Astérisque 226 (1994), $57-143$

[6] M. Bökstedt, I. Madsen, Algebraic $K$-theory of local number fields: the unramified case, Annals of Math. Studies.

[7] A. K. Bousfield, D. M. Kan, Homotopy Limits, Completions and Localizations, LNM 304, Springer-Verlag 1972

[8] B. I. Dundas, R. McCarthy, Topological Hochschild homology of ring functors and exact categories, J. Pure Appl. Alg. 109 (1996), 231-294

[9] O. Gabber, $K$-theory of henselian local rings and hensilian pairs, Contemporary Math., 126 (1992), 59-70

[10] T. GEISSER, $p$-adic $K$-theory of Hecke characters of imaginary quadratic fields and an analogue of Beilinson's conjecture, Duke Math. J. 86 (1997), 197-238

[11] T. Geisser, M. Levine, The $p$-part of $K$-theory of fields of characteristic $p$, Preprint 1997

[12] T. Geisser, The $p$-part of $K$-theory in characteristic $p$, Preprint 1997

[13] S. Geller, C. A. Weibel, Étale descent for Hochschild and cyclic homology, Comment. Math. Helv. 66 (1991), 368-388

[14] T. Goodwillie, Calculus II: Analytic Functors, K-theory 5 (1992), 295-332

[15] M.Greenberg, Lecture on forms in many variables, Benjamin, New York (1969)

[16] M. Gros, N. Suwa, Application d'Abel-Jacobi $p$-adique et cycles algébriques, Duke Math. J. 57 (1988), 579-613

[17] M. Gros, N.SuwA, La conjecture de Gersten pour les faisceaux de Hodge-Witt logarithmique, Duke Math. J. 57 (1988), 615-628

[18] L. Hesselholt, On the $p$-typical curves in Quillen's K-theory, Acta Math. 177 (1997), 1-53

[19] L. Hesselholt, Stable topological cyclic homology is topological Hochschild homology, Asterisque 226 (1994), 174-192

[20] L. Hesselholt, I. Madsen, On the $K$-theory of finite algebras over Witt vectors of perfect fields, Topology 36 (1997), 29-102

[21] L. Hesselholt, I. Madsen, Cyclic polytopes and the $K$-theory of truncated polynomial algebras, Inv. Math. (to appear).

[22] M. Hovey, B. Shipley, J. Smith, Symmetric spectra, preprint 1997

[23] L. Illusie, Complexe de de Rham-Witt et cohomologie cristalline, Ann. Sci. ENS 12 (1979), 501-661

[24] O. Izhboldin, On $p$-torsion in $K_{*}^{M}$ for fields in characteristic $p$, Algebraic $K$-theory, Adv. Soviet Math 4(1990), 129-144

[25] U. Jannsen, Continuous étale cohomology, Math.Ann. 280 (1988), 207-245

[26] B. KAhn, $K_{2}$ d'un corps local à corps résiduel parfait, C.R. Acad. Sc. Paris Ser. I Math. 296 (1983) no. 1, 1-3

[27] D. M. KAN, On c. s. s. complexes, Amer. J. Math. 79 (1957), 449-476 
[28] K. Kato, T. Kuzumaki, The dimension of fields and algebraic $K$-theory, J. Number Theory 24 (1986) 229-244

[29] S. MacLane, Homology, Grundlehren der mathematischen Wissenschaften 114, SpringerVerlag

[30] S. MacLane, Categories for the working mathematician, GTM, Springer-Verlag

[31] J. S. Milne, Étale cohomology, Princeton Univ. Press 1980

[32] J. S. Milne, Values of zeta functions of varieties over finite fields, Amer. J. Math. 108 (1986), 297-360

[33] J. S. Milne, Motivic cohomology and values of zeta functions, Compositio Math. 68 (1988), 59-102

[34] S. A. Mitchell, Hypercohomology spectra and Thomason's descent theorem, preprint 1996

[35] T. Moser, Ein Dualitätssatz für étale $p$-Torsionsgarben auf vollständigen Varietäten über einem endlichen Körper, Ph.D. Thesis, Regensburg 1996

[36] I. A. PAnin, The Hurewicz theorem and $K$-theory of complete discrete valuation rings, Math. USSR-Izv. 29 (1987), no. 1, 119-131

[37] D. Quillen, Higher algebraic K-theory: I, LNM 341, Springer-Verlag 1973, 85-147

[38] J.-P. Serre, Local fields, Graduate Texts in Mathematics 67, Springer-Verlag.

[39] A. A. Susuin, Torsion in $K_{2}$ of fields, $K$-theory 1 (1987), 5-29

[40] R. Thomason, Algebraic $K$-theory and étale cohomology, Ann.Sci.ENS 18 (1985), 437-552

[41] R. Thomason, T. Trobaugh, Higher algebraic K-theory of schemes and derived categories, Grothendieck Festschrift, Progr.Math 88 (1990)

[42] F. Waldhausen, Algebraic K-theory of spaces, LNM 1126 (1985), 318-419

Institute for Experimental Mathematics

Ellenrstr.29

45326 Essen

Massachusetts Institute of Technology

Germany

Massachusetts, MA 02139

Princeton University

Princeton

New Yersey, NJ 08544

E-mail address: geisser@exp-math.uni-essen.de, lh@math.princeton.edu 